\title{
SURFACE WATER SUPPLY \\ OF EASTERN AND CENTRAL NORTH CAROLINA
}

\author{
Edward B. Rice \\ District Engineer
}

\begin{abstract}
Prepared in cooperation with
Division of Water Resources, Inlets and Coastal Waterways, North Carolina Department of Conservation and Development
\end{abstract}

$$
\text { Open-File report } \quad \# 57-96
$$




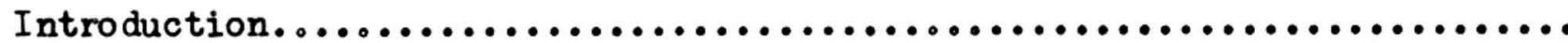

Purpose and scope..........................................

Acknowledgments............................................

Definition of terms, $\ldots \ldots \ldots \ldots \ldots \ldots \ldots \ldots \ldots \ldots \ldots \ldots \ldots \ldots \ldots \ldots \ldots \ldots \ldots \ldots \ldots \ldots \ldots \ldots \ldots \ldots \ldots \ldots$

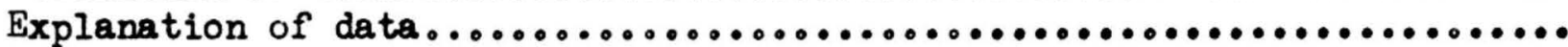
Quantity of water available..................................... Gaging-station data............................................ Chowan River basin:

Chowan River:

Wiccacon Creok:

Ahoskie Creek at Ahoskie................................

Roanoke River basin:

Roanoke River:

Dar River near Francisco $\ldots \ldots \ldots \ldots \ldots \ldots \ldots \ldots \ldots \ldots \ldots \ldots \ldots \ldots \ldots \ldots \ldots \ldots \ldots \ldots \ldots \ldots \ldots \ldots \ldots$

Mayo River near Price.

Dan River near Wentworth......................................

Smith River at Spray $\ldots \ldots \ldots \ldots \ldots \ldots \ldots \ldots \ldots \ldots \ldots \ldots \ldots \ldots \ldots \ldots \ldots \ldots \ldots \ldots \ldots \ldots \ldots \ldots \ldots \ldots$ Roanoke River at Roanoke Rapids $\ldots \ldots \ldots \ldots \ldots \ldots \ldots \ldots \ldots \ldots \ldots \ldots \ldots \ldots \ldots \ldots \ldots \ldots \ldots \ldots \ldots \ldots$ Roanoke River near Scotland Neck ................................ Pamlico River basin:

Tar River (head of Pamlico River) near Tar River.................... Tar River near Nashville.......................................

Sapony Creek near Nashville.................................. Fishing Creek near Enfield.................................

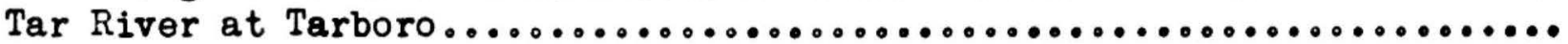
Runyon Creek:

Herring Run near Washington.............................. Neuse River basin:

Eno River (head of Neuse River) at Hillsboro....................... Flat River at Bahama $\ldots \ldots \ldots \ldots \ldots \ldots \ldots \ldots \ldots \ldots \ldots \ldots \ldots \ldots \ldots \ldots \ldots \ldots \ldots \ldots \ldots \ldots \ldots \ldots \ldots \ldots \ldots \ldots$

Dial Creek near Bahama..................................... Neuse River near Northside....................................... Neuse River near Clayton...................................... Swift Cre日k:

Middle Creek near Clayton................................ Little River near Princeton...................................

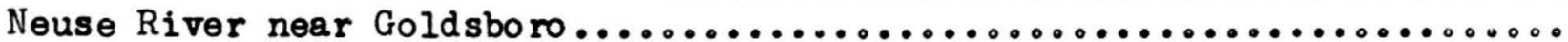
Neuse River at Kinston....................................... Contentnea Creek:

Nahunta Swamp near Shine................................. Contentnea Creek at Hookerton............................... Swift Creek near Vanceboro ..................................... Trent River near Trenton..................................... New River basin:

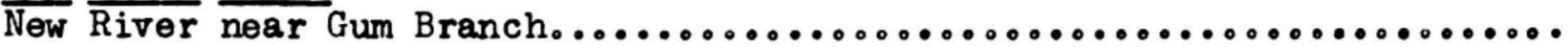


Cape Fear River basin:

Haw River (head of Cape Fear River) near Benaja....................... Reedy Fork:

Horsepen Creek at Battle Ground.................................

Reedy Fork near Gibsonville.....................................

South Buffalo Creek (head of Buffalo Creek) near Greensboro............

North Buffalo Creok near Greensboro.............................

Stony Creek near Burlington..................................... Haw River at Haw River......................................... Haw River near Pittsboro.........................................

New Hope River near Pittsboro.....................................

West Fork Deep River near High Point................................

East Fork Doep River near High Point............................

Deep River near Randleman........................................

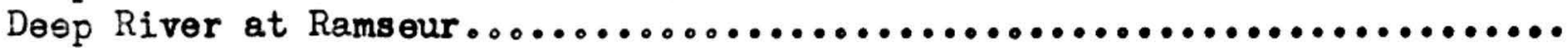

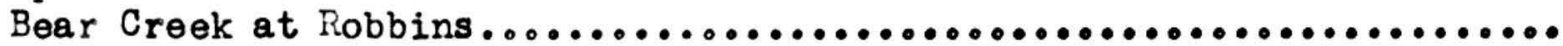

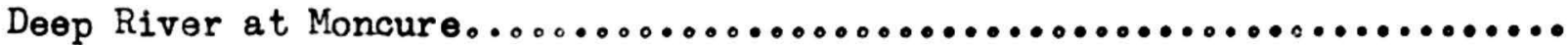
Cape Fear River at Lillington...................................

Littie River at Linden... Cape Fear River at lock 3, near Tarheel $\ldots \ldots \ldots \ldots \ldots \ldots \ldots \ldots \ldots \ldots \ldots \ldots \ldots \ldots \ldots \ldots \ldots \ldots \ldots$ Black River:

Coharie Creek:

Little Coharie Creek near Roseboro.............................

Black River near Tomahawk..................................... South River near Parkersburg................................. Colly Creek near Kelly....................................

Northeast Cape Fear River near Chinquapin....................... Waccamaw River basin:

Waccamaw River at Freeland..................................... Yadkin-Pee Dee River basin:

Yadkin River (head of Pee Dee River) at Patterson.....................

Reddies River at North Wilkesboro $\ldots \ldots \ldots \ldots \ldots \ldots \ldots \ldots \ldots \ldots \ldots \ldots \ldots \ldots \ldots$ Yadkin River at Wilkesboro....................................

Fisher River near Copeland.......................................

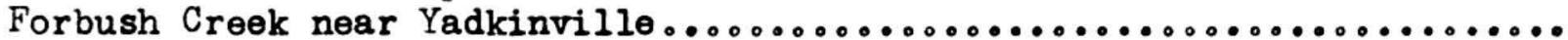
Yadkin River at Yadkin College............................. South Yadkin River:

Rocky River at Turnersburg.................................

South Yadkin River near Mocksville............................

Hunting Creek near Harmony $\ldots \ldots \ldots \ldots .0 . \ldots \ldots \ldots \ldots \ldots \ldots \ldots \ldots \ldots \ldots \ldots \ldots \ldots \ldots$

South Yadkin River at Cooleeme日,0...............................

Third Creek at Cleveland..................................

A.bbotts Creek at Lexington.....................................

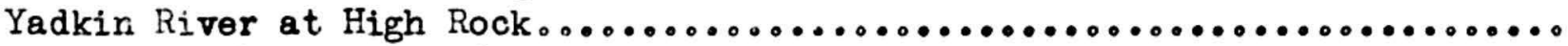
Uwharrie River near Eldorado.................................... Rocky River:

Long Creek:

Big Bear Creek near Richfield.............................. 
Yadkin-Pee Dee River basin-Continued.

Rocky River near Norwood.......................................

Brown Creok near Polkton........................................

Little River near Star .............................................

Pee Dee River near Rockingham................................... Little Pee Dee River:

Drowning Creek (head of Lumber River) near Hof fman...................

Lumber River at Boardman $\ldots \ldots \ldots \ldots \ldots \ldots \ldots \ldots \ldots \ldots \ldots \ldots \ldots \ldots \ldots \ldots \ldots \ldots \ldots \ldots \ldots \ldots \ldots \ldots$ Catauba River basin:

Catawba River (hes 1 of Santee River) near Marion........................

Linville River at Branch..........................................

Lower Little River near All Healing Springs..........................

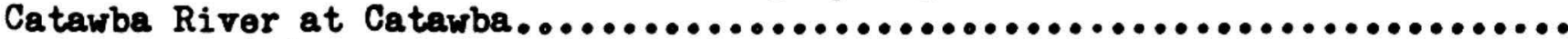

Henry Fork (head of South Fork Catawbe River) near Henry River..........

Indian Creek near Laboratory ..................................... South Fork Catawba River:

Long Creek near Bessemer City................................... South Fork Catawba River at Lowell................................. Sugar Creek:

Little Sugar Creek near Charlotte................................. Broad River basin:

Broad River near Chimney Rock.....................................

Cove Creek near Lake Lure.......................................

Second Broad River at Cliffside...................................

Broad River near Boiling Springs...................................

First Broad River near Lawndale. New (Kanawha) River basin:

South Fork New River near Jefferson................................

North Fork New River near Crumpler.............................. 


\section{INTRODUCTION}

None of the many factors that influence the economic growth of eastern and central North Carolina plays a more important, role than water. Adequate water supply for municipal, rural domestic, industrial, and agricultural uses, is a basic need of any region.

\section{Purpose and Scope}

This report summarizes part of the information on surface waters obtained at stream-gaging stations in the general area of the Piedmont and Coastal Plain of North Carolina. This information will be useful in planning the development, control, and utilization of surface water.

The present and potential users of surface waters require ample supplies of suitable quality. The increases in population, number of water-using household appliances, irrigation, and industrial activity have caused an upsurge in water requirements. Information on the availability of surface waters is essential in appraising the supplies and their adequacy to meet requirements.

This report presents streamflow information for an area of North Carolina east of the Blue Ridge Mountains and that of New River, which is the headwaters of the Kanawha River. This area is in the Coastal Plain, the Piedmont province, and a part of the Blue Ridge province.

Information is presented herein for 88 of the stream-gaging stations that the Geological Survey was operating in the area as of September 30, 1955. Records began as early as 1896 on Roanoke River at Neal, Tar River at Tarboro, and Catawba River at Catawba. Since that time, many additional stream-gaging stations have been added.

\section{Acknow ledgments}

This report was prepared in the Raleigh district office of the Surface Water Branch, Water Resources Division, U. S. Geological Survey, as part of the cooperative program with the North Carolina Department of Conservation and Development. The Nater Resources Division of the Geological Survey is under the direction of Luna B. Loopold, Chief Hydraulic Engineer, with J. V. B. Wells, Chief of the Surface Water Branch. Surface-water work in North Carolina is 
directed by Edward B. Rice, District Engineer. The North Carolina Department of Conservation and Development is under the administration of W. P. Saunders, Director, with B。C。Snow, Chief Engineer of Division of Water Resources, Inlets and Coastal Waterways.

The streamflow data sumarized in this report were collected by the U. S. Geological Survey in cooperation with the North Carolina Department of Conservation and Development, the Corps of Engineers, Department of the Army, and the cities of Burlington, Durham and Gastonia.

\section{Definition of Terms}

The streamf'low terms used in this report are defined as follows:

Cubic foot per second (cfs) is the rate of discharge of a stream whose channel is 1 square foot in cross-sectional area and whose average velocity is 1 foot per second.

Million gallons is used to express an actual quantity of water. As an absolute quantity, it is used in the measurement of storage capacities of reservoirs.

Million gallons a day (mgd) indicates a rate of $\mathrm{flow}, 1,000,000$ gallons being aten as the unit of quantity and 24 hours as the unit of time.

Convenient equivalents for the above rates of discharge are:

$$
\begin{aligned}
& 1 \mathrm{cfs}=0.646 \mathrm{mgd} \\
& 1 \mathrm{mgd}=1.55 \mathrm{cfs}
\end{aligned}
$$

In the table of contents, gaging-station records are listed in downstream order. Each indention in the listing represents one rank in the system of tributaries to the main stem. This downstream order and method of indention show which gaging stations are on tributaries entering between any two stations on a main stem, and the rank of the tributary on which each gaging station is situated.

\section{Explanation of Data}

A stream-gaging station is a site where a record of stage (gage height) of the stream is obtained, measurements of flow are made, and for which daily discharge is computed. Records of stage at stream-gaging stations are obtained from a water-stage recorder, which produces a continuous record, or from direct readings of a nonrecording gage. Measurements of flow at stream-gaging stations are made with a current meter by methods described in Water-Supply Paper 888 of the Geological Survey. Records of daily discharge at stream-gaging stations are published annually by water years (October 1 to September 30) as WaterSupply Papers of the Geological Survey in the series entitled "Surface Water 
Supply of the United States."

The data presented for each gaging station in this report generally consist of a description of the station, a flow summary, and remarks. The description of each station gives the location, gage datum, drainage area, and records available. The "Records available" paragraph gives the periods for which records of the site, or records equivalent to those at the present site have been published.

The flow summary presents date for the period that records are available, except in those instances where records are fragmentary or are otherwise not suitable for this report. Rate of flow is expressed in terms generally used in local practices. For example, flood flows are expressed in cubic feet per second whereas municipal water requirements are generally given in million gallons a day.

The "Remarks" paragraph for each gaging station gives information on conditions that affect the natural flow at the gaging station.

\section{QUANTI TY OF WATER AVAILABLE}

Average discharge is one of the most important single indications of available flow in that it represents the upper limit of struamflow development. In the flow summary for each station, average discharge is given for the number of years indicated. Although the la rge streams have the highest average discharge, streams adjacent to the boundary between the Piedmont and Blue Ridge provinces have the highest average discharge per square mile (more than $2.0 \mathrm{cfs}$ per square mile). The lowest average discharge per square mile (about 0.7 cfs per square mile) is found in the smaller streams near Wadesboro and some coastal areas.

Flood discharges and stages are of interest to all users of a stream and its adjacent lands. In the flow summaries, only the maximum instantaneous discharge during the period of record, and occasionally the discharge for a second outstanding flood, are show. For detailed information on floods the reader is referred to the report "Floods in North Carolina, Magnitude and Frequency" by H. C. Riggs.

The minimum flows of an unregulated stream observed during the period of record are an indication of the minimum sustained yields that may be expected under similar drought conditions in the future, provided flow is not diverted and used upstream. The table of minimum discharge presented for each gaging station shows the average discharge for the number of consecutive days or months indicated. The minimum flow for 1 day will be of interest on some streams, but the 7-day flow would be a more suitable criterion on a stream subject to slight fluctuations as might be caused by the operation of a mill. Information for longer periods of minimum flows might be required by planners when considering streams subject to greater amounts of regulation and where more storage is to be provided. 
The table showing duration of daily flows reveals the extent of the variation in runoff at each gaging station. This table shows the percentage of time during which indicated flows were equaled or exceeded during the period of record. The data presented herein were taken from flow-duration curves. For most stations the discharges tabulated in the duration table range from near the minimum flow to the average discharge. The range of discharges is an indication of the natural storage in a basin. At stations where the range in discharge is manyfold the yields are not well-sustained. At stations where the highest discharge shown is only a few times the lowest discharge, the low flows are rather well-sustained. In the area covered by this report, stations in western Piedmont and Blue Ridge provinces generally show the smaller range in flow and the better sustained base flows.

During dry periods streamflow may be inadequate to meet the minimum requirements for many uses without the aid of a storage reservoir. Storage of streamflow is often provided for municipal and industrial water supplies, power generation, irrigation, flood control, and other uses. The amount of storage that would have been required to maintain the indicated flows during the period of record as shown for each stream-gaging station was taken from draft-storage curves. The table shows the additional net storage that would have been required to maintain specific minimum outflow rates during the period of record. No allowance was made for quantities of water necessary to provide for dead storage, evaporation, and leakage. During dry periods much of the a vai lable low flow of many streams in the State, particularly of the smaller ones, is already utilized. On these streams an increase in usage at low flow cannot be obtained from the stream in its natural flow regimen. Thus, future planning of stream usage must carefully appraise the supply and provide operations to suit existing conditions, or must provide for supplementing low flows by the use of storage reservoirs. 


\section{CHOWAN RIVER BASIN \\ Ahoskie Creek at Ahoskie \\ Station No. 1}

Location.-Lat $36^{\circ} 17^{\prime}$, long. $77^{\circ} 00^{\prime}$, at bridge on State Highway 350 , half a mile upstream from Atlantic Coast Line Railroad bridge, and three-quarters of a mile southwest of Ahoskie, Hertford County. Altitude of gage is $22 \mathrm{ft}$.

Draingge area. -64.3 sq $\mathrm{mi}$.

Records available.-January 1950 to September 1955.

Flow oummary.-1950-55:

Average discharge ( 5 years), 48.7 cfs.

Maximum discharge, 1, 420 cfs Sept. 22, 1955 (gage height, $8.77 \mathrm{ft}$ ).

Flood of August 1940 reached a stage of $11.1 \mathrm{ft}$.

Minimum discharge for indicated consecutive period:

\begin{tabular}{ccc} 
Period & Discharge (cfs) & \multicolumn{1}{c}{ Dates } \\
1-day & 0 & Aug. 11, 1950 \\
7-day & 0 & Aug. 11-17, 1950 \\
10-day & 0 & Aug. 11-20, 1950 \\
20-day & 0 & Sept. 2-21, 1953 \\
30-day & .02 & June 28-July 27, 1954 \\
60-day & .03 & Sept. 6-Nov. 4, 1954 \\
90-day & .16 & Sept. 1-Nov. 29, 1954 \\
120-day & .17 & June 28-Oct. 25, 1954 \\
183-day & .28 & June 7-Dec. 6, 1954 \\
12 month & 12.4 & June 1954-May 1955
\end{tabular}

Duration of daily flow

Storage required to maintain indicated flow

\section{Percent timo}

indicated flow was Discharge

equaled or exceeded

$\begin{array}{lc}20 & 42.0 \\ 30 & 20.0 \\ 50 & 5.8 \\ 70 & .6 \\ 80 & .09 \\ 86 & .05\end{array}$

Regulated flow Storage required (mgd)

$\begin{array}{lr}0.01 & 0.2 \\ .025 & .5 \\ .05 & 1.9 \\ .10 & 6.6 \\ .30 & 33 \\ .50 & 64 \\ 1.0 & 150 \\ 3.0 & 570 \\ 5.0 & 1,000 \\ 8.0 & 1,750\end{array}$


Dan River near Francisco

Station No. 2

Location.-Lat $36^{\circ} 30^{\prime} 54^{\prime \prime}$, long. $80^{\circ} 18^{\prime} 12^{n}$, $200 \mathrm{ft}$ upstream from bridge on State Highway 704, an eight of a mile downstream from Georges Mill, 3 miles east of Francisco, Stokes County, and 7.9 miles downstream from Little Dan River. Altitude of gage is $830 \mathrm{ft}$.

Drainage area.-12/4 sq $\mathrm{mi}$.

Records available. August 1924 to September 1955.

Flow summary $-1921_{4}-55$ :

Average discharge ( 31 years), 186 cfs。

Maximum discharge, 12,400 cfs Oct。19, 1937 (gage height, $12.45 \mathrm{ft}$ )。

Flood of 1916 reached a stage about $3 \mathrm{ft}$ higher than that of Oct. 19, 1937 .

Minimum discharge for indicated consecutive period:

\begin{tabular}{|c|c|c|}
\hline Period & Discharge (cfs) & Dates \\
\hline $\begin{array}{l}\text { 1-day } \\
\text { 7-day } \\
\text { 10-day } \\
20 \text {-day } \\
\text { 30-day } \\
60 \text {-day } \\
\text { 90-day } \\
120 \text {-day } \\
183 \text {-day } \\
12 \text {-month }\end{array}$ & $\begin{array}{l}30 \\
32.9 \\
34.8 \\
39.2 \\
42.6 \\
47.2 \\
50.0 \\
53.1 \\
67.1 \\
92.9\end{array}$ & 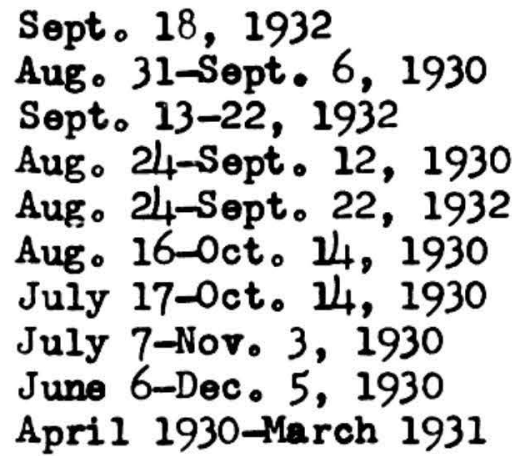 \\
\hline
\end{tabular}

Dura:ion of daily flow

Storage required to maintain indicated flow

Percent timo indicated flow was equaled or exceeded

$\begin{array}{lr}20 & 150 \\ 30 & 130 \\ 50 & 100 \\ 70 & 79 \\ 80 & 68 \\ 90 & 52 \\ 95 & 41 \\ 98 & 34 \\ 99 & 29 \\ 99.5 & 27 \\ 99.8 & 24 \\ 99.9 & 23\end{array}$

Regulated flow Storage required

\begin{tabular}{cc} 
(mgd) & (million gallons) \\
\hline 23 & 10 \\
25 & 26 \\
30 & 125 \\
35 & 350 \\
40 & 750 \\
43 & 1,000 \\
47 & 1,500 \\
50 & 2,050 \\
55 & 3,000 \\
60 & 4,100
\end{tabular}


Mayo River noar Price

Station No. 3

Location.-Lat $36^{\circ} 32^{\prime} 00^{\prime \prime}$, long. $79^{\circ} 59^{\prime} 30^{\prime \prime}, 300 \mathrm{ft}$ downstroan from Anglins Bridge, half a mile dormstream from confluence of North and South Mayo Rirers, three-quarters of a mile downstream from Virginia-North Carolim State line, and 4 miles west of Price, Rockingham County. Datum of gage is $689.95 \mathrm{ft}$ above mean sea lovel, datum of 1929 , supplementary adjustmont of 1936.

Drainage area. $-260 \mathrm{sq} \mathrm{mi}$.

Records arailable.-July 1929 to September 1955.

Flow summary. - 1929-55:

Average discharge (26 years), $321 \mathrm{cfs}$.

Maximum discharge, 30,000 cfs Oct. 19, 1937 (gage height, $4.00 \mathrm{ft}$ ).

Minimum discharge for indicated consecutive period:

$\begin{array}{cc}\text { Period } & \text { Discharge (cfs) } \\ \text { 1-day } & 35 \\ \text { 7-day } & 45.3 \\ \text { 10-day } & 47.3 \\ \text { 20-day } & 54.2 \\ 30 \text {-day } & 57.0 \\ 60 \text {-day } & 74.8 \\ 90 \text {-day } & 86.9 \\ 120 \text {-day } & 90.8 \\ 183 \text {-day } & 112 \\ 12 \text {-month } & 165\end{array}$

Dates
Oct. 8, 1954
Sept. 13-19, 1954
Sept. 10-19, 1954
Sept. 25-0ct. 14, 1954
Sept. 10-0ct. 9, 1954
Aug. 16-0ct. 14, 1954
July 18-0ct. 15, 1930
July 7-Nor. 3, 1930
June 22-Dec. 21, 1930
April 1930 to March 1931

Duration of daily flor

Storage required to maintain indicated flow

\begin{tabular}{cc}
\hline $\begin{array}{c}\text { Porcont timo } \\
\text { indicated flow was } \\
\text { equaled or exceoded }\end{array}$ & $\begin{array}{c}\text { Discharge } \\
\text { (mgd) }\end{array}$ \\
\hline 20 & 250 \\
30 & 210 \\
50 & 150 \\
70 & 120 \\
80 & 100 \\
90 & 81 \\
95 & 68 \\
98 & 56 \\
99 & 45 \\
99.5 & 38 \\
99.8 & 32 \\
99.9 & 30
\end{tabular}

\begin{tabular}{cc}
$\begin{array}{c}\text { Regulated flow } \\
\text { (mgd) }\end{array}$ & $\begin{array}{c}\text { Storage required } \\
\text { (million gallons) }\end{array}$ \\
\hline 28 & 5 \\
30 & 10 \\
40 & 130 \\
50 & 580 \\
60 & 1,200 \\
70 & 1,900 \\
80 & 2,800 \\
90 & 3,800 \\
100 & 5,500 \\
110 & 7,300
\end{tabular}

Remarke.-Slight infrequent diurnal fluctuations at low flow caused by small mills above station. 
Dan River near Wentwor th

Station No. 4

Location.-Lat $36^{\circ} 25^{\prime}$, long. $79^{\circ} 50^{\prime}$, $600 \mathrm{ft}$ downstream from Settles Bridge, 3-1/2 miles northwest of Nentworth, Rockingham County, and 7-1/2 miles downstream from Mayo River. Altitude of gage is $518 \mathrm{ft}$.

Drainge area.-1,050 sq mi. (Approximately)

Records available.-November 1939 to September 1955.

Flow surmary. -1939-55:

Average discharge (16 years), 1,161 ofs.

Maximum discharge, 56,800 cfs Sept. 18, 1945 (gage hoight, $27.78 \mathrm{ft}$ ). . :

Flood of 1908 reached a stage about $7 \mathrm{ft}$ higher than that of Sept. 18; 1945 .

Minimum discharge for indicated consecutive period:

\begin{tabular}{ccl} 
Period & Discharge (cfs) & \multicolumn{1}{c}{ Dates } \\
1-day & 107 & Oct. 2, 1954 \\
7-day & 126 & Oct. 6-12, 1954 \\
10-day & 132 & Oct. 5-14, 1954 \\
20-day & 140 & Sept. 25-0ct. 14, 1954 \\
30-day & 147 & Sept. 15-0ct. 14, 1954 \\
60-day & 230 & Aug. 16-0ct. 14, 1954 \\
90 -day & 268 & Sept. 9-Dec. 6, 1953 \\
120-day & 292 & Aug. 8-Dec. 5, 1953 \\
183-day & 394 & July 12, 1953-Jan。 10, 1954 \\
12-month & 748 & October 1953-September 1954
\end{tabular}

Duration of daily flow

Storage required to maintain indicated flow

\begin{tabular}{cc}
\hline $\begin{array}{c}\text { Percent time } \\
\text { indicated flow was } \\
\text { equaled or exceeded }\end{array}$ & $\begin{array}{c}\text { Discharge } \\
\text { (mgd) }\end{array}$ \\
\hline 20 & 890 \\
30 & 730 \\
50 & 540 \\
70 & 400 \\
80 & 340 \\
90 & 270 \\
95 & 220 \\
98 & 160 \\
99 & 136 \\
99.5 & 110 \\
99.8 & 88 \\
99.9 & 79
\end{tabular}

Regulated flow Storage required

$\begin{array}{cc}\text { (mgd) } & \text { (million gallons) } \\ 75 & 52 \\ 90 & 62\end{array}$

100

120

150

200

250

300

400

480
200

800

2,200

5,200

9,200

$\mathfrak{u}_{4}, 000$

32,000

60,000

Remarks - Slight diurnal fluctuations and regulation at low flow caused by power plants above station. 


\section{Smith River at Spray}

\section{Station No. 5}

Location. - Lat $36^{\circ} 311^{\prime} 45^{\prime \prime}$, long. $79^{\circ} 46^{\prime} 10^{n}, 0.9$ mile south of Virginia-North Carolina State line, 1 mile downstream from Stuart Creok, and 1 mile north of Spray, Rockingham County. Datum of gage is $539.55 \mathrm{ft}$ above mean sea level, datum of 1929, supplementary adjustment of 1936.

Draingge area.- -538 sq $\mathrm{mi}$.

Records available.-October 1939 to September 1955.

Flow summary.-1939-55:

Averege disoharge ( 16 years), $618 \mathrm{cfs}$, adjusted for storage since August 1950.

Maximum di scharge, 45,600 cfs Aug. 15, 1940 (gage hoight, $19.28 \mathrm{ft}$ ).

Minimum discharge for indicated consecutive period:

Period Discharge (cfs) Dates

$\begin{array}{cc}\text { 1-day } & 66.0 \\ 7 \text {-day } & 119 \\ 10 \text {-day } & 126 \\ 20 \text {-day } & 149 \\ 30 \text {-day } & 156 \\ 60 \text {-day } & 180 \\ 90 \text {-day } & 191 \\ 120 \text {-day } & 217 \\ 183 \text {-day } & 281 \\ 12 \text {-month } & 371\end{array}$

Sept. 10, 1944

Sept. 5-11, 1944

Sept. 2-11, 1944

Aug. 24-Sept. 12, 1944

Aug. 14-Sept. 12, 1944

Sept. 17-Nov. 15, 1941

Sept. 6-Dec. 4, 19/4

Aug. 7-Dec. 4, 1941

June 1-Nov. 30, 1953

October 1952-September 1953

Duration of daily flow

Storage required to maintain indicated flow

\section{Percent timo}

indicated flow was

equaled or exceeded

20

30

50

70

80

90

95

98

99

99.5

99.8

99.9
Discharge

(mgd)

460

380

290

220

190

150

130

110

94

84

70

62
Regulated flow Storage required

\begin{tabular}{cc} 
(mgd) & (million gallons) \\
\hline 45 & 2.5 \\
50 & 7.0 \\
60 & 18 \\
70 & 27 \\
80 & 40 \\
90 & 72 \\
100 & 190 \\
150 & 2,600 \\
200 & 9,000 \\
240 & 16,000
\end{tabular}

Reparke.-Flow regulated since August 1950 by Philpott Reservoir (usable capacity, 145,200 acre-feet). Some additional regulation by powerplant at Martinsvilie, Va. 


\section{Roanoke River at Roanoke Rapids}

Station No。 6

Location.-Lat $36^{\circ} 28^{\prime}$, long $77^{\circ} 38^{\prime}, 1-1 / 4$ miles downstream from bridge on State Highway 48 at Roanoke Rapids Halifax County, 2-1/2 miles upstream from Chocyott Creek, and at mile 133.6. Datum of gage is 43.84 ft above mean sea level, datum of 1929 , supplementary adjustment of 1936.

Drainage area. $-8,410 \mathrm{sq} \mathrm{mi}$.

Records available.-December 1911 to September 1955.

Flow summary. -1911-55:

Average discharge ( 44 years), 8,364 cfs, adjusted for storags since August 1950 。

Maximum discharge, 261,000 ofs Aug. 18, 1940 (gage height, $39.0 \mathrm{ft}$ ).

Flood of Norember 1877 reached a stage about $2 \mathrm{ft}$ lower than that of August 1940。

Minimum discharge for indicated consecutive period:

$\begin{array}{rr}\text { Poriod } & \text { Dischar } \\ & \\ \text { 1-day } & 472 \\ 7 \text {-day } & 521 \\ \text { 10-day } & 551 \\ \text { 20-day } & 765 \\ \text { 30-day } & 887 \\ 60 \text {-day } & 979 \\ 90 \text {-day } & 1,108 \\ \text { 120-day } & 1,305 \\ \text { 183-day } & 1,790 \\ \text { 12-month } & 3,324\end{array}$

Duration of daily flow

\begin{tabular}{|c|c|c|}
\hline $\begin{array}{c}\text { Percent time } \\
\text { indicated flow was }\end{array}$ & \multicolumn{2}{|c|}{$\begin{array}{l}\text { Discharge (mgd) } \\
1931-491950-55\end{array}$} \\
\hline 20 & 7.100 & 6,100 \\
\hline 30 & 5,500 & 4,900 \\
\hline 50 & 3,600 & 3,500 \\
\hline 70 & 2,500 & 2,600 \\
\hline 80 & 2,000 & 1,900 \\
\hline 90 & 1,500 & 1,300 \\
\hline 95 & 1,200 & 1,000 \\
\hline 98 & 870 & 840 \\
\hline 99 & 710 & 740 \\
\hline 99.5 & 550 & 600 \\
\hline 99.8 & 440 & 500 \\
\hline 99.9 & 390 & 460 \\
\hline
\end{tabular}

Datos

Sept. 21, 1932

Sopt. 19-25, 1932

Sopt。17-26, 1932

Sept.12-0ot. 1, 1932

Sept. 29-0ct. 28, 1930

Aug. 2 20ct. 27, 1930

Aug。 Oct. 30, 1930

July (-Nor. 4, 1930

June 28-Dec. 27, 1930

April 1930-March 1931

Storage required to maintein indicated flow

\begin{tabular}{cc}
\hline $\begin{array}{c}\text { Regulated flow } \\
\text { (mgd) }\end{array}$ & $\begin{array}{c}\text { Storage required } \\
\text { (million gallons) } \\
1931-49\end{array}$ \\
\hline 370 & 400 \\
390 & 600 \\
450 & 1,100 \\
560 & 2,000 \\
660 & 4,000 \\
700 & 7,000 \\
800 & 15,000 \\
1,000 & 28,000 \\
2,000 & 150,000 \\
3,000 & 420,000 \\
&
\end{tabular}

Remarks.-Flow regulated since August 1950 by Phillpot Reservoir on Smith River (usable capacity, 145,200 acre-f't) and by John H. Kerr Reservoir (usable capacity, 2,32/,000 acre-ft) and since June 1955 by Roanoke Rapids Reservoir (usable capacity 79,600 acre-ft). 
Roanoke River near Scotland Neck

Station No. 7

Location.-Lat $36^{\circ} 12^{\prime}$, I ng. $77^{\circ} 23^{\prime}$, just upstream from bridge on U. S. Highway 258 , 1 mile downstream from tributary on right, 3 miles downstream from Bricigers Creek, 5-3/4 miles north of Scotland Neck, Halifax County, and at mile 102.5. Datum of gege is $5.77 \mathrm{ft}$ above mean sea level, datum of 1929, supplementary adjustment of 1936.

Drainage area.- $-8,700 \mathrm{sq} \mathrm{mi}$.

Records available.-July 1896 to May 1903, August 1940 to September 1955.

Flow summary. $-1940-55$ :

Average discharge 1896-1902; 1940-55 (21 years) 8,862 cfs (adjusted for storage).

Maximum discharge, 260,000 cfs Aug. 19, 1940 (gage height, $41.98 \mathrm{ft}$ ).

Stage of known floods outside period of record: 1877 about $37.8 \mathrm{ft}$;

March 1912, 36.? ft; 1919, $34.9 \mathrm{ft} ; 1924,32.9 \mathrm{ft}$.

Minimum discharge for indicated consecutive period:

$\begin{array}{rr}\text { Period } & \text { Dischar } \\ \text { 1-day } & 750 \\ \text { 7-day } & 853 \\ \text { 10-day } & 969 \\ \text { 20-day } & 1,160 \\ \text { 30-day } & 1,300 \\ \text { 60-day } & 1,590 \\ 90 \text {-day } & 1,820 \\ \text { 120-day } & 2,130 \\ \text { 183-day } & 2,700 \\ \text { 12-month } & 4,770\end{array}$

Duration of daily flow

\section{Dates}

Nov. 10, 1952

Nov. 9-15, 1952

Nov. 7-16, 1952

Nov. 1-20, 1952

Oct. 22-Nov. 20, 1952

Sept. 18-Nov. 16, 1941

Sept. 10-Dec. 8, 1S-r1

Aug。 10-Dec 。 7, 1941

Aug. 10, 194l-Feb。 8, 1942

May 1941-April 1942
Percent time

indicated flow was Discharge

equaled or exceeded

20

30

50

70

80

90

95

98

99

99.5

99.8

99.9 (mgd)

7,700

5,800

3,900

2,800

2,300

1,700

1,300

1,000

970

860

740

610

Storage required to maintain indicated flow

\begin{tabular}{cc}
$\begin{array}{c}\text { Regulated flow } \\
\text { (mgd) }\end{array}$ & $\begin{array}{c}\text { Storage required } \\
\text { (million gallons) }\end{array}$ \\
\hline 540 & 56 \\
600 & 360 \\
700 & 1,100 \\
800 & 2,100 \\
1,000 & 5,800 \\
1,500 & 30,000 \\
2,000 & 78,000 \\
2,500 & 150,000 \\
3,000 & 230,000 \\
3,100 & 250,000
\end{tabular}

Remarks.-Flow regulated since August 1950 by Philpott and John H. Kerr Reservirs (combined usable capacity of 2,469,500 acre feet), and since June 1955 by Roanoke Rapids Reservoir (usable capacity, 79,600 acre-ft). 
Station No。 8

Location.-Lat $36^{\circ} 12^{\prime}$, long. $78^{\circ} 34^{\prime}, 50 \mathrm{ft}$ downstream from bridge on State Highway $96,1-1 / 4$ miles upstream from Fishing Creek, 2-1/2 miles east of town of Tar River, Granville County, and $8 \mathrm{miles}$ suth of Oxford. Datum of gage is 287.04 ft above mean sea level, datum of 1929.

Drainage area. $-161 \mathrm{sq} \mathrm{mi}$.

Records available。-November 1939 to September 1955.

Flow surmary $0--1939-55:$

Average discharge (16 years), 154 cfs。

Maximum discharge, 13,100 cfs Aug. 18, 1955 (gage hoight, $18.07 \mathrm{ft}$ )。

Minimum discharge for indicated consecutive period:

\begin{tabular}{|c|c|c|}
\hline Period & Discharge (cfa) & Datos \\
\hline $\begin{array}{l}\text { 1-day } \\
7 \text {-day } \\
10 \text {-day } \\
20-\text { day } \\
\text { 30-day } \\
60-\text { day } \\
90-\text { day } \\
120 \text {-day } \\
183 \text {-day } \\
12 \text {-month }\end{array}$ & $\begin{array}{r}0.10 \\
.15 \\
.17 \\
.23 \\
.28 \\
.38 \\
.95 \\
1.70 \\
5.24 \\
41.8\end{array}$ & $\begin{array}{l}\text { Oct. 14, 1954 } \\
\text { Oct. 8-14, 1954 } \\
\text { Oct. 5-14, 1954 } \\
\text { Nor. 4-23, 1941 } \\
\text { Nor. 1-30, 19/41 } \\
\text { Oct. 5-Dec. 3, 19/41 } \\
\text { Sept. 27-Dec. 25, 19/43 } \\
\text { Aug. 7-Dec. 4, 1953 } \\
\text { Aug. 8, 1941-Feb.6, } 1942 \\
\text { May 1941-Apr11 19/42 }\end{array}$ \\
\hline
\end{tabular}

- Duration of daily flow

Storage required to maintain indicated flow

\begin{tabular}{cc}
\hline $\begin{array}{c}\text { Percent time } \\
\text { indicated flow was } \\
\text { equaled or exceeded }\end{array}$ & $\begin{array}{c}\text { Discharge } \\
\text { (mgd) }\end{array}$ \\
\hline 20 & 102 \\
30 & 64 \\
50 & 30 \\
70 & 13 \\
80 & 7.3 \\
90 & 2.9 \\
95 & .81 \\
98 & .36 \\
99 & .24 \\
99.5 & .19 \\
99.8 & .14
\end{tabular}

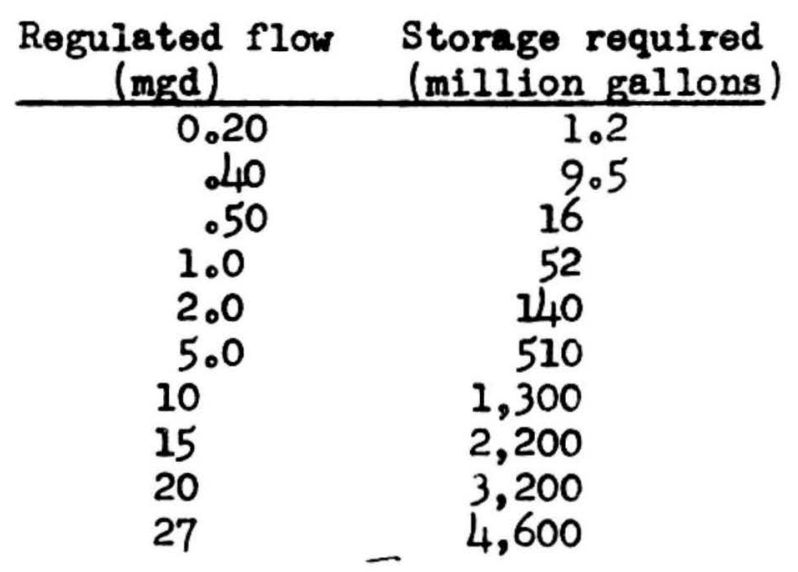

Remarks.-Recent diversions by town $f$ Oxford for municipul water supply amount to about $0.5 \mathrm{cfs}$. 
Tar River near Nashville

Station No. 9

Location.-Lat $35^{\circ} 51^{\prime} 00^{n}$, long. $77^{\circ} 55^{\prime} 50^{\prime \prime}$, downstream from Cockrell Bridge on State Highway 58, 5 miles upstream from Sapony Creek, 10 miles south of Nashville, Nash County, and at mile 93.8. Datum of gage is $110.96 \mathrm{ft}$ above mean sea lovel, datum of 1929, supplementary adjustment of 1936.

Drainage area. -701 sq $\mathrm{mi}$.

Records available.-October 1928 to September 1955.

Flow summary.-1928-55:

Average discharge (27 years), 747 cfs.

Maximum discharge, 16,900 cfs Dec。3, 1934 (gage height, $20.8 \mathrm{ft}$ )。

Minimum discharge for indicated consecutive period:

Period Discharge (cfs)

$\begin{array}{ll}\text { 1-day } & 11 \\ 7 \text {-day } & 18.7 \\ \text { 10-day } & 18.3 \\ \text { 20-day } & 22.4 \\ \text { 30-day } & 23.0 \\ 60-\text { day } & 36.4 \\ \text { 90-day } & 51.9 \\ 120 \text {-day } & 69.1 \\ 183 \text {-day } & 91.7 \\ 12 \text { - month } & 314\end{array}$

Duration of daily flow
Dates

Sept.20, 1932

Sept. 17-23, 1932

Sept. 13-22, 1932

Sept. 9-28, 1932

Aug • 30-Sept 。 28, 1932

Aug。 14-Oct。 12, 1932

July 11-Oct。 8, 1932

Sept。15, 1933-Jan. 12, 1934

Aug。 13, 1933-Feb. 11, 1934

March 1933-February 1934

Storage required to maintain indicated flow

\begin{tabular}{cc}
\hline $\begin{array}{c}\text { Percent time } \\
\text { indicated flow was } \\
\text { equaled or exceeded }\end{array}$ & $\begin{array}{c}\text { Discharge } \\
\text { (mgd) }\end{array}$ \\
\hline 20 & 600 \\
30 & 430 \\
50 & 260 \\
70 & 160 \\
80 & 120 \\
90 & 77 \\
95 & 52 \\
98 & 29 \\
99 & 19 \\
99.5 & 14 \\
99.8 & 12 \\
99.9 & 11
\end{tabular}

Regulated flow Storage required

$\frac{\text { (mgd) }}{8.0} \quad$ (million gallons)

10

15

2.0

30

50

100

3.7

150

35

150

540

2,300

10,000

20,000

200 32,000

Remarks.-Considerable diurnal fluctuation and some regulation at low flow caused by small mills upstream. 


\section{Sapony Creek near Nashville}

Station No. 10

Location.-Lat $35^{\circ} 53^{\prime} 05^{\prime \prime}$, long. $77^{\circ} 54^{\prime} 45^{\prime \prime}$, at highway bridge, 1 mile upstream from mouth ind 6-1/2 miles southeast of Nashville, Nash County. Altitude of gage $18100 \mathrm{ft}$.

Drainage area. $-64.8 \mathrm{sq} \mathrm{mi}$.

Recorde arailable. April 1950 to September 1955.

Flow summary.-1950-55:

Average discharge ( 5 years), 57.0 ofs.

Meximum discharge, 2,200 cfs Jan. 24, 1954 (gage hoight, $14.34 \mathrm{ft}$ ). Minimun discharge for indicated consecutive poriod:

$\begin{array}{cr}\text { Perlod } & \text { Discharge } \\ \text { 1-day } & 0.02 \\ 7 \text {-day } & .03 \\ 10 \text {-day } & .03 \\ 20 \text {-day } & .04 \\ 30 \text {-day } & .04 \\ 60 \text {-day } & .04 \\ 90 \text {-day } & .05 \\ 120 \text {-day } & .19 \\ 183 \text {-day } & 1.13 \\ 12 \text {-month } & 26.6\end{array}$

Dates

Sept. 3, 1954

Sopt. 2-8, 1954

Aug. 31-Sopt. 9, 1954

Aug. 31-Sept. 19, 1954

Aug. 31-Sopt. 29, 1954

Aug. 31-0ot. 29, 1954

Aug. 19-Nor. 16, 1954

July 24 -Nov。 20, 1954

June 22-Dec。21,1954

January-December 1951

Duration of daily flow

Storage required to maintain indicated $f^{7}$ ow

\begin{tabular}{cc}
\hline $\begin{array}{c}\text { Percent time } \\
\text { indicated flow was }\end{array}$ & $\begin{array}{c}\text { Discharge } \\
\text { (mgd) }\end{array}$ \\
\hline equaled or exceeded & 44 \\
20 & 29 \\
30 & 11 \\
50 & 3.1 \\
70 & 1.3 \\
80 & .24 \\
90 & .046 \\
95 & .034 \\
90 & .028 \\
99 &
\end{tabular}

Regulated flow Storage required

\begin{tabular}{cc} 
(mgd) & (million gallons) \\
\hline 0.07 & 3.2 \\
.10 & 6.0 \\
.25 & 20 \\
.50 & 46 \\
1.0 & 110 \\
2.5 & 340 \\
5.0 & 790 \\
10 & 1,800 \\
15 & 3,000 \\
17 & 3,500
\end{tabular}


Fishing Creek near Enfield

Station No. 11

Location.-Lat $36^{\circ} \mathrm{og}$, long. $77^{\circ} 42^{\prime}$, just downstream from bridge on U. S. Highway $301,2,000 \mathrm{ft}$ downstream from Atlantic Coast Line Railroad bridge, 2 miles southwest of Enfield, Halifax County, 4-3/4 miles downstream from Rocky Creek, and mile 27.7. Datum of gage is $76.26 \mathrm{ft}$ above mean sea level, datum of 1929, supplementary adjustment of 1936.

Drainage area.- $-521 \mathrm{sq} \mathrm{mi}$.

Records available. - October 1923 to September 1955.

Flow summary - $1923-55$ :

Average discharge ( 32 years), 496 cfs.

Maximum discharge, 12,600 cfs Dec。2, 1934 and Aug. 18, 19/40 (gage height, $17.72 \mathrm{ft}$ ).

Flood of Apr. 19, 1910 reached a stage of $20.1 \mathrm{ft}$ at site 2,000 ft upstream, and flood of July 24,1919 reached a stage of $19.6 \mathrm{ft}$ (discharge, 20,300 cfs),

Minimum discharge for indicated consecutive period:

$\begin{array}{cc}\text { Period } & \text { Discharge } \\ \text { 1-day } & 10 \\ \text { 7-day } & 11.4 \\ \text { 10-day } & 11.8 \\ \text { 20-day } & 12.6 \\ \text { 30-day } & 13.5 \\ \text { 60-day } & 18.2 \\ \text { 90-day } & 25.7 \\ \text { 120-day } & 32.3 \\ \text { 183-day } & 45.7 \\ \text { 12-month } & 194\end{array}$

Duration of daily flow
Dates

Sept. 21, 1954

Oct. 16-22, 1933

Oct. 16-25, 1933

Oct. 11-30, 1933

Oct. 4-Nov. 2, 1933

Sept. 22-Nov. 20, 1933

Sept. 15-Dec. 13, 1933

Sept, 15, 1933-Jan. 12, 1934

Aug。 5, 1933-Feb。 3, 1934

March 1933-February 1934

Storage required to maintain indicated flow

\begin{tabular}{cc}
\hline $\begin{array}{c}\text { Percent time } \\
\text { indicated flow was } \\
\text { equaled or exceeded }\end{array}$ & $\begin{array}{c}\text { Discharge } \\
\text { (mdg) }\end{array}$ \\
\hline 20 & 400 \\
30 & 290 \\
50 & 180 \\
70 & 110 \\
80 & 75 \\
90 & 44 \\
95 & 32 \\
98 & 19 \\
99 & 12 \\
99.5 & 10 \\
99.8 & 8.8 \\
99.9 & 8.3
\end{tabular}

Regulated flow Storage required

$\frac{(\mathrm{mgd})}{8.0}$ (million gallons)

10

33

12

100

15

230

20

30

500

50

70

100

1,300

3,900

7,600

15,000

130

25,000

Remarks.- Slight diurnal fluctuation and some regulation at low flow caused by mills upstream. 
Tar River at Tarboro

Station No. 12

Location.-Lat $35^{\circ} 53^{\prime} 40^{\prime \prime}$, long. $77^{\circ} 32^{\prime} 00^{\prime \prime}$, at bridge on U. S. Highway 64 in Tarboro, Edgecombe County, 6-1/2 miles downstream from Fishing Creek. Datum of gage is $10.37 \mathrm{ft}$ above mean sea level, datum of 1929 , supplementary adjustment of 1936.

Drainage area. $-2,140 \mathrm{sq} \mathrm{mi}$ (approximately)。

Records available. -July 1896 to December 1900, October 1931 to September 1955.

Flow summary.-1931-55:

Average discharge, 1896-1900; 1931-55 (28 years), 2,233 cfs。

Maximum discharge, 37,200 cf's Aug。 20, 1940 (gage height, $31.77 \mathrm{ft}$ )

Maximum stage known $34.0 \mathrm{ft}$, July 27, 1919 (discharge 52,800 cfs). Minimum discharge for indicated consecutive period:

Period Discharge (cfs)

$\begin{array}{cc}\text { 1-day } & 36 \\ \text { 7-day } & 40.1 \\ \text { 10-day } & 42.4 \\ \text { 20-day } & 48.9 \\ \text { 30-day } & 54.9 \\ \text { 60-day } & 80.0 \\ 90-\text { day } & 105 \\ 120 \text {-day } & 136 \\ 183 \text {-day } & 188 \\ 12 \text { month } & 914\end{array}$

Duration of daily flow

\begin{tabular}{cc}
\hline $\begin{array}{c}\text { Percent time } \\
\text { indicated flow was } \\
\text { equaled or exceeded }\end{array}$ & $\begin{array}{c}\text { Discharge } \\
\text { (mgd) }\end{array}$ \\
\hline 20 & 2,100 \\
30 & 1,400 \\
50 & 810 \\
70 & 450 \\
80 & 340 \\
90 & 210 \\
95 & 140 \\
98 & 81 \\
99 & 60 \\
99.5 & 43 \\
99.8 & 30 \\
99.9 & 26
\end{tabular}

\section{Dates}

Oct. 17, 1933

Sept. 26-oct. 2, 1932

Sept. 23-Oct. 2, 1932

Oct. 9-28, 1933

Oct. 3-Nor. 1, 1933

Sept。23-Nov。21, 1933

Sept. 18-Dec. 16, 1933

Sept。18, 1933-Jan。15, 1934

Aug. $14,1933-\mathrm{Feb} .12,1934$

July 1954-June 1955

Storage required to maintain indicated flow

Remarks.-Some diurnal fluctuations at low flow caused by mills upstream. Recent diversions for municipal water supply averaged 2.3 cfs by town of

Henderson, and 1 efs by Town of Tarboro. 
Herring Run near Washington

Station No. 13

Location.-Lat $35^{\circ} 34^{\prime} 03^{\prime \prime}$, long. $77^{\circ} 01^{\prime} 09^{\prime \prime}$, downstream from bridge on county road, one mile upstream from bridge on $U$. S. Highway $264,1-1 / 4$ miles upstream from mouth, and 2-3/4 miles northeast of Washington, Beaufort County. Altitude of gage is $2 \mathrm{ft}$.

Drainage area.-About $15 \mathrm{sq} \mathrm{mi}$.

Records available.-January 1950 to September 1955.

Flow summary.-1950-55:

Average discharge ( 5 years), 6.49 cfs.

Maximum discharge, 548 cfs Sept. 19, 1955 (gage height, $14.77 \mathrm{ft}$ ).

Flood of 1946 reached a stage of $17 \mathrm{ft}$.

Minimum discharge for indicated consecutive period:

Period Discharge (cfs) Dates

$\begin{array}{cc}\text { 1-day } & 0.7 \\ \text { 7-day } & .70 \\ \text { 10-day } & .70 \\ 20-\text { day } & .72 \\ 30-\text { day } & .73 \\ 60-\text { day } & .82 \\ 90-\text { day } & .85 \\ 120 \text {-day } & .87 \\ 183 \text {-day } & .94 \\ 12 \text { month } & 1.10\end{array}$

Nov. 18,1950

Nov. $12-18,1954$

Nov。22-Dec. 1, 1954

Nov。 12-Dec。1, 1954

Nov。 6-Dec。 5, 1954

Oct. 7-Dec。 5, 1954

Sept。7-Dec。 5, 1954

Aug。2-Dec。31, 1954

Aug。 3, 1954-Feb. 1, 1955

June 1954-May 1955

Duration of daily flow

Storage required to maintain indicated flow

\begin{tabular}{cr}
\hline $\begin{array}{c}\text { Percent time } \\
\text { indicated flow was } \\
\text { equaled or exceeded }\end{array}$ & $\begin{array}{c}\text { Discharge } \\
\text { (mgd) }\end{array}$ \\
\hline 20 & 4.20 \\
30 & 2.56 \\
50 & 1.10 \\
70 & .69 \\
80 & .59 \\
90 & .55 \\
95 & .52 \\
98 & .49
\end{tabular}

\begin{tabular}{cc}
$\begin{array}{c}\text { Regulated flow } \\
\text { (mgd) }\end{array}$ & $\begin{array}{c}\text { Storage required } \\
\text { (million gallons) }\end{array}$ \\
\hline 0.50 & 1.0 \\
.54 & 2.0 \\
.56 & 3.0 \\
.58 & 4.0 \\
.60 & 5.5 \\
.65 & 10 \\
.68 & 11 \\
.70 & 17 \\
.75 & 27 \\
.80 & 40
\end{tabular}

Remarks. - Natural runoff affected by ditches and canals above station. 
Eno River at Hillsboro

Station No。 14

Locat1on.-Lat $36^{\circ} 04^{\prime}$, long. $79^{\circ} 061,1,000 \mathrm{ft}$ downstream from bridge on State Highway 86, at Hillsboro, Orange County, and 2 miles downstream from Sevenmile Creek. Datum of gage is $487.44 \mathrm{ft}$ above mean sea level, datum of 1929, supplementary adjustment of 1936 .

Drainage area. $-66.5 \mathrm{sq} \mathrm{mi}$.

Records available. -November 1927 to September 1955 (fragmentary prior to April 1930)

Flow summary. -1928-55:

Average discharge 1930-55 (25 years), $63.7 \mathrm{cfs}$.

Maximum discherge, 11,000 cfs Sept。 18, 1945 (gage height, $20.01 \mathrm{ft}$ ). Minimum discharge for indicated consecutive period:

\begin{tabular}{|c|c|c|}
\hline Period & Discharge (cfs) & Dates \\
\hline $\begin{array}{l}\text { 1-day } \\
7-\text { day } \\
10-\text { day } \\
20 \text {-day } \\
30-\text { day } \\
60 \text {-day } \\
90 \text {-day } \\
120 \text {-day } \\
133 \text {-day } \\
12 \text { month }\end{array}$ & $\begin{array}{r}0.1 \\
.10 \\
.11 \\
.24 \\
.26 \\
.60 \\
1.06 \\
1.82 \\
2.99 \\
18.1\end{array}$ & 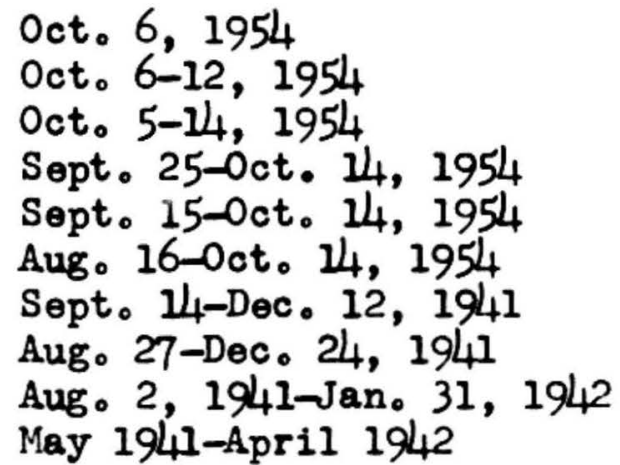 \\
\hline
\end{tabular}

Duration of daily flow

Storage required to maintain indicated flow

\begin{tabular}{cc}
\hline $\begin{array}{c}\text { Percent time } \\
\text { indicated flow was } \\
\text { equaled or exceeded }\end{array}$ & $\begin{array}{c}\text { Discharge } \\
\text { (mgd) }\end{array}$ \\
\hline 20 & 45 \\
30 & 32 \\
50 & 18 \\
70 & 10 \\
80 & 7.1 \\
90 & 3.6 \\
95 & 2.3 \\
98 & 1.1 \\
99 & .39 \\
99.5 & .24 \\
99.8 & .14 \\
99.9 & .12
\end{tabular}

Regulated flow Storage required

$\frac{(\mathrm{mgd})}{0.6} \quad$ (million gallons)

.8

1.0

2.0

3.0

5.0

7.0

8.0

10

12
43

140

260

550

920

1,100

1,600

2,000
30

Remarks.-Recent diversion of about 0.25 cfs for Hillsboro water supply is partly returned as sewage upstream. 
Flat River at Bahama

Station No。 15

Location.-Lat $36^{\circ} 11^{\prime} 00^{n}$, long. $78^{\circ} 52^{\prime} 45^{\prime \prime}$, half a mile upstream from Lake Michie, 1-1/4 miles upstream from highway bridge, 1-1/4 miles north of Bahama, Durham County, and $1-1 / 2$ miles upstream from Dial Creek. Altitude of gage is $356 \mathrm{ft}$.

Drainage area. $-150 \mathrm{sq} \mathrm{mi}$.

Records available.-July 1925 to September 1955.

Flow summary. - 1925-55:

Average discharge ( 30 years), 143 cfs.

Maximum discharge, about 20,000 cfs July 26, 1938 (gage height not determined)

Minimum discharge for indicated consecutive period:

\begin{tabular}{|c|c|c|}
\hline Period & Discharge (cfs) & Dates \\
\hline $\begin{array}{l}\text { 1-day } \\
\text { 7-day } \\
\text { 10-day } \\
\text { 20-day } \\
\text { 30-day } \\
\text { 60-day } \\
\text { 90-day } \\
120 \text {-day } \\
\text { 183-day } \\
\text { 12-month }\end{array}$ & $\begin{array}{r}0.37 \\
.42 \\
.44 \\
.57 \\
.67 \\
.82 \\
1.21 \\
1.89 \\
5.95 \\
30.6\end{array}$ & 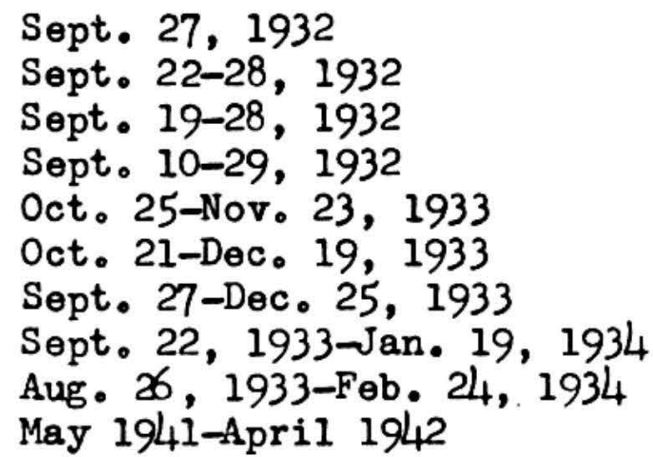 \\
\hline
\end{tabular}

Duration of daily flow

Storage required to maintain indicated flow

\section{Percent time} indicated flow was equaled or exceeded 20 30

50

70

80

90

95

98

99

99.5

99.8

99.82
Discharge (mgd)

93

61

34

16

11

4.4

2.3

.91

.56

.45

.35

.34
Regulated flow Storage required

\begin{tabular}{cc} 
(mgd) & (milliongallons) \\
\hline 1 & 27 \\
2 & 110 \\
3 & 220 \\
5 & 480 \\
7 & 800 \\
10 & 1,300 \\
13 & 1,900 \\
15 & 2,400 \\
20 & 3,500 \\
30 & 6,000
\end{tabular}

Remarks. - Some diurnal fluctuation and infrequent regulation at low flow caused by small mill 5 miles upstream. 


\section{Dial Creek near Bahama \\ Station No. 16}

Location.-Lat $36^{\circ} 10^{\prime} 35^{\prime \prime}$, long. $78^{\circ} 51^{\prime} 20^{n}$, three-eights of a mile upstream from mouth and Lake Michie, and $1-1 / 2$ miles northeast of Bahama, Durham County. Altitude of gage is $356 \mathrm{ft}$.

Drainage area. -4.9 sq $\mathrm{mi}$.

Records available. - October 1925 to September 1955.

Flow summary - $1925-55$ :

Average discharge ( 30 years), 4.31 cfs.

Maximum gage height, $7.60 \mathrm{ft}$ May 24,1940 (discharge not determined).

Minimum discharge for indicated consecutive period:

\begin{tabular}{ccc} 
Period & Discharge (cfs) & \multicolumn{1}{c}{ Dates } \\
& & \\
1-day & 0 & July 9, 1932 \\
7-day & 0 & July 9-15, 1932 \\
10-day & 0 & July 9-18, 1932 \\
20-day & 0 & July 9-28, 1932 \\
30-day & 0 & July 9-Aug. 7, 1932 \\
60-day & 0 & July 9-Sept. 6, 1932 \\
90-day & .0003 & July 7-0ct. 4, 1932 \\
120-day & .06 & June 7-0ct. 4, 1932 \\
183-day & .19 & May 26-Nov. 25, 1926 \\
12-month & 1.17 & May 1941-Apri1 1942
\end{tabular}

Duration of daily flow

Storage required to maintain indicated flow

Percent time indicated flow was equaled or exceeded

\begin{tabular}{cc} 
d or exceeded & (mgd) \\
\hline 20 & 3.1 \\
30 & 2.2 \\
50 & 1.2 \\
70 & .65 \\
80 & .37 \\
90 & .12 \\
95 & .016
\end{tabular}

\begin{tabular}{cc}
$\begin{array}{c}\text { Regulated flow } \\
\text { (mgd) }\end{array}$ & $\begin{array}{c}\text { Storage required } \\
\text { (million gallons) }\end{array}$ \\
\hline 0.02 & 1.8 \\
.05 & 4.6 \\
.10 & 9.2 \\
.20 & 19 \\
.30 & 34 \\
.40 & 50 \\
.50 & 70 \\
.60 & 88 \\
.70 & 110 \\
.80 & 130
\end{tabular}




\section{Neuse River near Northside}

Station No. 17

Location.-Lat $36^{\circ} 02^{\prime} 07^{\prime \prime}$, long. 78 $4^{\circ} 4^{\prime} 9^{\prime \prime}$, just upstream from Fish Dam bridge, $1-1 / 2$ miles downstream from Rocky Creek, and 2-1/2 miles downstream from

Seaboard Airline Railway bridge, and $2-1 / 2$ miles south of Northside, Granville County. Datum of gage $226.32 \mathrm{ft}$ above mean sea level.

Drainage area. $-526 \mathrm{sq} \mathrm{mi}$.

Records available.-July 1927 to September 1955.

Flow summary. $-1927-55$ :

Average discharge ( 28 years), 530 cfs.

Maximum discharge, 36,600 cfs Sept. 18, 1945; (maximum gage height, 31.02

ft).

Minimum discharge for indicated consecutive period:

\begin{tabular}{|c|c|c|}
\hline Period & Discharge (cfs) & Dates \\
\hline $\begin{array}{l}\text { 1-day } \\
\text { 7-day } \\
\text { 10-day } \\
\text { 20-day } \\
\text { 30-day } \\
60-\text { day } \\
\text { 90-day } \\
120 \text {-day } \\
183-\text { day } \\
\text { 12-month }\end{array}$ & $\begin{array}{l}3.1 \\
3.70 \\
3.83 \\
4.86 \\
6.82 \\
8.02 \\
9.92 \\
11.2 \\
15.6 \\
117\end{array}$ & $\begin{array}{l}\text { Sept. 20, } 1932 \\
\text { Sept. 18-24, } 1932 \\
\text { Sept. 13-22, } 1932 \\
\text { Sept. 6-25, } 1932 \\
\text { Sept. 6-Oct. 5, } 1932 \\
\text { Oct. 3-Dec. 1, } 1941 \\
\text { Sept. 7-Dec.5, } 1941 \\
\text { Aug. 8-Dec。 5, 1941 } \\
\text { Aug. 3, 1941-Feb. 1, } 1942 \\
\text { May 194l-April 1942 }\end{array}$ \\
\hline
\end{tabular}

Duration of daily flow

Storage required to maintain indicated flow

\begin{tabular}{ccccc}
\hline $\begin{array}{c}\text { Percent time } \\
\text { indicated flow was } \\
\text { equaled or exceeded }\end{array}$ & $\begin{array}{c}\text { Discharge } \\
\text { (mgd) }\end{array}$ & & $\begin{array}{c}\text { Regulated flow } \\
\text { (mgd) }\end{array}$ & $\begin{array}{c}\text { Storage required } \\
\text { (million gallons) }\end{array}$ \\
\cline { 4 - 5 } 20 & 410 & 2.6 & 1.5 \\
30 & 300 & 3.0 & 5.0 \\
50 & 140 & 5.0 & 40 \\
70 & 76 & 10 & 350 \\
80 & 49 & 20 & 1,900 \\
90 & 22 & 30 & 3,800 \\
95 & 12 & 40 & 6,000 \\
98 & 7.1 & 50 & 8,200 \\
99 & 5.4 & 65 & 11,500 \\
99.5 & 4.5 & 76 & 14,000 \\
99.8 & 3.4 & & \\
99.9 & 2.8 & &
\end{tabular}

Remarks.-Moderate diurnal fluctuation caused by power plant upstream. Flow regulated by Durham municipal dam. Of the recent diversion averaging 13 cfs for City of Durham municipal water supply, about $6-1 / 2$ cfs was returnod as sewage 3 miles upstream from Northside gage. 


\section{Neuse River near Clayton}

Station No. 18

Location.-Lat $35^{\circ} 39^{\prime}$, long. $78^{\circ} 25^{\prime}$, just downstream from bridge on State Highway 42, 1.8 miles upstream from Mill Creek and 3 miles east of Clayton, Johnston County。 Datum of gage is 128.12 feet above mean sea level.

Drainage area.--1, $140 \mathrm{sq} \mathrm{mi}$.

Records available.-July 1927 to September 1955.

Flow summary.-1927-55:

Average discharge (28 years), 1,207 cfs.

Maximum discharge, 22,900 cfs, Sept. 19, 1945 (gage height, $22.12 \mathrm{ft}$ ).

Flood of iuly 23, 1919 reached a stage of $21.15 \mathrm{ft}$ at site $1,100 \mathrm{ft}$ upstream (discharge, $21,200 \mathrm{cfs}$ )。

Minimum discharge for indicated consecutive period:

$\begin{array}{cc}\text { Period } & \text { Discharge } \\ \text { 1-day } & 45 \\ 7 \text {-day } & 52.1 \\ \text { 10-day } & 53.8 \\ \text { 20-day } & 61.6 \\ \text { 30-day } & 67.2 \\ 60-\text { day } & 92.1 \\ \text { 90-day } & 99.2 \\ 120 \text {-day } & 107 \\ 183 \text {-day } & 130 \\ \text { 12-month } & 443\end{array}$

Duration of daily flow
Dates

Sept. 11, 1932

Oct。 5-11, 1933

Oct. 6-15, 1933

Sept。2-21, 1932

Aug。29-Sept。27, 1932

Sept. 21-Nov. 19, 1933

Sept。20-Dec。18, 1933

Sept。21, 1933-Jan. 18, 1934

Aug。 27, 1933-Feb . 25, 1934

March 1933-February 1934

Storage required to maintain indicated flow

\begin{tabular}{cc}
\hline $\begin{array}{c}\text { Percent time } \\
\text { indicated flow was } \\
\text { equaled or exceeded }\end{array}$ & $\begin{array}{c}\text { Discharge } \\
\text { (mgd) }\end{array}$ \\
\hline 20 & 970 \\
30 & 680 \\
50 & 410 \\
70 & 250 \\
80 & 180 \\
90 & 120 \\
95 & 84 \\
98 & 61 \\
99 & 50 \\
99.5 & 42 \\
99.8 & 38 \\
99.9 & 35
\end{tabular}

Regulated flow Storage required

\begin{tabular}{cc} 
(mgd) & (million gallons) \\
\hline 35 & 10 \\
40 & 45
\end{tabular}

50

70

100

150

175

220

1,100

4,000

12,000

17,000

23,000

35,000

250

300

49,000 


\section{Middle Creek near Clayton}

Station No. 19

Location.-Lat $35^{\circ} 34^{\prime} 10^{\prime \prime}$, long. $78^{\circ} 35^{\prime} 30^{n}$, $300 \mathrm{ft}$ downstream from bridge on State Highway 50 , a quarter of a mile upstream from Buffalo Branch, 3-1/4 miles downstream from county line, and 9-1/4 miles southwest of Clayton, Johnston County. Altitude of gage is $177 \mathrm{ft}$.

Drainage area.- $-80.7 \mathrm{sq} \mathrm{mi}$.

Records available.-November 1939 to September 1955.

Flow summary.-1939-55:

Average discharge ( 16 years), 87.9 cfs.

Maximum discharge, 5,400 cfs Sept, 4, 1955 (gage hoight, 13.14 ft).

Minimum discharge for indicated consecutive period:

Period Discharge (cfs) Daues

$\begin{array}{cc}\text { 1-day } & 0 \\ \text { 7-day } & .06 \\ \text { 10-day } & .07 \\ \text { 20-day } & .09 \\ \text { 30-day } & .17 \\ 60-\text { day } & 1.31 \\ \text { 90- lay } & 4.21 \\ \text { 120-day } & 5.36 \\ 183-\text { day } & 8.15 \\ \text { 12-month } & 29.4\end{array}$

Oct. 11,1954

Oct. $7-13,1954$

Oct. $4-13,1954$

Sept. 25-Oct. 14, 1954

Sept. 15-Oct. 14, 1954

Aug. 16-oct. 14, 1954

Aug. 2-Nov. 24, 1951

Aug. 7-Dec. 4, 1951

June 2-Dec. 1, 1951

February 1951-January 1952

Duration of daily flow

Storage required to maintain indicated flow

\begin{tabular}{cc}
\hline $\begin{array}{c}\text { Percent time } \\
\text { indicated flow was } \\
\text { equaled or exceeded }\end{array}$ & $\begin{array}{c}\text { Discharge } \\
\text { (mgd) }\end{array}$ \\
\hline 20 & 74 \\
30 & 52 \\
50 & 28 \\
70 & 15 \\
80 & 9.0 \\
90 & 4.9 \\
95 & 2.9 \\
98 & 1.7 \\
99 & .78 \\
99.5 & .21
\end{tabular}

Regulated flow Storage required

\begin{tabular}{cc}
$(\mathrm{mgd})$ & (million gallons) \\
\hline 0.1 & 1.1 \\
.2 & 3.1 \\
.5 & 12 \\
1.0 & 33 \\
2.0 & 88 \\
5.0 & 340 \\
7.0 & 520 \\
10 & 920 \\
15 & 1,800 \\
19 & 2,800
\end{tabular}




\section{Little River near Princeton}

Station No。 20

Location.-Lat. $35^{\circ} 30^{\prime} 20^{\prime \prime}$, long. $78^{\circ} 09^{\prime} 30^{\prime \prime}$, $400 \mathrm{ft}$ downstream from highway bridge, three-quarters of a mile upstream from Little Creek, and 3 miles north of Princeton, Johnston County. Altitude of gage is $108 \mathrm{ft}$.

Dreinage area. -229 sq $\mathrm{mi}$.

Records available.-February 1930 to September 1955.

Flow summary.-1930-55:

Average discharge (25 years), 238 cfs.

Max imum discharge, 4,770 cfs Jan. 24, 1954 (gage height, $12.79 \mathrm{ft}$ ).

Flood of July 1919 reached a stage $14.57 \mathrm{ft}$; September 1924, $14.90 \mathrm{ft}$;

September 1928, $13.3 \mathrm{ft}$; October 1929, $13.47 \mathrm{ft}$.

Minimum discharge for indicated consecutive period:

\begin{tabular}{|c|c|c|}
\hline Period & Discharge (cfs) & Dates \\
\hline 1-day & 1.0 & Sept.19, 1932 \\
\hline 7-day & 1.23 & Sept. 18-24, 1932 \\
\hline 10-day & 1.30 & Sept。18-27, 1932 \\
\hline 20-day & 1.46 & Sept. 16-0ct. 5, 1932 \\
\hline 30-day & 2.22 & Sept.6-0ct。 5, 1932 \\
\hline 60-day & 5.95 & Aug. 18-0ct. 16, 1932 \\
\hline 90-day & 11.7 & Aug。 $21-N o v \cdot 18,195$ \\
\hline 120-day & 18.4 & July 27-Nov。23, 1953 \\
\hline 183-day & 30.2 & June 6-Dec。 5, 1954 \\
\hline 12-month & 75.0 & July 1954-June 1955 \\
\hline
\end{tabular}

Duration of daily flow

Storage required to maintain indicated flow

\begin{tabular}{cc}
\hline $\begin{array}{c}\text { Percent time } \\
\text { indicated flow was } \\
\text { equaled or exceer }\end{array}$ & $\begin{array}{c}\text { Discharge } \\
\text { (mgd) }\end{array}$ \\
\cline { 1 - 1 } 20 & 210 \\
30 & 140 \\
50 & 75 \\
70 & 43 \\
80 & 28 \\
90 & 17 \\
95 & 11 \\
98 & 6.1 \\
99 & 3.6 \\
99.5 & 2.3 \\
99.8 & 1.2 \\
99.9 & .87
\end{tabular}

\begin{tabular}{cc}
$\begin{array}{c}\text { Regulated flow } \\
\text { (mgd) }\end{array}$ & $\begin{array}{c}\text { Storage required } \\
\text { (million gallons) }\end{array}$ \\
\hline 1.0 & 3.6 \\
2.0 & 20 \\
3.0 & 46 \\
5.0 & 120 \\
10 & 450 \\
20 & 1,500 \\
30 & 3,100 \\
35 & 4,000 \\
40 & 5,000 \\
50 & 7,400
\end{tabular}

Remarks.-Slight diurnal fluctuation and occasionally some regulation for short periods caused by mills upstream. 


\section{Neuse River near Goldsboro}

Station No. 21

Location.-Lat $35^{\circ} 20^{\prime}$, long. $78^{\circ} 00^{\prime}$, just downstream from highway bridge, 0.2 mile upstream from Stony Creek, 1.5 miles downstream from Atlantic Coast Line Railroad bridge, 3 miles south of Goldsboro, Nayne County, 4.3 miles downstream from Little River, and at mile 135. Datum of gage $42.95 \mathrm{ft}$ above mean sea level, datum of 1929, supplementary adjustment of 1936.

Drainage area. $-2,390 \mathrm{sq} \mathrm{mi}$.

Records available.-February 1930 to September 1955.

Flow summary. -1930-55:

Average discharge (25 years), 2,415 cfs.

Maximum discharge, 30,700 cfs Sept. 23, 1945 (gage height, 25.01, at site 2.3 miles upstream.

Maximum discharge known, 38,600 cfs 0ct. 5, 1929 (gage height, $27.3 \mathrm{ft}$, at site 1.3 miles upstream

\begin{tabular}{|c|c|c|}
\hline Period & Discharge (cfs) & Dates \\
\hline $\begin{array}{l}\text { 1-day } \\
\text { 7-day }\end{array}$ & $\begin{array}{l}90 \\
97.6\end{array}$ & Sept. 14, 1932 \\
\hline 10-day & 101 & Oct. 5-14, 1954 \\
\hline 20-day & 109 & Sept. 12-Oct. 1, 1932 \\
\hline 30-day & 112 & Sept. 15-0ct. $\mathrm{I}_{4}, 1954$ \\
\hline 60-day & 158 & Aug. 18-0ct. 17, 1932 \\
\hline 90-dey & 196 & Sept. 8-Dec. 6, 1941 \\
\hline 120-day & 225 & Sept. 27, 1933-Jan. 24, 1934 \\
\hline 183-day & 384 & Aug. 29, 1933-Fob. 27, 1934 \\
\hline 12 -month & 999 & August $1941-$ July 1942 \\
\hline
\end{tabular}

Duration of daily flow

Storage required to maintain indicated flow

Percent time

indicated flow was Discharge

equaled or exceeded

20

30

50

70

80

90

95

98

99

99.5

99.8 (mgd)

2, 500

7,600

880

510

350

210

150

110

85

78

70
Regulated flow

$\frac{\text { (mgd) }}{90}$

120

150

200

300

400

450

500

600

650
Storage required (million gallons)

560

1,800

3,500

7,500

20,000

36,000

46,000

58,000

84,000

100,000 


\section{Neuse River at Kinston}

Station No。 22

Location.-Lat $35^{\circ} 15^{\prime} 30^{\prime \prime}$, long。 $77^{\circ} 35^{\prime} 10^{\prime \prime}$, at Kinston, Lenoir County, $600 \mathrm{ft}$ downstream from bridge on State Highway 11 , and at mile 90 . Datum of gage is $10.90 \mathrm{ft}$ above mean sea level, datum of 1929 , supplementary adjustment of 1936 .

\section{Drainage area.- $-2,690 \mathrm{sq} \mathrm{mi}$.}

\section{Records available.-February 1930 to September 1955.}

Flow summary $-1930-55$ :

Average discharge (25 years), 2,768 cfs 。

Maximum discharge, 25,900 cfs Sept. 27, 1945 (gage height, 22.44 ft.)

Maximum stage known, $25.0 \mathrm{ft}$, present datum, July 1919 (discharge, about $39,000 \mathrm{cfs}$ ).

Minimum discharge for indicated consecutive period:

$$
\text { Period Discharge (cfs) Dates }
$$

$\begin{array}{rr}\text { 1-day } & 126 \\ \text { 7-day } & 143 \\ \text { 10-day } & 147 \\ \text { 20-day } & 153 \\ \text { 30-day } & 160 \\ 60 \text {-day } & 227 \\ 90 \text {-day } & 260 \\ 120 \text {-day } & 285 \\ \text { 183-day } & 500 \\ \text { 12-month } & 1,110\end{array}$

Duration of daily flow

\section{Percent time}

indicated flow was Discharge

equaled or exceeded

$\begin{array}{lr}20 & 2,800 \\ 30 & 2,000 \\ 50 & 1,100 \\ 70 & 650 \\ 80 & 480 \\ 90 & 300 \\ 95 & 230 \\ 98 & 50 \\ 99 & 130 \\ 99.5 & 120 \\ 99.8 & 100\end{array}$

Sept. 26, 1932

Sept. 20-26, 1932

Sept。17-26, 1932

Sept. 8-27, 1932

Sept. 4-0ct. 3, 1932

Aug。 18-oct。 16, 1932

Oct。 7, 1933-Jan. 4, 1934

Sept. 28, 1933-Jan. 25, 1934

June 7-Dec。6, 1954

July 1954-June 1955

Storage required to maintain indicated flow

Regulated flow Storage required

$\begin{array}{cc}\text { (mgd) } & \text { (million gallons) } \\ 100 & 95 \\ 150 & 1,500\end{array}$

200

300

400

450

500

550

600

720
4,500

14,000

28,000

36,000

45,000

54,000

64,000

90,000 


\section{Nahunta Swamp near Shine}

Station No. 23

Location.--Lat $35^{\circ} 29^{\prime}$, long. $77^{\circ} 48^{\prime}$, at highway bridge 2 miles upstream from Appletree Swamp, 3-1/2 miles north of Shine, Greene County, and 8 miles northwest of Snow Hill. Altitude of gage is $48 \mathrm{ft}$.

\section{Drainage area. $-77.6 \mathrm{sq} \mathrm{mi}$.}

Records available.-July 1954 to September 1955.

Flow summary. - $1954-55$ :

Naximum discharge, 2,050 cfs Sept. 20, 1954 (gage height, $12.37 \mathrm{ft}$ ). Minimum discharge for indicated consecutive period:

\begin{tabular}{ccc} 
Period & Discharge (cfs) & \multicolumn{1}{c}{ Dates } \\
1-day & 1.0 & Oct. 7, 1954 \\
7 -day & 1.2 & Oct. 4-10, 1954 \\
10-day & 1.4 & Oct. 3-12, 1954 \\
20-day & 1.9 & Oct. 1-20, 1954 \\
30-day & 2.1 & Sept.28-0ct. 27, 1954 \\
60-day & 2.4 & Sept.2-0ct. 31, 1954 \\
90-day & 3.1 & Aug. 5-Nov.2, 1954 \\
120-day & 5.0 & July 29-Nov.25, 1954 \\
183-day & 13.3 & July 27, 1954-Jan. 25, 1955 \\
& &
\end{tabular}

\begin{tabular}{cc}
\hline $\begin{array}{c}\text { Regulated flow } \\
\text { (mgd) }\end{array}$ & $\begin{array}{c}\text { Storage required } \\
\text { (million gallons) }\end{array}$ \\
\hline 1.1 & 1.8 \\
2.0 & 27 \\
3.0 & 90 \\
4.0 & 170 \\
5.0 & 270 \\
7.0 & 500 \\
10 & 960 \\
15 & 1,800 \\
20 & 3,000 \\
30 & 6,500
\end{tabular}

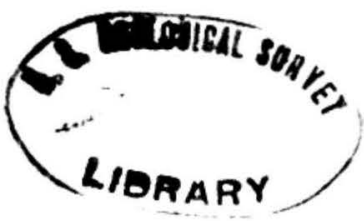


Contentnea Creek at Hookerton

Station No. 24

Location.-Lat $35^{\circ} 25^{\prime} 40^{\prime \prime}$, long $77^{\circ} 35^{\prime} 05^{\prime \prime}$, at Hookerton, Greene County, 0.3 mile upstream from bridge on State Highway 123, and 2-1/2 miles upstream from Wheat Swamp. Altitude of gage is $16 \mathrm{ft}$.

Drainge area. -729 sq mi.

Records available. -November 1928 to September 1955 .

Flow summary.-1928-55:

Average discharge, 1929-55 (26 years), 717 cfs.

Maximum discharge, 11,100 cfs 0ct. 6, 1929 (gage height, $18.9 \mathrm{ft}$ ).

Maximum stage known, $23.3 \mathrm{ft}$ in September 1928. Highwa.ter of autumn 1924 was practically same stage.

Minimum discharge for indicated consecutive period:

$\begin{array}{cc}\text { Period } & \text { Discharge } \\ \text { 1-day } & 15 \\ 7 \text {-day } & 16.4 \\ 10 \text {-day } & 16.8 \\ 20 \text {-day } & 18.2 \\ 30 \text {-day } & 19.1 \\ 60 \text {-day } & 21.2 \\ 90 \text {-day } & 26.2 \\ \text { 120-day } & 30.5 \\ 183 \text {-day } & 52.7 \\ 12 \text {-month } & 160\end{array}$

Duration of daily flow

\section{Dates}

Oct。28, 1933

Oct. $8-14,1954$

Oct. 5-14, 1954

Sept.27-0ct。16, 1954

Sept。16-0ct。15, 1954

Sept。6-Nov。 4, 1954

Aug 。 13-Nov。 10, 1954

July 28-Nov。 24, 1954

June 8-Dec. 7, 1954

June 1954-May 1955

Storage required to maintain indicated flow

Percent time

indicated flow was

equaled or exceeded

20

30

50

70

80

90

95

98

99

99.5

99.8

99.9
Discharge (mgd)

730

500

260

140

87

52

35

25

18

15

13

11
Regulated flow Storage required

$\frac{(\mathrm{mgd})}{13} \frac{\text { (mil)ion gallons) }}{32}$

$20 \quad 410$

$30 \quad 1,300$

$40 \quad 2,500$

50

70

80

90

110
3,700

6,700

7,500

10,500

15,000 


\section{Swift Creek near Vanceboro}

Station No. 25

Location.-Dat $35^{\circ} 20^{\prime} 4^{\prime \prime}$, long. $77^{\circ} 11^{\prime} 44^{\prime \prime}$, at highway bridge, 2-1/2 miles upstream from bridge on State Highway 118, 2-1/2 miles downstream from Clayroot Swamp, and 3-1/2 miles northwest of Vanceboro, Craven County. Altitude of gage is $5 \mathrm{ft}$.

Drainage area.-182 sq mi.

Records 'tvailable.-January 1950 to September 1955.

Flow simmary. -1950-55:

Average discharge ( 5 years) 124 cfs.

Maximum discharge, 6,060 cfs Sept. 22, 1955 (gage height, $13.67 \mathrm{ft}$ ).

Flood of 1909 reached a stage of $16 \mathrm{ft}$, and flood of 1928 reached a stage of $11.7 \mathrm{ft}$.

Minimum discharge for indicated consecutive period:

\begin{tabular}{ccl} 
Period & Discharge (cfs) & \multicolumn{1}{c}{ Dates } \\
1-day & 0 & Aug. 8, 1954 \\
7-day & 0 & Aug. 8-14, 1954 \\
10-day & 0 & Aug. 8-17, 1954 \\
20-day & 0 & Aug. 8-27, 1954 \\
30-day & 0 & Oct. 4-Nov. 2, 1954 \\
60-day & .20 & Sept. 28-Nov. 26, 1954 \\
90-day & .49 & Sept. 7-Dec. 5, 1954 \\
120-day & .64 & July 27-Nov. 23, 1954 \\
183-day & 1.63 & July 3, 1954-Jan. 1, 1955 \\
12-month & 8.63 & June 1954-May 1955
\end{tabular}

Duration of daily flow

Storage required to maintain indicated flow

\begin{tabular}{cc}
\hline $\begin{array}{c}\text { Percent time } \\
\text { indicated flow was } \\
\text { equaled or exceeded }\end{array}$ & $\begin{array}{c}\text { Discharge } \\
\text { (mgd) }\end{array}$ \\
\hline 20 & 81 \\
30 & 44 \\
50 & 18 \\
70 & 6.1 \\
80 & 3.6 \\
90 & 1.2 \\
95 & .30
\end{tabular}

\begin{tabular}{cc}
$\begin{array}{c}\text { Regulated flow } \\
\text { (mgd) }\end{array}$ & $\begin{array}{c}\text { Storage required } \\
\text { (million gallons) }\end{array}$ \\
\hline 0.06 & 1.8 \\
.10 & 3.6 \\
.25 & 13 \\
.50 & 34 \\
1.0 & 89 \\
2.5 & 320 \\
3.0 & 440 \\
4.0 & 620 \\
5.0 & 840 \\
6.0 & 1,100
\end{tabular}




\section{Trent River near Trenton}

Station No。 26

Location.-Lat $35^{\circ} 03^{\prime} 54^{\prime \prime}$, long. $77^{\circ} 27^{\prime} 25^{\prime \prime}, 50 \mathrm{ft}$ downstream from Free Bridge, $800 \mathrm{ft}$ downstream from Little Chinquapin Branch, 1-1/2 miles southwest of Philips Crossroads, and 6 miles west of Trenton, Jones County。 Datum of gage is $18.75 \mathrm{ft}$ above mean sea level (unadjusted).

Drainage area.- $-68 \mathrm{sq} \mathrm{mi}$.

Records available.-January 1951 to September 1955.

Flow summary $-1951-55$ :

Maximum discharge, 9,100 cfs Sept, 21, 1955 (gage height, 17.84 ft)。

Flood of 1928 reached a stage of $17.3 \mathrm{ft}$ (discharge, 7,600 cfs). Minimum discharge for indicated consecutive period:

\begin{tabular}{rcl} 
Period & Discharge (cfs) & \multicolumn{1}{c}{ Dates } \\
1-day & 1.3 & Oct. 12, 1954 \\
7-day & 1.36 & Oct。10-16, 1954 \\
10-day & 1.37 & 0ct。10-19, 1954 \\
20-day & 1.46 & Oct。9-28, 1954 \\
30-day & 1.51 & Oct。9-Nov。7, 1954 \\
60-day & 1.67 & Sept。28-Nov。27, 1954 \\
90-day & 2.31 & Sept。10-Dec。8, 1954 \\
120-day & 3.02 & Aug。11-Dec。8, 1954 \\
183-day & 4.56 & June 18-Dec。17, 1954 \\
12-month & 14.5 & June 1954-May 1955
\end{tabular}

Duration of daily flow

\begin{tabular}{cc}
\hline $\begin{array}{c}\text { Percent time } \\
\text { indicated flow was } \\
\text { equaled or exceeded }\end{array}$ & $\begin{array}{c}\text { Discharge } \\
\text { (mgd) }\end{array}$ \\
\hline 20 & 110 \\
30 & 62 \\
50 & 24 \\
70 & 7.9 \\
80 & 5.0 \\
90 & 3.2 \\
95 & 2.0 \\
98 & 1.1 \\
99 & .26 \\
99.5 & .13
\end{tabular}

Storage requirea to maintain indicated flow

\begin{tabular}{cc}
$\begin{array}{c}\text { Regulated flow } \\
\text { (mgd) }\end{array}$ & $\begin{array}{c}\text { Storage required } \\
\text { (million gallons) }\end{array}$ \\
\hline 2.0 & 56 \\
2.5 & 100 \\
3.0 & 150 \\
4.0 & 280 \\
5.0 & 420 \\
6.0 & 580 \\
7.0 & 760 \\
8.0 & 960 \\
9.0 & 1,150 \\
10 & 1,400
\end{tabular}




\section{New River near Gum Branch}

Station No. 27

Location.--Lat $34^{\circ} 51^{\prime} 05^{\prime \prime}$, long. $77^{\circ} 31^{\prime} 05^{\prime \prime}$, just downstream from highway bridge, half a mile downstream from Jenkins Swamp, 1-3/4 miles southwest of Gum Branch, Onslow County and 3-3/4 miles southwest of Richlands. Datum of gage is 3 ft above mean sea level.

Drainage area. $-74.5 \mathrm{sq} \mathrm{mi}$.

Records available.-August 1949 to September 1955.

Flow summary.-1949-55:

Average discharge (6 years), 86.8 cfs.

Maximum discharge, 7,900 cfs Sept. 20, 1955 (gage height, $19.99 \mathrm{ft}$ ).

Flood of 1908 reached a stage of about $18 \mathrm{ft}$.

Minimum discharge for indicated consecutive period:

\begin{tabular}{ccl} 
Period & Discharge (cfs) & \multicolumn{1}{c}{ Dates } \\
1-day & 1.9 & Oct. 6, 1954 \\
7-doy & 2.00 & Oct. 4-10, 1954 \\
10-day & 2.00 & Oct. 4-13, 1954 \\
20-day & 2.00 & Oct. 4-23, 1954 \\
30-day & 2.01 & Oct. 2-31, 1954 \\
60-day & 2.42 & Sept. 18-Nov. 16, 1954 \\
90-day & 3.32 & Sept. 3-Dec. 1, 1954 \\
120-day & 3.97 & Aug. 7-Dec. 4, 1954 \\
183-day & 8.79 & June 19-Dec. 18, 1954 \\
12-month & 17.4 & June 1954-May 1955
\end{tabular}

Duration of daily flow

Storage required to maintain indicated flow

Percent time

indicated flow was Discharge

equaled or exceeded.

$\begin{array}{lc}20 & 56 \\ 30 & 39 \\ 50 & 24 \\ 70 & 11 \\ 80 & 6.8 \\ 90 & 4.2 \\ 95 & 2.8 \\ 98 & 1.7\end{array}$

Regulated flow Storage required

\begin{tabular}{cc} 
(mgd) & (million gallons) \\
\hline 1.7 & 10 \\
2.0 & 30 \\
3.0 & 90 \\
4.0 & 190 \\
4.5 & 240 \\
5.0 & 300 \\
6.0 & 440 \\
8.0 & 790 \\
10 & 1,200 \\
12 & 1,600
\end{tabular}




\section{Haw River near Benaja}

Station No。 28

Location. - Lat $36^{\circ} 15^{\prime}$, long. $79^{\circ} 34^{\prime}, 200 \mathrm{ft}$ upstream from site of old High Rock Mill, $500 \mathrm{ft}$ upstream from highway bridge, half a mile upstream from county line, 6 miles downstream from Troublesome Creek, and 6 miles east of Benaje, Rockingham County. Altitude of gage is $629 \mathrm{ft}$.

Drainage area. - $168 \mathrm{sq} \mathrm{mi}$.

Records available. - October 1928 to September 1955.

Flow summary $-1928-55$ :

Average discharge (27 years) $163 \mathrm{cfs}$.

Maximum discharge, 12,300 cfs Sept. 25, 1947 (gage height, $19.22 \mathrm{ft}$ ).

Flood of July 1916 reached a stage of about $17.5 \mathrm{ft}$.

Minimum discharge for indicated consecutive period

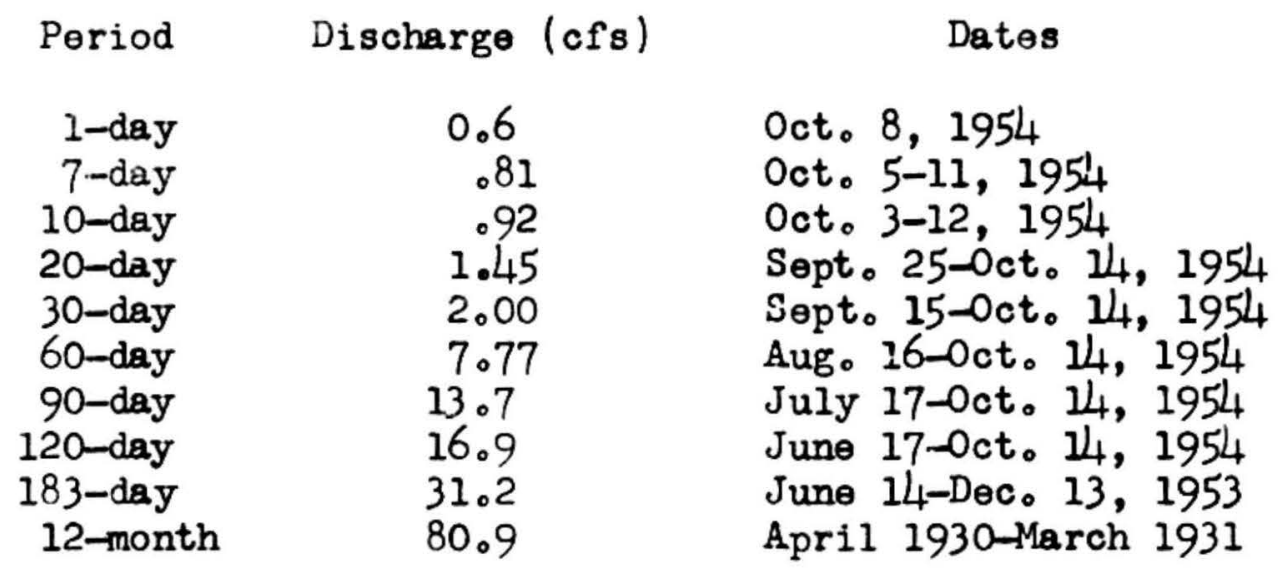

Duration of daily flow

Storage required to maintain indicated flow

Percent time indicated flow was equaled or exceeded

$\begin{array}{lc}20 & \text { mgd) } \\ 30 & 100 \\ 50 & 100 \\ 70 & 66 \\ 80 & 41 \\ 90 & 31 \\ 95 & 21 \\ 98 & 16 \\ 99 & 10 \\ 99.5 & 6.6 \\ 99.8 & 4.4 \\ 99.9 & 1.5 \\ 98 & .81\end{array}$

Regulated flow Storage required

\begin{tabular}{cc}
$(\mathrm{mgd})$ & (million gallons) \\
\hline 0.5 & 0.23 \\
.7 & 1.2
\end{tabular}

1.0

2.0

4.0

5.0

10

20

30

40

50
130

420

1,300

2,500

4,000

5,600 
Horsepen Creek at Battle Ground

Station No. 29

Location.-Lat $36^{\circ} 08^{\prime} 34^{\prime \prime}$, long. $79^{\circ} 51^{\prime} 24^{\prime \prime}$, at highway bridge half a mile downstream from U. S. Highway 220, three-quarters of a mile north of Battle Ground, Guilford County, and 2 miles upstream from mouth. Datum of gage is $737.94 \mathrm{ft}$ above mean sea level, datum of 1929, supplementary adjustment of 1936.

Drainage area.- -15.9 sq $\mathrm{mi}$.

Records available.-November 1925 to July 1931 (fragmentary prior to October 1928 ), May 1934 to September 1955.

Flow summary.-1929-30; 1934-55:

Average discharge (21 years), $14.9 \mathrm{cfs}$.

Maximum discharge, 6,400 cfs Sept. 24, 1947 (gage height, $10.36 \mathrm{ft}$ ).

Minimum discharge for indicated consecutive period:

$\begin{array}{cr}\text { Period } & \text { Discharge } \\ \text { 1-day } & 0.50 \\ 7 \text {-day } & .80 \\ 10 \text {-day } & .80 \\ 20 \text {-day } & .91 \\ 30 \text {-day } & .92 \\ 60-\text { day } & 1.97 \\ 90 \text {-day } & 2.34 \\ \text { 120-day } & 2.46 \\ \text { 183-day } & 3.71 \\ 12 \text {-month } & 7.19\end{array}$

Dates

Duration of daily flow

Storage required to maintain indicated flow

\begin{tabular}{cr}
\hline $\begin{array}{c}\text { Percent time } \\
\text { indicated flow was } \\
\text { equaled or exceeded }\end{array}$ & $\begin{array}{r}\text { Discharge } \\
\text { (mgd) }\end{array}$ \\
\hline 20 & 8.5 \\
30 & 6.2 \\
50 & 4.3 \\
70 & 2.9 \\
80 & 2.4 \\
90 & 1.8 \\
95 & 1.4 \\
98 & 1.1 \\
99 & .91 \\
99.5 & .65 \\
99.8 & .45 \\
99.9 & .41
\end{tabular}

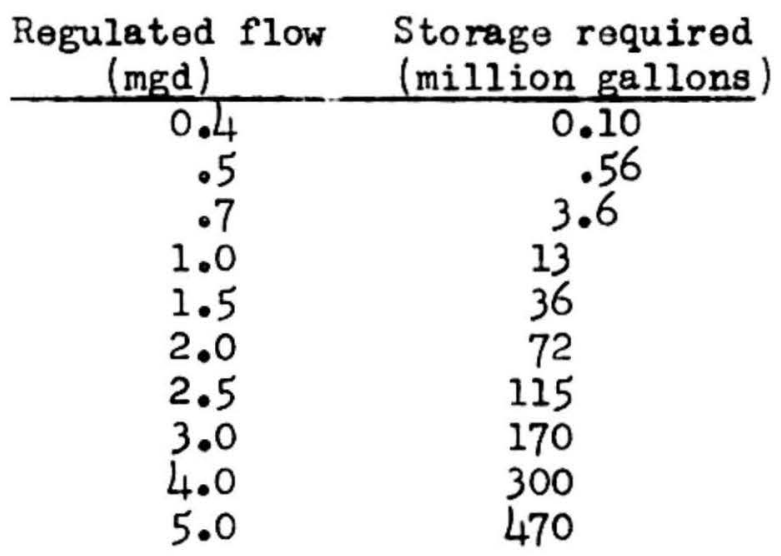

Remarks.-Total of about 9 million gallons diverted above siation by city of Greensboro during period Oct. 5, 6, 10-14, 1954 . 
Reedy Fork near Gibsonville

Station No. 30

Location.-Lat $36^{\circ} 11^{\prime}$, long. $79^{\circ} 37^{\prime}$, a cuarter of a mile downstream from Huffines Mill, 1-1/4 miles upstream from Buffalo Creek, and 6 miles Northwest of Gibsonville, Guilford County. Datum of gage is $626.88 \mathrm{ft}$ above mean sea level, datum of 1929, supplementary adjustment of 1936.

Drainage area. -133 sq $\mathrm{mi}$.

Records available. - September 1928 to September 1955.

Flow summary. - 1928-55:

Average discharge (27 years), 111 cfs.

Maximum discharge, 11,600 cfs Sept. 25, 1947 (gage height, $20.77 \mathrm{ft}$ )。

Flond of July 1916 reached a stage of 17.9 feet (discharge, 8,640 cfs).

Minimum discharge for indicated consecutive period:

\begin{tabular}{ccl} 
Period & Discharge (cfs) & \multicolumn{1}{c}{ Dates } \\
& & \multicolumn{1}{c}{} \\
1-day & 0.4 & Oct。14, 1954 \\
7-day & 1.24 & Oct。 8-14, 1954 \\
10-day & 1.42 & Oct。 5-14, 1954 \\
20-day & 1.54 & Sept。25-0ct。 14, 1954 \\
30-day & 1.90 & Sept。15-0ct。14, 1954 \\
60-day & 3.23 & Aug。12-0ct。10,1953 \\
90-day & 4.08 & Aug。5-0ct。3, 1953 \\
120-day & 5.05 & Aug。2-Nov。29, 1953 \\
183-day & 12.3 & July 4, 1953-Jan。2, 1954 \\
12-month & 53.4 & August 1953-July 1954
\end{tabular}

Duration of daily flow

Storage required to maintain indicated flow

\begin{tabular}{cc}
\hline $\begin{array}{c}\text { Percent time } \\
\text { indicated flow was } \\
\text { equaled or exceeded }\end{array}$ & $\begin{array}{c}\text { Discharge } \\
\text { (mgd) }\end{array}$ \\
\hline 20 & 100 \\
30 & 65 \\
50 & 34 \\
70 & 20 \\
80 & 15 \\
90 & 9.1 \\
95 & 5.8 \\
98 & 2.9 \\
99 & 1.5 \\
99.5 & 1.1 \\
99.8 & .76 \\
99.9 & .65
\end{tabular}

\begin{tabular}{cc}
$\begin{array}{c}\text { Regulated flow } \\
\text { (mgd) }\end{array}$ & $\begin{array}{c}\text { Storage required } \\
\text { (million gallons) }\end{array}$ \\
\hline 0.3 & 0.04 \\
.5 & .25 \\
.8 & 057 \\
1.0 & 1.4 \\
2.0 & 27 \\
4.0 & 160 \\
8.0 & 570 \\
10 & 840 \\
20 & 2,400 \\
35 & 5,400
\end{tabular}

Remarks.-Flow partly regulated since 1923 by Lake Brandt, 14 miles above station, and since 1943 by Richland Lake 12 miles above station. An average of 14.3 cfs was diverted by city of Greensboro for municipal supply, and Cone Mills diverted an average of $3.6 \mathrm{cfs}$ from Richland Lake during 1955 . 
South Buffalo Creek near Greensboro

Station No。 31

Location. -Lat $36^{\circ} 03^{\prime} 37^{\prime \prime}$, long. $79^{\circ} 43^{\prime} 33^{\prime \prime}$, at bridge on McConnell Road, 3.8 miles east of post office in Greensboro, Guilford County, and 6 miles upstream from North Buffalo Creek. Datum of gage is $696.2 \mathrm{ft}$ above mean sea level, datum of 1929, supplementary adjustment of 1936.

Drainage arez. $-33.6 \mathrm{sq} \mathrm{mi}$.

Records available. August 1928 to September 1955.

Flow summary $-1928-55$ :

Average discharge (27 years), 38.0 cfs.

Maximum discharge, 10,000 ofs July 15, 1949 (gage hoight, $11.54 \mathrm{ft}$ ).

Minimum discharge for indicated onsecutive period:

\begin{tabular}{ccl} 
Period & Discharge (cfs) & \multicolumn{1}{c}{ Dates } \\
& & \\
1-day & 0.5 & Sept. 29, 1930 \\
7-day & .80 & Sept. 28-0ct. 4, 1930 \\
10-day & .77 & Sept. 27-0ct. 6, 1930 \\
20-day & 1.08 & Sept. 25-0ct. 14, 1930 \\
30-day & 1.30 & Sept. 23-0ct. 22, 1930 \\
60-day & 1.77 & Aug.22-0ct. 2C, 1930 \\
90-day & 2.36 & Aug. 14-Nov. 11, 1930 \\
120-day & 3.65 & July 19-Nov. 15, 1930 \\
183-day & 5.06 & July 31, 1941-Jan. 29, 1942 \\
12-month & 13.8 & March 1930-February 1931
\end{tabular}

Storage required to maintain indicated flow

\begin{tabular}{cc}
$\begin{array}{c}\text { Regulated flow } \\
\text { (mgd) }\end{array}$ & $\begin{array}{c}\text { Storage required } \\
\text { (million gallons) }\end{array}$ \\
\hline 0.4 & 0.13 \\
.5 & .50 \\
.7 & 2.0 \\
1.0 & 5.5 \\
2.0 & 50 \\
3.0 & 145 \\
5.0 & 460 \\
7.0 & 880 \\
8.0 & 1,100 \\
9.0 & 1,350
\end{tabular}

Remark3.-- Low-water flow affected by sewage from Greensboro which enters above station. 
North Buffalo Creek near Greensboro

Station No. 32

Location.-Lat $36^{\circ} 07^{\prime} 13^{\prime \prime}$, long. $79^{\circ} 42^{\prime} 30^{\prime \prime}$, at highway bridge 4.2 miles uf ream from mouth, and 5.8 miles northeast of post office in Greensboro, Guilford County. Altitude of gage is $679 \mathrm{ft}$.

Drainage area. $-37.0 \mathrm{sq} \mathrm{mi}$.

Records available.-August 1928 to September 1955.

Flow summary.-1928-55:

Average discharge (27 years), 46.0 cfs.

Maximum discharge, 6,000 cfs Sept. 25, 1947 (gage height, $15.96 \mathrm{ft}$ ).

Minimum discharge for indicated consecutive period:

\begin{tabular}{rcc} 
Period & Discharge (cfs) & \multicolumn{1}{c}{ Dates } \\
1-day & 3.4 & Aug. 28, 1932 \\
7-day & 6.19 & Aug. 28-Sept. 3, 1930 \\
10-day & 6.02 & Aug. 29-Sept. 7, 1930 \\
20-day & 6.98 & Aug. 21-Sept. 9, 1930 \\
30-day & 7.70 & July 31-Aug. 29, 1932 \\
60-day & 7.64 & Aug. 29-0ct. 27, 1930 \\
90-day & 9.20 & Sept. 5-Dec. 3, 1931 \\
120-day & 11.0 & Sept. 2-Dec. 30, 1933 \\
183-day & 14.0 & June 4-Dec.3, 1933 1934 \\
12-month & 21.7 & March 1933-February 1934
\end{tabular}

Storage required to maintain indicated flow

\begin{tabular}{cc}
\hline $\begin{array}{c}\text { Regulated flow } \\
\text { (mgd) }\end{array}$ & $\begin{array}{c}\text { storage required } \\
\text { (million gallons) }\end{array}$ \\
\hline 2.5 & 0.25 \\
3.0 & 1.1 \\
4.0 & 7.0 \\
5.0 & 25 \\
6.0 & 65 \\
7.0 & 150 \\
8.0 & 270 \\
10 & 540 \\
12 & 900 \\
15 & 1,500
\end{tabular}

Remark8.-Diversion into the basin from Greensboro and Proximity Mills enters above station. 


\section{Stony Creek near Burlington}

Station No. 33

Location.-Lat $36^{\circ} 11^{\prime}$, long. $79^{\circ} 25^{\prime}$, a quarter of a mile upstream from highway bridge, half a mile upstream from Buttermilk Creek, 4-1/2 miles upstream from mouth, and 6 miles north of Burlington, Alanance County. Datum of gage is $536.3 \mathrm{ft}$ above mean sea level, datum of 1929 , supplementary adjustment of 1936.

Drainage area. - $44.2 \mathrm{sq} \mathrm{mi}$.

Records available.-July 1952 to September 1955.

Flow summary.-1952-55:

Maximum gage height, $15.26 \mathrm{ft}$ Oct. 16, 1954 (discharge not determined). Minimum discharge for indicated consecutive period:

\begin{tabular}{ccc} 
Period & Discharge (cfs) & \multicolumn{1}{c}{ Dates } \\
1-day & 0 & Sept. 12, 1954 \\
7-day & 0 & Oct. 2-8, 1954 \\
10-day & 0 & Oct. 2-11, 1954 \\
20-day & .001 & Sept. 25-0ct. 14, 1954 \\
30-day & .003 & Sept. 15-0ct. 14, 1954 \\
60-day & .010 & Oct. 6-Dec. 4, 1953 \\
90-day & .012 & Sept. 5-Dec. 3, 1953 \\
120-day & .022 & Aug. 8-Dec. 5, 1953 \\
183-day & .91 & Jul. 1-Dec. 30, 1953 \\
12-month & 19.8 & May 1953-April 1954
\end{tabular}

Storage required to maintain indicated flow

\begin{tabular}{cc}
$\begin{array}{c}\text { Regulated flow } \\
\text { (mgd) }\end{array}$ & $\begin{array}{c}\text { Storage required } \\
\text { (million gallons) }\end{array}$ \\
\hline 0.1 & 10 \\
.4 & 50 \\
.8 & 110 \\
1.2 & 180 \\
1.6 & 250 \\
2 & 330 \\
3 & 520 \\
5 & 900 \\
10 & 2,100 \\
15 & 3,600
\end{tabular}


Location.-Lat $36^{\circ} 05^{\prime}$, long. $79^{\circ} 22^{\prime}$, at town of Haw River, Alamance County, $650 \mathrm{ft}$ downstream from Southern Railway bridge, and 3 miles downstream from Stony Creek。 Datum of gage is $471.69 \mathrm{ft}$ above mean sea level, datum of 1929, supplementary adjustment of 1936.

Drainage ares. -599 sq $\mathrm{mi}$.

Records available -October 1928 to September 1955.

Flow summary. $-1928-55$ :

Average discharge (27 years) 581 cfs .

Maximum discharge, 37,000 cfs Sept, 18, 1945 (gage height, $31.10 \mathrm{ft}$ ).

Minimum discharge for indicated consecutive period:

$\begin{array}{cc}\text { Period } & \text { Discha } \\ \text { 1-day } & 5 \\ 7 \text {-day } & 164 \\ 10 \text {-day } & 18.0 \\ 20 \text {-day } & 19.5 \\ 30 \text {-day } & 23.2 \\ 60 \text {-day } & 51.7 \\ 90 \text {-day } & 57.8 \\ 120 \text {-day } & 58.4 \\ 183 \text {-day } & 97.3 \\ 12 \text {-month } & 254\end{array}$

Duration of daily flow
Dates

Sept。6, 1930

Oct. 7-13, 1954

Oct. 4-13, 1954

Sept。25-0ct。14, 1954

Sept。 15-0ct。 14, 1954

Aug。 16-0ct。14, 1954

Aug 。25-Nov。 22, 1953

July 27-Nov。 23, 1953

July 8, 1953-Jan。6, 1954

March 1933-February 1934

Storage required to maintain indicated flow

\begin{tabular}{cc}
\hline $\begin{array}{c}\text { Percent time } \\
\text { indicated flow was } \\
\text { equaled or exceeded }\end{array}$ & $\begin{array}{c}\text { Discharge } \\
\text { (mgd) }\end{array}$ \\
\hline 20 & 465 \\
30 & 320 \\
50 & 190 \\
70 & 120 \\
80 & 84 \\
90 & 52 \\
95 & 34 \\
98 & 25 \\
99 & 19 \\
99.5 & 15 \\
99.8 & 12 \\
99.9 & 9.4
\end{tabular}

Regulated flow Storage required

(mgd)

3.9

4.5

8.0

11

13

20

40

60

100

200 (million gallons)
0.6

1.3

5.0

9.4

22

160

1,100

2,700

8,000

27,000

Remarks.-Large diurnal fluctuation and some regulation at low flow caused by power plants above station. City of Burlington diverts $6.7 \mathrm{cfs}$ of which about half is returned above station as sewage. 
Haw River near Pittsboro

Station No. 35

Location.-Lat 35\%2', long. $79^{\circ} 05^{\prime}, 100 \mathrm{ft}$ upstream from Robeson Creek, 2 miles downstream from bridge on U. S. Highway 64, and 5 miles east of

Pittsboro, Chatham County. Datum of gage is $180.06 \mathrm{ft}$ above mean sea level.

Drainage area.-1,310 sq $\mathrm{mi}$, approximately.

Records available.-November 1928 to September 1955.

Flow summary.-192.8-55:

Average discharge (27 years), 1,238 cfs。

Maximum discharge 79,000 cfs Sept. 181945 (gage height, $28.58 \mathrm{ft}$ ).

Flood of August 1908 reached a stage of $32.1 \mathrm{ft}$ (di scharge, 98,000 cfs).

Minimum discharge for indicated consecutive period:

\begin{tabular}{ccc} 
Period & Discharge (cfs) & \multicolumn{1}{c}{ Dates } \\
1-day & 5.3 & Sept. 20, 1953 \\
7-day & 21.3 & Sept. 6-12, 1954 \\
10-day & 24.1 & Sept. 5-14, 1954 \\
20-day & 26.9 & Sept. 22-0ct. 11, 1954 \\
30-day & 31.9 & Sept. 5-0ct. 4, 1954 \\
60-day & 61.8 & Oct. 6-Dec. 4, 1953 \\
90-day & 74.8 & Aug. 25-Nov. 22, 1953 \\
120-day & 76.2 & July 30-Nov. 26, 1953 \\
183-day & 154 & July 10-Jan. 8, 1953 \\
12-month & 444 & Narch 1933-February 1934
\end{tabular}

Duration of daily flow

Storage required to maintain indicated flow

\section{Percent time}

indicated flow was

equaled or exceeded

$\begin{array}{ll}20 & 940 \\ 30 & 680 \\ 50 & 400 \\ 70 & 210 \\ 80 & 140 \\ 90 & 84 \\ 95 & 4.5 \\ 98 & 24 \\ 99 & 18 \\ 99.5 & 14 \\ 99.8 & 7.9 \\ 99.9 & 6.1\end{array}$

Regulated flow Storage required

$\frac{\text { (mgd) }}{3.5}$ (million gallons)

$7.6 \quad 5.2$

14

12

15

19

50

16

40

1,000

6,200

200

25,000

300

48,000

Remarks.-Considerable diurnal fluctuations and some regulation at low flow caused by power plants above station. 
New Hope River near Pittsboro

Station No. 36

Location.-Lat $35^{\circ} 44^{\prime}$, long. $79^{\circ} 02^{\prime}$, at bridge on U. S. Highway 64 , a quarter of a mile downstream from Nhiteoak Creek, and 8-3/4 miles east of Pittsboro, Chatham County. Datum of gage is $176.42 \mathrm{ft}$ above mean sea level.

Drainage area. - $285 \mathrm{sq} \mathrm{mi}$.

Records available.-January 1949 to September 1955.

Flow summary. - 1949-55:

Average discharge (6 years), $228 \mathrm{cfs}$.

Maximum discharge, 7,900 cfs Mar. 5, 1952 (gage height, $19.74 \mathrm{ft}$ ).

Flood of September 1945 . eached a stage of $27.65 \mathrm{ft}$.

Minimum discharge for indicated consecutive period:

$\begin{array}{cc}\text { Period } & \text { Discharge (cfs) } \\ & \\ \text { 1-day } & 2.2 \\ 7 \text {-day } & 2.97 \\ \text { 10-day } & 3.06 \\ \text { 20-day } & 3.67 \\ \text { 30-day } & 3.75 \\ \text { 60-day } & 6.45 \\ 90 \text {-day } & 9.69 \\ 120 \text {-day } & 11.0 \\ 183 \text {-day } & 21.5 \\ 12 \text { month } & 111\end{array}$

Dates

Sept. 4, 1953

Aug. 29-Sept. 4, 1953

Aug. 26-Sept. 4, 1953

Sept. 6-25, 1954

Sept. 3-0ct. 2, 1954

Aug. 16-0ct. 14, 1954

July 18-0ct. 15, 1954

June 18-Oct. 15, 1954

June 6-Dec. 5, 1951

December 1950-November 1951

Duration of daily flow

Storage required to maintain indicated flow

Percent time

indicated flow was

equaled or exceeded

\begin{tabular}{cc} 
d or exceeded & (mgd) \\
\hline 20 & 160 \\
30 & 90 \\
50 & 38 \\
70 & 15 \\
80 & 9.4 \\
90 & 5.2 \\
95 & 3.8 \\
98 & 2.5 \\
99 & 2.1 \\
99.5 & 1.9 \\
99.8 & 1.8 \\
99.9 & 1.6
\end{tabular}

Regulated flow Storage required

$\frac{(\mathrm{mgd})}{3.3} \quad$ (million gallons)

$4.3 \quad 80$

$5.6 \quad 150$

$7.7 \quad 300$

10

13

23

43

58

80
500

800

2,000

5,200

8,000

13,000

Remarks.-Discharge of $3.9 \mathrm{cfs}$ in 1955 diverted into basin above the station by city of Durham. 
West Fork Deep River near High Point

Station No. 37

Location.-Lat $36^{\circ} 00^{\prime} 15^{\prime \prime}$, long. $79^{\circ} 58^{\prime} 42^{\prime \prime}, 2,300$ ft upstream from highway bridge and High Point Lake, 2.3 miles west of Jamestown, and 2.5 miles northeast of High Point College, High Point, Guilford County. Altitude of gage is $758 \mathrm{ft}$.

Drainage area. - $-32.1 \mathrm{sq} \mathrm{mi}$.

Records available.-June 1923 to September 1926, July 1928 to September 1955.

Flow summary.-1923-26, 1928-55:

Average discharge ( 30 years), $31.7 \mathrm{cfs}$.

Maximum discharge 3,450 cfs Sept. 24, 1947 (gage height, $19.92 \mathrm{ft}$ ).

Minimum discharge for indicated consecutive period:

Period Discharge (cfs) Dates

$\begin{array}{cl}\text { 1-day } & 0.6 \\ \text { 7-day } & 1.07 \\ \text { 10-day } & 1.18 \\ \text { 20-day } & 1.72 \\ 30-\text { day } & 1.80 \\ 60-\text { day } & 2.96 \\ 90-\text { day } & 3.65 \\ \text { 120-day } & 4.08 \\ \text { 183-day } & 5.65 \\ 12 \text {-month } & 16.1\end{array}$

Sept. 1, 1932

Aug. 27-Sept. 2, 1932

Aug. 24-Sept. 2, 1932

Sept. 25-Oct. 14, 1954

Aug. 22-Sept. 20, 1932

Aug. 7-oct. 5, 1932

Aug. 27-Nov. 24,1941

Aug. 6-Dec. 3, 1941

Aug. 1, 19/4l-Jan. 30, $19 / 42$

February 1941-January 1942

Duration of daily flow

Storage required to maintain indicated flow

\section{Percent time}

indicated flow was

equaled or exceeded

$\begin{array}{lr}20 & 19.0 \\ 30 & 14.0 \\ 50 & 8.7 \\ 70 & 6.1 \\ 80 & 4.9 \\ 90 & 3.6 \\ 95 & 2.9 \\ 98 & 2.2 \\ 99 & 1.7 \\ 99.5 & 1.4 \\ 99.8 & .97 \\ 99.9 & .84\end{array}$

Regulated flow Storage required

$\frac{\text { (mgd) }}{0.6}$ (million gallons)

.82

1.3

1.8

3.2

4

4.9

6.3

8.5

12
1.0

6.5

20

100

190

300

500

880

1,600 
East Fork Deep River near High Point

Station No. 38

Location.-Lat $36^{\circ} 02^{\prime} 15^{\prime \prime}$, long. $79^{\circ} 56^{\prime} 46^{\prime \prime}$, at highway bridge, 3.3 miles ipstream from High Point Dam, and 5.2 miles northeast of High Point College, High Point, Guilford County. Datum of gage is $764.02 \mathrm{ft}$ above mean sea level, unadjusted.

Drainage area.-14.7 sq $\mathrm{mi}$.

Records available.-July 1928 to September 1955.

Flow summary.-1928-55:

Average discharge (27 years) $14.7 \mathrm{cfs}$.

Maximum discharge, 6,300 cfs Sept. 24, 1947 (gage height, $10.87 \mathrm{ft}$ )。

Minimum discharge for indicated consecutive period:

\begin{tabular}{ccl} 
Period & Discharge (cfs) & \multicolumn{1}{c}{ Dates } \\
1-day & 1.2 & Oct. 7, 1954 \\
7-day & 1.46 & Oct.2-8, 1954 \\
10-day & 1.48 & Sept. 28-0ct. 7, 1954 \\
20-day & 1.54 & Sept. 24-0ct. 13, 1954 \\
30-day & 1.62 & Sept.9-0ct. 8, 1954 \\
60-day & 1.97 & Sept. 7-Nov. 5, 1941 \\
90-day & 2.13 & Sept. 5-Dec. 3, 1941 \\
120-day & 2.40 & Aug. 6-Dec. 3, 1941 \\
183-day & 2.90 & Aug. 1, 1941-Jan. 30, 1942 \\
12-month & 6.70 & February 1941-January 1942
\end{tabular}

Duration of daily flow

Storage required to maintain indicated flow

\begin{tabular}{cr}
\hline $\begin{array}{c}\text { Percent time } \\
\text { indicated flow was } \\
\text { equaled or exceeded }\end{array}$ & $\begin{array}{c}\text { Discharge } \\
\text { (mgd) }\end{array}$ \\
\hline 20 & 7.6 \\
30 & 6.1 \\
50 & 4.2 \\
70 & 3.1 \\
$8 \mathrm{C}$ & 2.7 \\
90 & 2.2 \\
95 & 1.9 \\
98 & 1.6 \\
99 & 1.3 \\
99.5 & 1.1 \\
99.8 & .94
\end{tabular}

Regulated flow Storage required

$\begin{array}{cc}\text { (mgd) } & \text { (million gallons) } \\ 0.92 & 0.12 \\ 1.0 & .60\end{array}$

101

1.2

1.5

1.7

2.0

3.0

4.0

5.0

$$
2.0
$$$$
4.5
$$

20

36

67

220

400

600 


\section{Deep River near Randleman}

Station No. 39

Location.-Lat $35^{\circ} 54^{\prime} 10^{\prime \prime}$, long. $79^{\circ} 51^{\prime} 15^{\prime \prime}, 500 \mathrm{ft}$ downstream from highway bridge, a quarter of a mile downstream from Coltrane's mill, half a mile south of Guilford County line, $4-3 / 4$ miles upstream from Muddy Creek, and 7 miles north of Randleman, Randolph County. Datum of gage is $638.11 \mathrm{ft}$ above mean sea level.

Drainage area.-12/4 sq $\mathrm{mi}$.

Records available.-October 1928 to September 1955.

Flow summary.-1928-55:

Average discharge ( 27 years), 119 cfs。

Maximum discharge, 20,000 cfs Sept.25, 1947 (gage height, $32.2 \mathrm{ft}$ ).

Minimum discharge for indicated consecutive period:

Period Discharge (cfs) Dates

$\begin{array}{cc}\text { 1-day } & 1.2 \\ 7 \text {-day } & 3.94 \\ 10-\text { day } & 4.20 \\ 20 \text {-day } & 4.78 \\ 30-\text { day } & 5.08 \\ 60-\text { day } & 8.22 \\ 90-\text { day } & 9.51 \\ 120 \text {-day } & 11.44 \\ 183 \text {-day } & 13.4 \\ 12-\text { month } & 55.4\end{array}$

Nov. 12, 1933

Sept. 30-Oct. 6, 1930

Sept。30-0ct。9, 1930

Sept. 30-0ct。19, 1930

Sept.29-Oct. 28, 1930

Sept. 8-Nov. 6, 1941

Sept。5-Dec. 3, 1941

Sept. 6-Jan. 3, 1941

Aug 。 7-Feb . 5, 1941

12-month $\quad 55.4$

March 1933-February 1934

Duration of daily flow

Storage required to maintain indicated flow

Percent time

indicated flow was Discharge

equaled or exceeded (mgd)

$\begin{array}{ll}20 & 78 \\ 30 & 55 \\ 50 & 31 \\ 70 & 16 \\ 80 & 12 \\ 90 & 7.8 \\ 95 & 5.8 \\ 98 & 3.9 \\ 99 & 3.1 \\ 99.5 & 2.5 \\ 99.8 & 1.9 \\ 99.9 & 1.7\end{array}$

Regulated flow Storage required

$\frac{\text { (mgd) }}{0.9}$ (million gallons)

1.0

1.2

1.5

2.0

2.5

4.0

7.0

10

20
.27

.50

.80

1.3

2.2

21

180

490

2,0,00

Remarks. - Large diurnal fluctuation at low flow caused by Coltrane's mill. Flow slightly regulated by High Point Lake (capacity, 220,588,000 cu ft). About 3 cfs diverted by city of High Point for municipal supply. 
4

Deep River at Ramseur

Station No . 40

Location.-Lat $35^{\circ} 44^{\prime}$, long. $79^{\circ} 39,1,600 \mathrm{ft}$ downstream from railroad station at Ramseur, Randolph County and 1-1/2 miles downstream from Sandy Creek. Datum of gage is $419.50 \mathrm{ft}$ above mean sea level.

Drainage area.- $-346 \mathrm{sq} \mathrm{mi}$.

Records available. - November 1922 to September 1955.

Flow summary. -1922-55:

Average discharge ( 32 years), $343 \mathrm{cfs}$.

Maximum discharge, 43,000 cfs Sept. 18, 1945 (gage height, 34.04 ft).

Flood of August 1901 reached a stage of $28.75 \mathrm{ft}$ (discharge, $30,000 \mathrm{cfs}$ ). Minimum discharge for indicated consecutive period:

$\begin{array}{cc}\text { Period } & \text { Discharge } \\ \text { 1-day } & 0.7 \\ 7 \text {-day } & 3.57 \\ \text { 10-day } & 4.31 \\ \text { 20-day } & 608 \\ \text { 30-day } & 1.21 \\ \text { 60-day } & 9.0 \\ \text { 90-day } & 12.8 \\ \text { 120-day } & 21.4 \\ \text { 183-day } & 29.4 \\ 12-\text { month } & 130\end{array}$

Dates

Nov. 29, 1941

Oct. 19-25, 1941

Oct. 19-28, 1941

Oct. 18-Nov。 6, 1941

Oct. 8-Nov. 6, 1941

Sept. 20-Novo 18, 1941

Sept.9-Dec。7, 1941

Aug。 10-Dec。7, 1941

Aug。 5, 1941-Fob. 3, 1942

March 1933-February 1934

Duration of daily flow

Storage required to maintain indicated flow

\begin{tabular}{cc}
\hline $\begin{array}{c}\text { Porcent time } \\
\text { indicated flow was } \\
\text { equaled or exceeded }\end{array}$ & $\begin{array}{c}\text { Discharge } \\
\text { (mgd) }\end{array}$ \\
\hline 20 & 230 \\
30 & 160 \\
50 & 98 \\
70 & 52 \\
80 & 34 \\
90 & 17 \\
95 & 11 \\
98 & 6.5 \\
99 & 4.1 \\
99.5 & 2.7 \\
99.8 & 1.7 \\
39.9 & 1.3
\end{tabular}

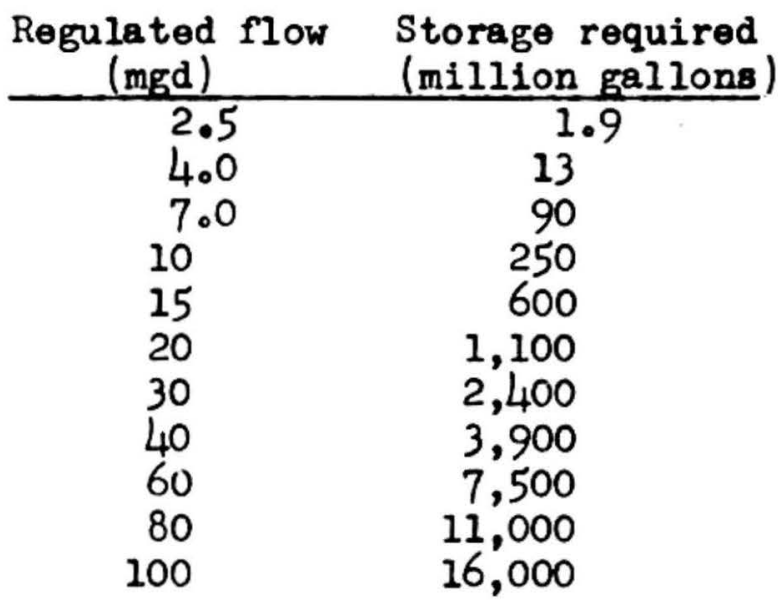

Remarks.-Large diurnal fluctuation caused by powerplants above station. Flow regulated by High Point Lake. (capacity 220,588,000 cu ft) and small powerplant reservoirs. An average of $1.0 \mathrm{cfs}$ diverted into basin above station by town of Asheboro. 


\section{Bear Creek at Robbins}

Station No. 41

Location.-Lat $35^{\circ} 26^{\prime}$, long. $79^{\circ} 36^{\prime}, 300 \mathrm{ft}$ downstream from Cabin Creek and half a mile west of Robbins, Moore County. Datum of gage is $323.23 \mathrm{ft}$ above mean sealevel, datum of 1929, supplementary adjustment of 1936.

Drainage area.-134 $8 \mathrm{qm}$.

Records available.-November 1939 to September 1955.

Flow summary.-1939-55:

Average discharge ( 16 years), 145 cfs.

Maximum discharge, 38,800 cfs Sept. 18, 1945 (gage height, $32.02 \mathrm{ft}$ ).

Minimum discharge for indicated consecutive period:

\begin{tabular}{rcl} 
Period & Discharge (cfs) & \multicolumn{1}{c}{ Dates } \\
1-day & 0 & Oct. 23, 1941 \\
7-day & .03 & Oct. 21-27, 1941 \\
10-day & .09 & Oct. 18-27, 1941 \\
20-day & .28 & Oct. 8-27, 1941 \\
30-day & .44 & Sept。 15-0ct. 14, 1954 \\
60 -day & 2.0 & Aug. 29-0ct. 27, 1941 \\
90-day & 3.0 & July 30-0ct. 27, 1941 \\
120-day & 7.2 & Aug. 7-Dec. 4, 1941 \\
183-day & 14.1 & June 5-Dec. 4, 1941 \\
12-month & 57.4 & February 1941-January 1942
\end{tabular}

Duration of daily flow

Storage required to maintain indicated flow

\begin{tabular}{cc}
\hline $\begin{array}{c}\text { Percent time } \\
\text { indicated flow was } \\
\text { equaled or exceeded }\end{array}$ & $\begin{array}{c}\text { Discharge } \\
\text { (mgd) }\end{array}$ \\
\hline 20 & 91 \\
30 & 66 \\
50 & 38 \\
70 & 19 \\
80 & 12 \\
90 & 5.5 \\
95 & 3.2 \\
98 & 1.5 \\
99 & .48 \\
99.5 & .23
\end{tabular}

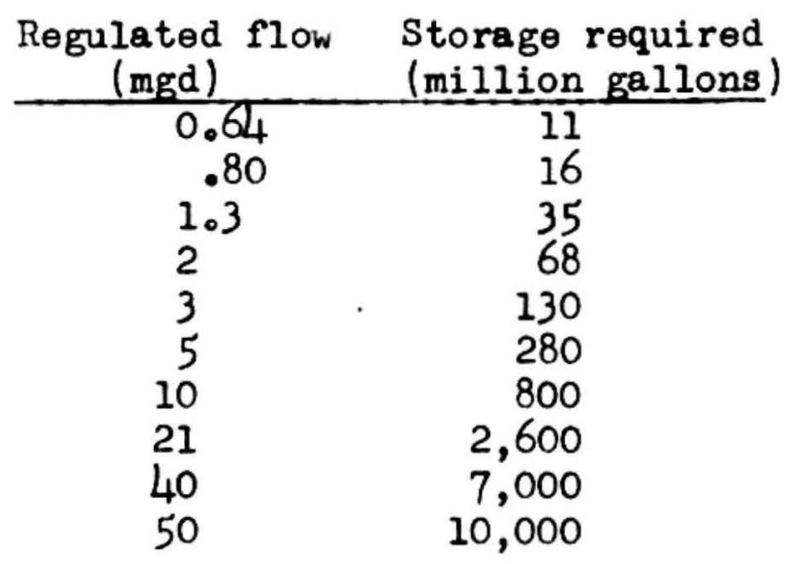

Remarks.-Town of Robbins diverts 300,000 gallons per day from gage poul below the gage. Figures herein represent flow past the gage. 
Location.-Lat $35^{\circ} 36^{\prime}$, long. $79^{\circ} 05^{\prime}, 1-1 / 2$ miles northwest of Moncure, Chatham County, 2-1/4 miles downstream from Rocky River, and 4-1/2 miles upstream from confluence with Haw River. Datum of gage is $185.88 \mathrm{ft}$ above mean sea level.

Drainage area.-1,410 sq $\mathrm{mi}$, approximately.

Records available.-July 1930 to September 1955

Flow summary.-1930-55:

Average discharge (25 years), 1,405 cfs.

Maximum discharge, 80,300 cfs Sept. 18, 1945 (gage holght, $17.20 \mathrm{ft}$ ).

Minimum discharge for indicated consecutive period:

\begin{tabular}{rcl} 
Period & Discharge (cfs) & \multicolumn{1}{c}{ Dates } \\
1-day & 6 & Oct. 9, 1954 \\
7-day & 6.6 & Oct. 8-14, 1954 \\
10-day & 7.8 & Oct. 5-14, 1954 \\
20-day & 13.0 & Nov. 3-22, 1941 \\
30-day & 13.6 & Nov. 3-Dec. 2, 1941 \\
60-day & 27.0 & Oct. 6-Dec. 4, 1941 \\
90-day & 36.1 & Sept.6-Dec. 4, 1941 \\
120-day & 57.0 & Aug. 26-Dec. 23, 1941 1912 \\
183-day & 105 & Aug. 1, 1941-Jan. 30, 1914 \\
12-month & 396 & March 1933-February 1934
\end{tabular}

Duration of daily flow

Storage required to maintain indicated flow

\section{Percent time}

indicated flow was

equaled or exceeded

\begin{tabular}{lc} 
or exceoded & $($ mgd) \\
\hline 20 & 1,000 \\
30 & 650 \\
50 & 350 \\
70 & 170 \\
80 & 110 \\
90 & 53 \\
95 & 35 \\
98 & 18 \\
99 & 14 \\
99.5 & 10 \\
99.8 & 7.4 \\
99.9 & 6.0
\end{tabular}

Regulated flow Storage required

$\frac{\text { (mgd) }}{6.5}$ (million gallons)

10

12

64

110

18

300

30

50

70

90

160

900

4,500

7,000

300
19,000

55,000

Remarks.-Diurnal fluctuation and considerable regulation at low flow caused by powerplants above station. 


\section{Cape Fear River at Lillington \\ Station No。 43}

Location.-Lat $35^{\circ} 24^{\prime}$, long. $78^{\circ} 49^{\prime}$, at bridge on U. S. Highway $15 \mathrm{~A}, 1,800 \mathrm{ft}$ downstream from Norfolk Southern Railway bridge, 0.5 mile north of Lillington, Harnett County, and 1 mile downstream from Neill Creek. Datum of gage is $105.71 \mathrm{ft}$ above mean sea level.

Drainage area. $-3,440 \mathrm{sq} \mathrm{mi}$, approximately。

Records available.-December 1923 to September 1955.

Flow summary.-1923-55:

Average discharge ( 31 years), 3,292 cfs.

Maximum discharge uncertain, occurred Sept.19, 1945 (gage height, 33.19 ft)。

Minimum discharge for indicated consecutive period:

$\begin{array}{cc}\text { Period } & \text { Discharge } \\ \text { 1-day } & 11 \\ \text { 7-day } & 28.3 \\ \text { 10-day } & 34.9 \\ \text { 20-day } & 76.5 \\ \text { 30-day } & 84.2 \\ 60 \text {-day } & 111 \\ \text { 90-day } & 154 \\ \text { 120-day } & 225 \\ \text { 183-day } & 374 \\ \text { 12-month } & 1,030\end{array}$

Dates

Duration of daily flow

Storage required to maintain indicated flow

\begin{tabular}{cc}
\hline $\begin{array}{c}\text { Percent time } \\
\text { indicated flow was } \\
\text { equaled or exceeded }\end{array}$ & $\begin{array}{c}\text { Discharge } \\
\text { (mgd) }\end{array}$ \\
\hline 20 & 2,600 \\
30 & 1,800 \\
50 & 920 \\
70 & 500 \\
80 & 320 \\
90 & 170 \\
95 & 97 \\
98 & 52 \\
99 & 45 \\
99.5 & 39 \\
99.8 & 32 \\
99.9 & 24
\end{tabular}

Regulated flow Storage required

$\frac{\text { (mgd) }}{27}$ (million gallons)

43

200

76

90

700

110

1,100

120

140

2,300

3,000

4,200

200

500

9,000

1,000
54,000

200,000

Remarks - - Large diurnal fluctuation and considerable regulation at low flow caused by powerplants above station. 


\section{Little River at Linden \\ Station No. 44}

Location.-Lat $35^{\circ} 16^{\prime}$, long. $78^{\circ} 47^{\prime}$, at bridge on U. S. Highway 15A, 1.6 miles west of Linden, Cumberland County, 2 miles upstream from Stewart Creek, and 4-1/2 miles upstream from mouth. Datum of gage is $73.10 \mathrm{ft}$ above mean sea level, datum of 1929, supplementary adjustment of 1936.

Drainage area. $-460 \mathrm{sq} \mathrm{mi}$.

Records available.-November 1928 to September 1955.

Flow summary.-1928-55:

Average discharge (26 years), 535 cfs.

Maximum discharge 13,500 cf: Sept. 18, 1945. Maximum gage hoight, 41.47

ft Sept. 19 or $20,1945$.

Flood of Sept. 21, 1928 reached a stage of $37.3 \mathrm{ft}$.

Minimum discharge for indicated consecutive period:

$\begin{array}{cc}\text { Period } & \text { Discharge } \\ \text { 1-day } & 31 \\ 7 \text {-day } & 35.4 \\ \text { 10-day } & 36.2 \\ 20 \text {-day } & 38.1 \\ 30 \text {-day } & 40.4 \\ 60 \text {-day } & 50.3 \\ 90 \text {-day } & 73.9 \\ 120 \text {-day } & 77.5 \\ 183 \text {-day } & 106 \\ 12 \text {-month } & 227\end{array}$

Duration of daily flow
Dates

Aug. 1, 1940

July 28 -Aug. 4,1940

Oct. 5-14, 1940

Sept. 28-0ct. 17, 1940

Sept. 26-Oct. 25, 1940

Sept. 1-Oct. 30, 1940

July 26-Oct. 23, 1940

July 17-Nov. 13, 1940

June 17-Dec. 16, 1940

February 1951-Jamary 1952

Storage required to maintain indicated flow

\section{Percent time}

indicated flow was Discharge

$\frac{\text { equaled or exceeded }}{20}$

30

50

70

80

90

95

98

99

99.5

99.8 (mgd)

520

390

240

130

97

65

49

36

31

28

25
Regulated flow Storage required

$\frac{(\mathrm{mgd})}{22} \quad$ (million gallons)

$26 \quad 30$

$30 \quad 110$

$35 \quad 290$

$50 \quad 1,100$

$60 \quad 1,800$

$80 \quad 4,000$

100

150

6,500

16,000 
Cape Fear River at lock 3, near Tarheel

Station No. 45

Location.-Lat $34^{\circ} 50^{\prime}$, long. $78^{\circ} 4^{\prime}$ ', $100 \mathrm{ft}$ upstroam from lock $3,7 \mathrm{miles}$ north of Tarheel, Bladen County, 9 miles upstream from Phillips Creok, and at mile 95. Datum of gage is $28.935 \mathrm{ft}$ above mean sea level, datum of 1929 , supplementary adjustment of 19.36 .

Drainage area.-4,810 sq $\mathrm{mi}$, approximately。

Kecords available.-October 1937 to September 1955.

Flow summary.--1937-55:

Average discharge ( 18 years), 4,763 cfs .

Maximum discharge not determined, occurred Sept. 22, 1945 (gage height, $43.44 \mathrm{ft}$ ).

Minimum discharge for indicated consecutive period:

$\begin{array}{rr}\text { Period } & \text { Dischar } \\ & \\ \text { 1-day } & 208 \\ \text { 7-day } & 269 \\ \text { 10-day } & 265 \\ \text { 20-day } & 305 \\ \text { 30-day } & 308 \\ \text { 60-day } & 423 \\ \text { 90-day } & 519 \\ \text { 120-day } & 656 \\ \text { 183-day } & 928 \\ \text { 12-month } & 2,360\end{array}$

Durution of daily flow
Dates

Sept. 13, 1954

Sept. 11-17, 1954

Sept,。11-20, 1954

Sept. 9-Oct. 28, 1954

Sept.9-0ct.8, 1954

Aug. 16-Oct。14, 1954

Sept. 7-Dec . 5, 1941

July 20-Nov。 16, 1953

Aug. 2-Jan 。 31, 1941

December 1950-November 1951

Storage required to maintain indicated flow

\begin{tabular}{|c|c|c|c|}
\hline $\begin{array}{l}\text { Percent time } \\
\text { indicated flow was } \\
\text { equaled or exceeded }\end{array}$ & $\begin{array}{c}\text { Discharge } \\
\text { (mgd) }\end{array}$ & $\begin{array}{c}\text { Regulated flow } \\
\text { (mgd) }\end{array}$ & $\begin{array}{l}\text { Storage required } \\
\text { (million gallons) }\end{array}$ \\
\hline 20 & 4,000 & 140 & 2.5 \\
\hline 30 & 2,800 & 150 & 8.0 \\
\hline 50 & 1,600 & 180 & 95 \\
\hline 70 & 970 & 230 & 800 \\
\hline 80 & 710 & 280 & 2,300 \\
\hline 90 & 510 & 400 & 7,400 \\
\hline 95 & 370 & 560 & 20,000 \\
\hline 98 & 270 & 700 & 35,000 \\
\hline 99 & 230 & 1,100 & 92,000 \\
\hline 99.5 & 200 & 2,000 & 330,000 \\
\hline 99.8 & 180 & & \\
\hline 99.9 & 160 & & \\
\hline
\end{tabular}

Remarks.- Slight diurnal fluctuation and some regulation at low flow caused by powerplants above station. 
Little Coharie Creek near Roseboro

Station No. 46

Location.-Lat $34^{\circ} 57^{\prime}$, long. $78^{\circ} 29^{\prime}$, at bridge on State Highway $24,1-1 / 4$ miles east of Roseboro, Sampson County, and 1-1/2 miles upstream from Bearskin Swamp. Altitude of gage is $81 \mathrm{ft}$.

Drainage area. $-96.4 \mathrm{sq} \mathrm{mi}$.

Records available.-January 1950 to September 1955.

Flow summary. - -1950-55:

Average discharge (5 years), 78.1 cfs.

Maximum discharge 1,860 cfs Sept. 6, 1955 (gage height, $9.00 \mathrm{ft}$ ).

Flood of 1924 reached a stage of $11.6 \mathrm{ft}$.

Minimum discharge for indicated consecutive period:

Period Discherge (cfs) Dates

$\begin{array}{ccl}\text { 1-day } & 0.1 & \text { Sept. 13, 1954 } \\ \text { 7-day } & .1 & \text { Oct. 1-0ct. 7, 1954 } \\ \text { 10-day } & .1 & \text { Oct. 1-10, 1954 } \\ \text { 20-day } & .13 & \text { Sept. 25-Oct. 11, 1954 } \\ \text { 30-day } & .17 & \text { Sept. 13-0ct. 12, 1954 } \\ \text { 60-day } & .88 & \text { Aug. 16-Oct. 14, 1954 } \\ \text { 90-day } & 3.8 & \text { July 28-Oct. 25, 1954 } \\ \text { 120-day } & 4.9 & \text { June 21-0ct. 18, 1954 } \\ \text { 183-day } & 11.5 & \text { May 31-Nov. 29, 1954 } \\ \text { 12-month } & 43.6 & \text { August 1950-July 1951 }\end{array}$

Duration of daily flow

Storage required to maintain indicated flow

\section{Percent time}

indicated flow was Discharge

equaled or exceeded

20
30
50
70
80
90
95
98
99

(mgd)

74

54

28

14

8.4

3.6

1.4

.25

.092
Regulated flow Storage required

\begin{tabular}{cc} 
(mgd) & (milion gallons) \\
\hline 0.6 & 15 \\
1 & 32 \\
1.5 & 57 \\
2.2 & 100 \\
3.5 & 190 \\
5 & 320 \\
7 & 520 \\
11 & 1,000 \\
20 & 2,300 \\
30 & 4,200
\end{tabular}




\section{Black River near Tomahawk}

Station No. 47

Location.-Lat $34^{\circ} 45^{\prime}$, long. $78^{\circ} 17^{\prime}$, at bridge on State Highway 411, a quarter of a mile downstream from Clear Run Swamp and 3-3/4 miles northeast of Tomahawk, Sampson County. Datum of gage is $0.39 \mathrm{ft}$ below mean sea level, detum of 1929, supplementary adjustment of 1936.

Drainage area.-680 sq mi.

Records available。-October 1951 to September 1955.

Flow summary.-1951-55:

Maximum discharge, 6,550 cfs Sept.9, 1955 (gage height, 44.16 ft).

Flood of 1928 reached a stage of $47.0 \mathrm{ft}$ (discharge, 9,000 cfs).

Minimum discharge for indicated consecutive period:

Period Discharge (cfs)

$\begin{array}{cr}\text { 1-day } & 8.9 \\ \text { 7-day } & 9.9 \\ \text { 10--day } & 10.0 \\ \text { 20-day } & 10.6 \\ \text { 30-day } & 11.9 \\ 60 \text {-day } & 13.5 \\ 90 \text {-day } & 21.6 \\ 120 \text {-day } & 32.6 \\ 183 \text {-day } & 57.3 \\ \text { 12-month } & 226\end{array}$

Duration of daily flow
Dates

Sept. 13, 1954

Oct. 9-15,1954

Oct. ' $-15,1954$

Sept. 26-0ct. 15, 1954

Sept. 16-0ct。 15, 1954

Aug. 17-0ct. 15, 1954

Aug. 7-Nov. 4, 1954

July 24-Nov。20, 1954

June 4-Dec。 3, 1954

July 1954-June 1955

Storage required to maintain indicated flow

\begin{tabular}{cc}
\hline $\begin{array}{c}\text { Percent time } \\
\text { indicated flow was } \\
\text { equaled or exceeded }\end{array}$ & $\begin{array}{c}\text { Discharge } \\
\text { (mgd) }\end{array}$ \\
\hline 20 & 460 \\
30 & 350 \\
50 & 180 \\
70 & 78 \\
80 & 46 \\
90 & 28 \\
95 & 16 \\
98 & 7.8
\end{tabular}

\begin{tabular}{cc}
$\begin{array}{c}\text { Regulated flow } \\
\text { (mgd) }\end{array}$ & $\begin{array}{c}\text { Storage required } \\
\text { (million gallons) }\end{array}$ \\
\hline $9 \cdot 4$ & 70 \\
11 & 150 \\
13 & 260 \\
15 & 370 \\
20 & 700 \\
30 & 1,500 \\
40 & 2,500 \\
60 & 5,000 \\
100 & 11,000 \\
200 & 31,000
\end{tabular}




\section{South River near Parkersburg}

Station No. 48

Location.-Lat $34^{\circ} 4^{\prime}$ ', long. $78^{\circ} 27^{\prime}$, at bridge at Bladen-Sampson County line, 1.9 miles southrest of Parkersburg, Sampson County. Datum of gage is $0.38 \mathrm{ft}$ abore moan soa lovel, datum of 1929, supplementary adjustment of 1936.

Drainege area. -382 \&q mi.

Records arailable.-October 1951 to September 1955.

Flow summary.-1951-55:

Maximum discharge, 5,000 cfs Aug. 24, 1955 (gage hoight, $64.20 \mathrm{ft}$ ). Minimum discharge for indicated consecutive period:

\begin{tabular}{ccl} 
Period & Discharge (cfs) & \multicolumn{1}{c}{ Dates } \\
1-day & 0.1 & Oct. 3, 1954 \\
7-day & 0.14 & Sept. 30-0ot. 6, 1954 \\
10-day & 0.17 & Oct. 3-12, 1954 \\
20-day & 0.22 & Sept. 25-0ot. 14, 1954 \\
30-day & 0.61 & Sept. 15-Oct. 14, 1954 \\
60-day & 1.65 & Aug. 16-0ct. 14, 1954 \\
90-day & 6.82 & Aug. 9-Nov.6, 1954 \\
120-day & 10.6 & July 24-Nov.20, 1953 \\
183-day & 26.0 & May 26-Nov. 24, 1953 \\
12-month & 157 & July 1954-June 1955
\end{tabular}

Duration of dally flow

Storage required to mintain indicated flow

\section{Percent time} indicated flow was Discharge equaled or exceeded

$\begin{array}{lc}20 & 278 \\ 30 & 207 \\ 50 & 106 \\ 70 & 28 \\ 80 & 14 \\ 90 & 6.1 \\ 95 & 2.6 \\ 98 & .43 \\ 99 & .17\end{array}$

Regulated flow Storage required

\begin{tabular}{cc} 
(mgd) & (million gallons) \\
\hline 1.0 & 20 \\
2.0 & 64 \\
4.0 & 170 \\
6.0 & 310 \\
10 & 6,0 \\
15 & 1,100 \\
20 & 1,700 \\
40 & 4,400 \\
80 & 11,000 \\
120 & 20,000
\end{tabular}




\section{Colly Creek near Kelly}

Station No. 49

Location. -Lat $34^{\circ} 28^{\prime}$, long. $78^{\circ} 15^{\prime}$, at bridge on State Highway 53,4 miles east of Kelly, Bladen County, and 6-3/4 miles upstream from mouth. Datum of gage is $15.4 \mathrm{ft}$ above mean sea level, unadjusted.

Drainage area. $-85.2 \mathrm{sq} \mathrm{mi}$.

Records available.-January 1950 to September 1955.

Flow summary.-1950-55:

Average discharge ( 5 years), $69.1 \mathrm{cfs}$.

Maximum discharge, 910 cfs Sept. 22, 1955 (gage height, $7.20 \mathrm{ft}$ ).

Floods of 1908,1928 , and September 1945 reached stages of $11.1,7.7$ and $10.2 \mathrm{ft}$ respectively.

Minimum discharge for indicated consecutive period:

Period Discharge (cfs) Dates

$\begin{array}{ccl}\text { 1-day } & 0 & \text { Oct. 27, 1951 } \\ \text { 7-day } & 0 & \text { Oct. 27-Nov. 2, 1951 } \\ \text { 10-day } & 0 & \text { July 23-Aug. 1, 1953 } \\ \text { 20-day } & 0 & \text { Oct. 12-31, 1953 } \\ \text { 30-day } & 0 & \text { Oct. 12-Nov. 10, 1953 } \\ \text { 60-day } & 0 & \text { Aug. 9-Oct. 6, 1954 } \\ 90-\text { day } & .03 & \text { Aug. 2-Oct. 30, 1954 } \\ \text { 120-day } & .23 & \text { July 31-Nov. 27, 1954 } \\ \text { 183-day } & .99 & \text { June 6-Dec. 5, 1954 } \\ \text { 12-month } & 20.8 & \text { June 1954-May 1955 }\end{array}$

Duration of daily flow

Storage required to maintain indicated flow

\begin{tabular}{cc}
\hline $\begin{array}{c}\text { Percent time } \\
\text { indicated flow was } \\
\text { equaled or exceeded }\end{array}$ & $\begin{array}{c}\text { Discharge } \\
\text { (mgd) }\end{array}$ \\
\hline 20 & 71 \\
30 & 52 \\
50 & 21 \\
70 & 3.2 \\
80 & .91 \\
90 & .04
\end{tabular}

\begin{tabular}{cc}
$\begin{array}{c}\text { Regulated flow } \\
\text { (mgd) }\end{array}$ & $\begin{array}{c}\text { Storage required } \\
\text { (million gallons) }\end{array}$ \\
\hline 0.5 & 43 \\
.8 & 78 \\
1.2 & 130 \\
2 & 250 \\
4 & 600 \\
6 & 1,000 \\
8 & 1,500 \\
10 & 2,000 \\
$\frac{14}{20}$ & 3,000 \\
& 4,800
\end{tabular}


Northeast Cape Fear River near Chinquapin

Station No. 50

Location.-Lat $34^{\circ} 49^{\prime}$, long. $77^{\circ} 50^{\prime}$, $540 \mathrm{ft}$ downstream from bridge on State Highway 4l, half a mile downstream from Muddy Creok, and 1-1/4 miles west of Chinquapin, Duplin County. Datum of gage is $17.28 \mathrm{ft}$ above mean sea level.

Drainage area. -600 sq $\mathrm{mi}$.

Records available.-July 1940 to September 1955.

Flow summary.-1940-55:

Average discharge ( 15 years), $64_{4}$ cfs.

Maximum discharge, 15,200 cfs Sept. 22, 1955 (gage height, 17.97 ft).

Flood of 1908 reached a stage of $22.6 \mathrm{ft}$.

Minimum discharge for indicated consecutive period:

\begin{tabular}{ccl} 
Period & Discharge (cfs) & \multicolumn{1}{c}{ Dates } \\
1-day & 5.3 & Oct. 10, 1954 \\
7-day & 5.47 & Oct. 8-14, 1954 \\
10-day & 5.81 & Oct. 5-14, 1954 \\
20-day & 6.88 & Oct. 3-22, 1954 \\
30-day & 7.44 & Sept. 29-0ct. 28, 1954 \\
60-day & 8.114 & Sept.9-Nov.7, 1954 \\
90-day & 9.89 & Aug. 11-Nov. 8, 1954 \\
120-day & 11.6 & July 29-Nov. 25, 1954 \\
183-day & 18.6 & June 18-Dec. 17, 1954 \\
12-month & 97.0 & June 1954-May 1955
\end{tabular}

Duration of daily flow

Storage required to mintain indicated flow

\begin{tabular}{cc}
\hline $\begin{array}{c}\text { Percent time } \\
\text { indicated fl.ow was } \\
\text { equaled or exceeded }\end{array}$ & $\begin{array}{c}\text { Discharge } \\
\text { (mgd) }\end{array}$ \\
\hline 20 & 610 \\
30 & 400 \\
50 & 220 \\
70 & 84 \\
80 & 47 \\
90 & 21 \\
95 & 13 \\
98 & 8.4 \\
99 & 6.8 \\
99.5 & 5.5 \\
99.8 & 4.2
\end{tabular}

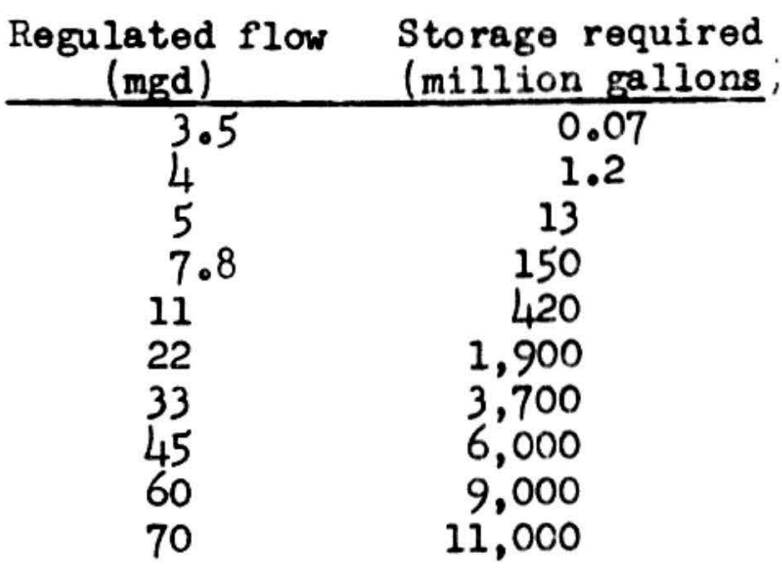




\section{Waccamaw River at Freeland}

Station No. 51

Location.-Lat $34^{\circ} 05^{\prime} 4^{\prime \prime}$, long. $78^{\circ} 32^{\prime} 56^{\prime \prime}$, $150 \mathrm{ft}$ downstream from New Britton Bridge on State Highway 130, 1 mile southwest of Freeland, Brunswick

County, and 7 miles downstream from Juniper Creek. Datum of gage is 15.52 ft above mean sea level, datum of 1929, supplementary adjustment of 1936.

Drainage area.-626 sq mi.

Records available.-July 1939 to September 1955.

Flow summary.-1939-55:

Average discharge ( 16 years), 611 cfs。

Maximum discharge, 10,200 cfs Sept. 25, 1955. Maximum gage height, 16.63 ft Sept. 26, 1955.

Minimum discharge for indicated consecutive period:

$\begin{array}{cc}\text { Period } & \text { Di scharge } \\ \text { 1-day } & 0.1 \\ 7 \text {-day } & .10 \\ 10 \text {-day } & .10 \\ 20 \text {-day } & .16 \\ 30 \text {-day } & .28 \\ 60 \text {-day } & .37 \\ 90 \text {-day } & .82 \\ 120 \text {-day } & .90 \\ 183 \text {-day } & 4.26 \\ 12-\text { month } & 145\end{array}$

Duration of daily flow
Storage required to maintain indicated flow

Aug. 30, 1954

Oct. 4-10, 1954

Oct. 4-13, 1954

Sept。25-0ct。 14, 1954

Sept。15-0ct。14, 1954

Aug. 16-0ct。 14, 1954

Aug. 22-Nov。 19, 1954

Aug。11-Dec。11, 1954

June 29-Dec。28, 1954

March 1940-February 1941

Percent time

indicated flow was Discharge

equaled or exceeded

20

30

50

70

80

90

95

98

99

99.5

99.8 (mgd)

620

400

180

45

21

5.0

.91

.63

.54

.47

.32
Regulated flow Storage required

\begin{tabular}{cc} 
(mgd) & (million gallons) \\
\hline 0.13 & 0.7 \\
.20 & 3.5 \\
.30 & 9 \\
.60 & 30 \\
1.0 & 62 \\
3.0 & 300 \\
5.0 & 550 \\
40 & 7,500 \\
70 & 15,000 \\
100 & 25,000
\end{tabular}




\section{Yadkin River at Patterson}

Station No. 52

Location.-Lat $35^{\circ} 59^{\prime} 30^{n}$, long $81^{\circ} 33^{\prime} 30^{\prime \prime}, 200 \mathrm{ft}$ upstream from bridge on State Highway 268, half a mile south of Patterson, Caldwell County, three quarters of a mile upstream from Warrior Creek, and 2 miles downstream from Walnut Branch. Datum of gage is 1,212.47 ft above mea sea level, unadjusted.

Drainage area. $-28.8 \mathrm{sq} \mathrm{mi}$.

Records available.-November 1939 to September 1955.

Flow summary -1939-55:

Average discharge ( 16 years), 44.4 cfs

Maximum discharge, 16,200 cfs Aug. 13, 1940 (gage height, $12.70 \mathrm{ft}$ ).

Minimum discharge for indicated consecutive period:

\begin{tabular}{ccc} 
Period & Discharge (cfs) & \multicolumn{1}{c}{ Dates } \\
1-day & 5.3 & Sept. 30, 1754 \\
7-day & 5.73 & Sept. 25-Oct. 1, 1954 \\
10-day & 6.04 & Sept. 22-Oct. 1, 1954 \\
20-day & 6.48 & Sept. 12-0ct. 1, 1954 \\
30-day & 6.84 & Sept. 2-0ct. 1, 1954 \\
60-day & 7.68 & Sept. 3-Nov. 1, 1954 \\
90-day & 8.93 & Aug. 5-Nov. 2, 1954 \\
120-day & 9.22 & July 20-Nov. 16, 1954 \\
183-day & 11.1 & June 29-Dec. 28, 1954 \\
12-month & 23.4 & July 1954-June 1955
\end{tabular}

Duration of daily Now

Storage required to maintain indicated flow

\begin{tabular}{cc}
\hline $\begin{array}{c}\text { Percent time } \\
\text { indicated flow was } \\
\text { equaled or exceeded }\end{array}$ & $\begin{array}{c}\text { Discharge } \\
\text { (mgd) }\end{array}$ \\
\hline 20 & 36 \\
30 & 30 \\
50 & 21 \\
70 & 15 \\
80 & 12 \\
90 & 9.4 \\
95 & 7.8 \\
98 & 6.2 \\
99 & 5.0 \\
99.5 & 4.5 \\
99.8 & 4.1 \\
99.9 & 3.9
\end{tabular}

Regulated flow Storage required

$\frac{\text { (mgd) } \quad \text { (million gallons) }}{4.0}$

$5.0 \quad 18$

$6.0 \quad 60$

$7.0 \quad 140$

$8.0 \quad 250$

$9.0 \quad 390$

$10 \quad 550$

12 . 910

131,100

$15 \quad 1,500$ 
Reddies River at North Wilkesboro

Station No。 53

Location - Lat $36^{\circ} 10^{\prime}$, long. $81^{\circ} 10^{\prime}, 400$ ft upstream from highray bridge, 1-1/4 miles northwest of North Wilkesboro, Wilkes County, 1-1/4 miles upstream from North Nilkesboro municipal dam, and 2 miles upstream from mouth. Datum of gage is $978.62 \mathrm{ft}$ above mean sea level, datum of 1929, supplementary adjustment of 1936.

Drainage area. - 93.9 sq mi.

Records available.-December 1939 to September 1955.

Flow summary.-1939-55:

Average discharge ( 16 years), 135 cfs.

Maximum discharge, 27,000 cfs Aug。14, 1940 (gage height, $22.02 \mathrm{ft}$ )。

Minimum discharge for indicated consecutive period:

\begin{tabular}{|c|c|c|}
\hline Period & Discharge (cfs) & Dates \\
\hline $\begin{array}{l}\text { 1-day } \\
\text { 7-day } \\
\text { 10-day } \\
\text { 20-day } \\
\text { 30-day } \\
60-\text { day } \\
\text { 90-day } \\
120-\text { day } \\
183 \text {-day } \\
\text { 12-month }\end{array}$ & $\begin{array}{l}23 \\
25.0 \\
25.8 \\
29.8 \\
29.1 \\
32.6 \\
40.2 \\
41.1 \\
46.6 \\
74.3\end{array}$ & $\begin{array}{l}\text { Aug. 17, 1954 } \\
\text { Aug. 11-17, 1954 } \\
\text { Aug。 9-18, 1954 } \\
\text { Sept。 12-Oct。 1, } 1954 \\
\text { Sept. 15-Oct。 14, } 1954 \\
\text { Aug. 31-Oct. 29, 1954 } \\
\text { Aug. 29-Nov. 27, 1954 } \\
\text { July 15-Nov. 11, 1954 } \\
\text { June 6-Dec。 5, 1954 } \\
\text { April 1954-March } 1955\end{array}$ \\
\hline
\end{tabular}

Duration of daily flow

Storage required to maintain indicated flow

\begin{tabular}{cc}
\hline $\begin{array}{c}\text { Percent time } \\
\text { indicated flow was } \\
\text { equaled or exceeded }\end{array}$ & $\begin{array}{c}\text { Discharge } \\
\text { (mgd) }\end{array}$ \\
\hline 20 & 100 \\
30 & 88 \\
50 & 71 \\
70 & 54 \\
80 & 47 \\
90 & 39 \\
95 & 34 \\
98 & 26 \\
99 & 22 \\
99.5 & 19 \\
99.8 & 18 \\
99.9 & 17
\end{tabular}

Regulated flow Storage required (mgd)

18

20

25

30

35

40

43

45

47

50 (milition gallons)

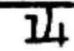

50

250

600

1,100

1,800

2,400

2,800

3,200

4,000 
Yadkin River at Wilkesboro

Station No. 54

Location.-Lat $36^{\circ} 09^{\prime}$, long. $81^{\circ} 09^{\prime}, 150 \mathrm{ft}$ upstream from bridge on U.S. Highway 421 between North Wilkesboro and Wilkesboro, $150 \mathrm{ft}$ downstreem from Reddies River, and half a mile northeast of Wilkesboro, Wilkes County. Datum of gage is $942.35 \mathrm{ft}$ above mean sea level, datum of 1929, supplementary adjustment of 1936.

Drainage area. -493 sq $\mathrm{mi}$.

Records available. April 1903 to June 1909, October 1920 to September 1955.

Flow summary. $-1903-9,1920-55$ :

Average discharge 1903-6, 1920-55 (38 years), 771 cfs.

Maximum discharge, 160,000 cfs Aug. 14,1940 (gage height, $37.6 \mathrm{ft}$ ).

Minimum discharge for indicated consecutive period:

\begin{tabular}{|c|c|c|}
\hline Period & Discharge (cfs) & Dates \\
\hline 1-day & 113 & Sept. 30, 1954 \\
\hline $\begin{array}{r}7 \text {-day } \\
10 \text {-day }\end{array}$ & $\begin{array}{l}120 \\
123\end{array}$ & $\begin{array}{l}\text { Sept. } 24,-30,1954 \\
\text { Oct. } 5-14.1954\end{array}$ \\
\hline $\begin{array}{l}10 \text {-day } \\
20-d a y\end{array}$ & 128 & Sept。25-0ct。 14, 1954 \\
\hline 30-day & 130 & 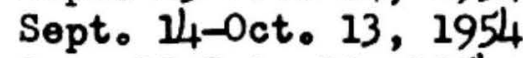 \\
\hline 60-day & 150 & Aug. 31-0ct. 29, 1954 \\
\hline 90-day & 183 & Aug. 4-Nov. 1, 1954 \\
\hline 20 -day & 194 & July 19-Nov。 15,1954 \\
\hline 83-day & 240 & June 6-Dec. 5, 1954 \\
\hline 12-month & 417 & July 1954-June 1955 \\
\hline
\end{tabular}

Duration of daily flow

Storage required to maintain indicated flow

\begin{tabular}{cc}
\hline $\begin{array}{c}\text { Percent time } \\
\text { indicated flow was } \\
\text { equaled or exceeded }\end{array}$ & $\begin{array}{c}\text { Discharge } \\
\text { (mgd) }\end{array}$ \\
\hline 20 & 600 \\
30 & 500 \\
50 & 390 \\
70 & 290 \\
80 & 250 \\
90 & 200 \\
95 & 170 \\
98 & 140 \\
99 & 130 \\
99.5 & 110 \\
99.8 & 91 \\
99.9 & 81
\end{tabular}

\begin{tabular}{cc}
$\begin{array}{c}\text { Regulated flow } \\
\text { (mgd) }\end{array}$ & $\begin{array}{c}\text { Storage required } \\
\text { (million gallons) }\end{array}$ \\
\hline 80 & 15 \\
90 & 160 \\
100 & 540 \\
120 & 1,500 \\
140 & 2,900 \\
170 & 5,600 \\
200 & 9,800 \\
230 & 15,000 \\
250 & 19,500 \\
270 & 25,000
\end{tabular}

Remarks.-Slight diurnal fluctuation at low flow caused by powerplant on Reddies River 1 mile above station. 


\section{Fisher River near Copeland}

Station No. 55

Location.-Lat $36^{\circ} 20^{\prime}$, long. $80^{\circ} 40^{\prime}, 500$ ft upstream from bridge on State Highway 268, 1 mile upstream from Cody Creek, and 2 miles northwest of Copeland, Surry County. Altitude of gage is $913 \mathrm{ft}$ ( $\mathrm{b}_{\mathrm{y}}$ barometer).

Drainage area. - 121 sq $\mathrm{mi}$

Records available.-October 1931 to September 1955.

Flow summary.-1931-55:

Average discharge (2/4 years), $178 \mathrm{cfs}$.

Maximum discharge, 27,300 cfs Aug. 14,1940 (gage height, 18.4 feet).

Minimum discharge for indicated consecutive period:

\begin{tabular}{|c|c|c|}
\hline Period & Discharge (cfs) & Dates \\
\hline $\begin{array}{l}\text { 1-day } \\
7 \text {-day } \\
\text { 10-day } \\
\text { 20-day } \\
\text { 30-day } \\
60-\text { day } \\
\text { 90-day } \\
120-\text { day } \\
183 \text {-day } \\
\text { 12-month }\end{array}$ & $\begin{array}{l}17 \\
20.6 \\
21.4 \\
25.3 \\
27.9 \\
38.6 \\
44.9 \\
52.2 \\
65.6 \\
98.9\end{array}$ & $\begin{array}{l}\text { Sept. 18, 1954 } \\
\text { Sept. 13-19, 1954 } \\
\text { Sept.11-20, 1954 } \\
\text { Sept. 11-30, 1954 } \\
\text { Sept. 1-30, 1954 } \\
\text { Aug. 11-0ct. 9, 1954 } \\
\text { July 17-0ct. 11, 195/4 } \\
\text { July 12-Nov。 8, 1954 } \\
\text { Aug. 25, 1933-Feb. 23, } 1934 \\
\text { February 1941-January } 1942\end{array}$ \\
\hline
\end{tabular}

Duration of daily flow

\begin{tabular}{cc}
\hline $\begin{array}{c}\text { Percent time } \\
\text { indicated flow was } \\
\text { equaled or exceeded }\end{array}$ & $\begin{array}{c}\text { Discharge } \\
\text { (mgd) }\end{array}$ \\
\hline 20 & 110 \\
30 & 110 \\
50 & 87 \\
70 & 64 \\
80 & 55 \\
90 & 42 \\
95 & 36 \\
98 & 27 \\
99 & 23 \\
99.5 & 20 \\
99.8 & 17 \\
99.9 & 15
\end{tabular}

Storage required to maintain indicated flow

Remarks.-Records collected at station near Dobson (drainage area, 109 sq mi) $1920-32$.

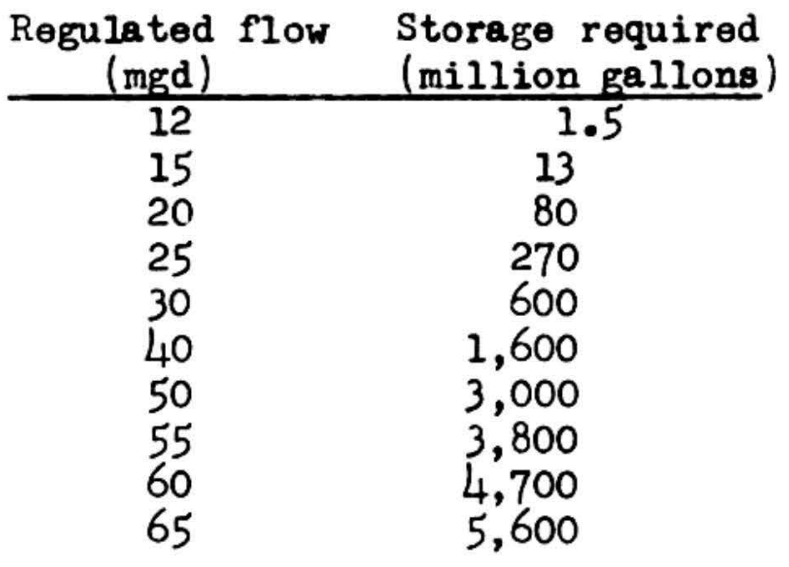




\section{Forbush Creek near Yadkinville}

Station No. 56

Location.-Lat $36^{\circ} 08^{\prime}$, long. $80^{\circ} 33^{\prime}$, $900 \mathrm{ft}$ upstream from highway bridge, three-quarters of a mile north of Forbush Church, 2-3/4 miles upstream from Logan Creek, 3-1/2 miles upstream from mouth, and 6 miles east of Yadkinville, Yadkin County。 Altitude of gage is $728 \mathrm{ft}$ (by barometer).

Drainage area. $-21.7 \mathrm{sq} \mathrm{mi}$.

Records available.-April 1940 to September 1955.

Flow summary.-1940-55:

Average discharge ( 15 years), 21.2 cfs.

Maximum di scharge, 2,450 cfs Sept。30, 1944 (gage height, $11.02 \mathrm{ft}$ )。

Minimum discharge for indicated consecutive period:

\section{Period Discharge (cfs) Dates}

$\begin{array}{cc}\text { 1-day } & 1.3 \\ 7-\text { day } & 1.50 \\ \text { 10-day } & 1.66 \\ \text { 20-day } & 1.82 \\ \text { 30-day } & 1.79 \\ 60-\text { day } & 3.41 \\ \text { 90-day } & 4.54 \\ 120 \text {-day } & 5.38 \\ \text { 183-day } & 7.62 \\ 12 \text {-month } & 12.2\end{array}$

Sept。21, 1955

Sept. 17-23, 1955

Sept.10-19, 1954

Sept。 18-oct。7, 1954

Sept。9-0ct。8, 1954

Aug. 16-0ct。14, 1954

July $17-0 c t$. 14, 1954

Juno 17-Oct。14, 1954

June 20-Dec。19, 1951

February 19/4l-January 1942

Duration of daily flow

Storage required to maintain indicated flow

\begin{tabular}{cc}
\hline $\begin{array}{c}\text { Percent time } \\
\text { indicated flow was } \\
\text { equaled or exceeded }\end{array}$ & $\begin{array}{c}\text { Discharge } \\
\text { (mgd) }\end{array}$ \\
\hline 20 & 15 \\
30 & 12 \\
50 & 9.0 \\
70 & 6.7 \\
80 & 5.5 \\
90 & 4.2 \\
95 & 3.3 \\
98 & 2.5 \\
99 & 2.0 \\
99.5 & 1.6
\end{tabular}

\begin{tabular}{cc}
$\begin{array}{c}\text { Regulated flow } \\
\text { (mgd) }\end{array}$ & $\begin{array}{c}\text { Storage required } \\
\text { (million gallons) }\end{array}$ \\
\hline 1.0 & 0.3 \\
1.3 & 5.0 \\
1.5 & 11 \\
2.0 & 32 \\
3.0 & 93 \\
4.0 & 170 \\
5.0 & 260 \\
6.0 & 360 \\
7.0 & 470 \\
8.0 & 600
\end{tabular}

Remarks.-Diurnal fluctuation and slight regulation for short periods during low flow caused by mills above station. 


\section{Yadkin River at Yadkin College}

Station No. 57

Location.-Lat $35^{\circ} 51^{\prime} 24^{\prime \prime}$, long. $80^{\circ} 23^{\prime} 09^{\prime \prime}$, $80 \mathrm{ft}$ downstream from bridge on U. S. Highway $64,1-1 / 2$ miles south of Yadkin College, Davidson County, and 6-1/4 miles downstream from Reedy Creek. Datum of gage is $638.65 \mathrm{ft}$ above mean sea level.

Drainage area. $-2,280 \mathrm{sq} \mathrm{mi}$, approximately.

Records available.-July 1928 to September 1955.

Flow summary.-1928-55:

Average discharge (27 years), 2,849 cfs.

Maximum discharge, 80,200 cfs Aug 15, 1940 (gage height, $33.75 \mathrm{ft}$ ); maximum stage known, $36.3 \mathrm{ft}$ during July 1916 (discharge 94,300 cfs).

Minimum discharge for indicated consecutive period:

\begin{tabular}{rcl} 
Period & Discharge (cfs) & \multicolumn{1}{c}{ Dates } \\
1-day & 330 & Oct. 9, 1954 \\
7-day & 385 & Oct. 8-14, 1954 \\
10-day & 397 & Oct. 5-14, 1954 \\
20-day & 422 & Sept.25-0ct. 14, 1954 \\
30-day & 431 & Sept. 15-0ct. 14, 1954 \\
60-day & 637 & Aug. 16-Oct. 14, 1954 \\
90-day & 720 & July 17-0ct. 14, 1954 \\
120-day & 845 & June 17-0ct. 14, 1954 \\
183-day & 1,106 & May 30-Nov。 28, 1954 \\
12-month & 1,591 & Apri1 1930-May 1931
\end{tabular}

Duration of daily flow

Storage required to maintain indicated flow

Percent time

indicated flow was Discharge

equaled or exceeded (mgd)

$\begin{array}{lr}20 & 2,200 \\ 30 & 1,900 \\ 50 & 1,400 \\ 70 & 1,100 \\ 80 & 910 \\ 90 & 720 \\ 95 & 610 \\ 98 & 480 \\ 99 & 410 \\ 99.5 & 360 \\ 99.8 & 300 \\ 99.9 & 270\end{array}$

Regulated flow Storage required

$\frac{\text { (mgd) (million gallons) }}{20}$

250

80

300

400

650

500

600

700

800

1,000
4,000

8,700

15,000

23,000

32,000

57,000

Remarks.-Diurnal fluctuation during low flow caused by small powerplant with

little storage capacity 10 miles upstream from station. 
Location.-Lat $35^{\circ} 54^{\prime}$, long. $80^{\circ} 48^{\prime}, 1,000 \mathrm{ft}$ downstream from bridge on U. S. Highway 21 at Turnersburg, Iredell County, I mile downstream from Mud Creek, and 1-1/2 miles upstream from mouth. Datum of gage is $724.10 \mathrm{ft}$ above mean sea level, datum of 1929, supplementary adjustment of 1936.

Drainage area. $-85.5 \mathrm{sq} \mathrm{mi}$.

Records available.-April 1940 to September 1955.

Flow summary. - $1940-55$ :

Average discharge ( 15 years), 107 cfs.

Maximum discharge, 6,080 cfs Jan. 22, 1954 (gage height, $13.70 \mathrm{ft}$ ).

A stage of about $18 \mathrm{ft}$ was reached by flood sometime during the years 1936 to 1938 .

Minimum discharge for indicated consecutive period:

$\begin{array}{cc}\text { Period } & \text { Discharge } \\ \text { 1-day } & 13 \\ 7 \text {-day } & 14.7 \\ 10 \text {-day } & 14.8 \\ 20 \text {-day } & 16.1 \\ 30 \text {-day } & 16.1 \\ 60 \text {-day } & 20.3 \\ 90 \text {-day } & 24.6 \\ \text { 120-day } & 29.2 \\ 183 \text {-day } & 37.2 \\ 12 \text {-month } & 63.0\end{array}$

Duration of daily flow
Storage required to maintain indicated flow

\begin{tabular}{cc}
\hline $\begin{array}{c}\text { Percent time } \\
\text { indicated flow was } \\
\text { equaled or exceeded }\end{array}$ & $\begin{array}{c}\text { Discharge } \\
\text { (mgd) }\end{array}$ \\
\hline 20 & 79 \\
30 & 66 \\
50 & 50 \\
70 & 37 \\
80 & 32 \\
90 & 26 \\
95 & 22 \\
98 & 17 \\
99 & 14 \\
99.5 & 12
\end{tabular}

Sept. 19, 1954

Sept. 13-19, 1954

Sept. 10-19, 1954

Sept.1-20, 1954

Sept.8-0ct. 7, 1954

Aug. 30-0ct。28, 1954

Aug. 11-Nov。 8, 1954

July 6-Nov. 2, 1954

June 6-Dec. 5, 1954

Juiy 1954-June 1955

Remarks.-Considerable diurnal fluctuation at low flow caused by mills above station affected minimum of record. Slight fluctuation in recent years. 


\section{Station No。 59}

Location.-Lat $35^{\circ} 51^{\prime}$, long. $80^{\circ} 40^{\prime}$, at downstream side of highway bridge, 1 mile upstream from Little Creek, 4 miles downstream from Fifth Creek, 4-1/2 miles upstream from Hunting Creek, and 6-1/2 miles southwest of Mocksville, Davie County。 Altitude of gage is $660 \mathrm{ft}$ (by barometer).

Drainage area.-313 sq $\mathrm{mi}$.

Records available -October 1938 to September 1955.

Flow summary.-1938-55:

Average discharge ( 17 yoars), 307 cfs.

Maximum discharge, 9,240 cfs Jan. 23, 1954 (gage hoight, $16.73 \mathrm{ft}$ ); maximum stage known, 22.6 ft Oct。 $3,1929$.

Minimum discharge for indicated consecutive period:

\begin{tabular}{|c|c|c|}
\hline Period & Discharge (cfs) & Dates \\
\hline $\begin{array}{l}\text { 1-day } \\
\text { 7-day } \\
\text { 10-day } \\
\text { 20-day } \\
\text { 30-day } \\
60-\text { day } \\
\text { 90-day } \\
120 \text {-day } \\
183 \text {-day } \\
\text { 12-month }\end{array}$ & $\begin{array}{l}35 \\
39.3 \\
40.3 \\
41.8 \\
43.0 \\
56.7 \\
67.5 \\
78.6 \\
99.5 \\
177\end{array}$ & 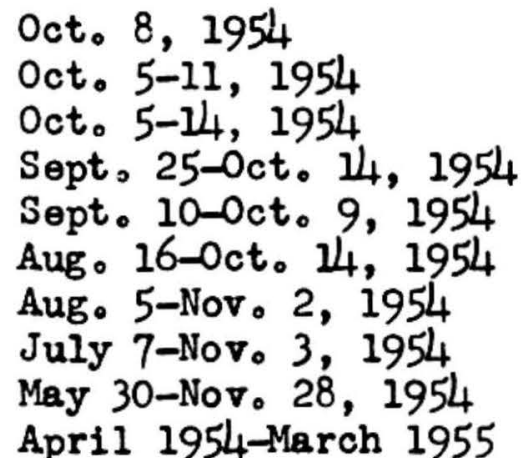 \\
\hline
\end{tabular}

Duration of daily flow

Storage required to maintain indicated flow

\begin{tabular}{cc}
\hline $\begin{array}{c}\text { Percent time } \\
\text { indicated flow was } \\
\text { equaled or exceeded }\end{array}$ & $\begin{array}{c}\text { Discharge } \\
\text { (mgd) }\end{array}$ \\
\hline 20 & 230 \\
30 & 190 \\
50 & 140 \\
70 & 110 \\
80 & 90 \\
90 & 72 \\
95 & 61 \\
98 & 49 \\
99 & 40 \\
99.5 & 30 \\
99.8 & 27 \\
99.9 & 26
\end{tabular}

Regulated flow Storage required

$\frac{\text { (mgd) }}{25}$ (million gallons)

30

40

58

50

60

70

80

90

100

115
550

1,200

2,100

3,100

4,200

5,600

7,000

9,600 
Location.-Lat $36^{\circ} 00^{\prime}$, long. $80^{\circ} 44^{\prime}$, at downstream side of highway bridge, three-quarters of a mile downstream from Kennedy Creek, 1 mile east of Houstonville, Iredell County, 2 miles downstream from U. S. Highway 21 , and 3-1/2 miles northeast of Harmony. Altitude of gage is $731 \mathrm{ft}$ (by barometer).

Drainage area. $-153 \mathrm{sq} \mathrm{mi}$.

Records available.-January 1951 to Sentember 1955.

Flow summary. -1951-55:

Maximum discharge, 6,110 cfs Jan. 22, 1954 (gage height, $18.30 \mathrm{ft}$ )。 Minimum discharge for indicated consecutive period:

\begin{tabular}{rcl} 
Period & Discharge (cfs) & \multicolumn{1}{c}{ Dates } \\
1-day & 23 & Oct. 8, 1954 \\
7-day & 28.9 & Oct。 5-11, 1954 \\
10-day & 29.4 & Oct. 5-14, 1954 \\
20-day & 33.4 & Sept。25-0ct. 14, 1954 \\
30-day & 34.7 & Sept. 15-0ct. 14, 1954 \\
60-day & 45.0 & Aug. 31-Oct. 29, 1954 \\
90-day & 55.5 & Aug. 11-Nov. 8, 1954 \\
120-day & 62.2 & July 20-Nov. 16, 1954 \\
183-day & 72.6 & June 6-Dec. 5, 1954 \\
12-month & 113 & September 1954-August 1955
\end{tabular}

Duration of daily flow

Storage required to mantain indicated flow

\begin{tabular}{cc}
\hline $\begin{array}{c}\text { Percent time } \\
\text { indicated flow was } \\
\text { egialed or exceeded }\end{array}$ & $\begin{array}{c}\text { Discharge } \\
\text { (mgd) }\end{array}$ \\
\hline 20 & 120 \\
30 & 91 \\
50 & 66 \\
70 & 51 \\
80 & 43 \\
90 & 35 \\
95 & 29 \\
98 & 23 \\
99 & 20 \\
99.5 & 18 \\
99.8 & 17 \\
99.9 & 16
\end{tabular}

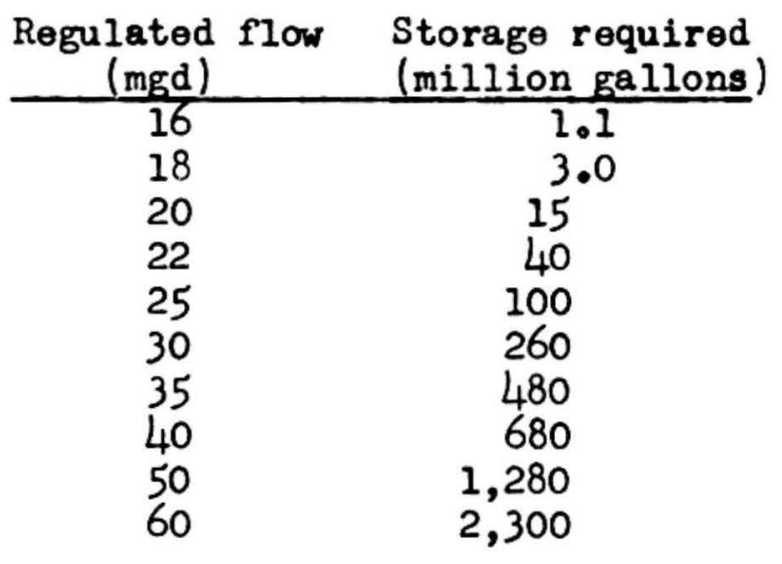

Remarks.-Slight diurnal fluctuation at low flow caused by mills above station. 
South Yadkin River at Cooleeme日

Station No. 61

Location.-Lat $35^{\circ} 48^{\prime}$, long $80^{\circ} 34^{\prime}, 150$ feet downstream from tailrace of Erwin Cotton Mills at Cooleemee, Davie County, 550 ft upstream from bridge on State Highway $801,2-1 / 4$ miles downstream from Bear Creek, and 2-1/2 miles upstream from Third Creek. Datum of gage is $624.57 \mathrm{ft}$ above mean sea level, datum of 1929, supplementary adjustment of 1936.

Drainage area.-569 sq mi.

Records available.-June 1928 to September 1955.

Flow summary $-1928-55$ :

Average discharge (25 years), $606 \mathrm{cfs}$.

Maximum discharge, 24,800 cfs 0ct. 3, 1929 (gage height, 32.25 ft'.

Minimum discharge for indicated consecutive period:

$\begin{array}{cc}\text { Period } & \text { Discharge } \\ \text { 1-day } & 23 \\ \text { 7-day } & 70.4 \\ \text { 10-day } & 76.6 \\ \text { 20-day } & 82.2 \\ \text { 30-day } & 82.2 \\ \text { 60-day } & 1.24 \\ \text { 90-day } & 145 \\ \text { 120-day } & 163 \\ \text { 183-day } & 200.6 \\ \text { 12-month } & 343.1\end{array}$

Duration of daily flow $(\mathrm{cfs})$

Detes
Oct. 12, $191+1$

Oct. 6-12, 1954

Sept。11-20, 1954

Sept。25-Oct. it, 1954

Sept。11-0ct。10, 1954

Aug。 31-0ct. 29, 1954

Aug 。 6-Nov。 3, 1954

July 8-Nov。 4, 1954

May 30-Nov。 28, 1954

April 1954-March 1955

Storage required to maintain indicated flow

\begin{tabular}{cc}
\hline $\begin{array}{c}\text { Percent time } \\
\text { indicated flow was } \\
\text { equaled or exceeded }\end{array}$ & $\begin{array}{c}\text { Discharge } \\
\text { (mgd) }\end{array}$ \\
\hline 20 & 450 \\
30 & 360 \\
50 & 270 \\
70 & 200 \\
80 & 170 \\
90 & 130 \\
95 & 100 \\
98 & 78 \\
99 & 61 \\
99.5 & 52 \\
99.8 & 39 \\
99.9 & 27
\end{tabular}

Remarks.-Large diurnal fluctuation and slight regulation during low and medium flow caused by Erwin Cotton Mill above station. 


\section{Third Creek at Cleveland}

Station No. 62

Location.-Lat $35^{\circ} 45^{\prime}$, long $80^{\circ} 41^{\prime}, 200 \mathrm{ft}$ downstream from highway bridge, three-quarters of a mile north of Cleveland, Rowan County, and 7 miles upstream from Fourth Creek. Datum of gage is $684.47 \mathrm{ft}$ above mean sea level, datum of 1929, supplementary adjustment of 1936 .

Drainage area. $-87.4 \mathrm{sq} \mathrm{mi}$.

Records available.-April 1940 to September 1955.

Flow summary. - 1940-55:

Average discharge ( 15 years), 85.5 cfs.

Maximum discharge, 3,080 cfs about Sept. 19, 1945 (gage height, $15.76 \mathrm{ft}$ ); maximum stage known, $22.5 \mathrm{ft}$, sometime in July 1916.

Minimum discharge for indicated consecutive period:

\begin{tabular}{rcl} 
Period & Discharge (cfs) & \multicolumn{1}{c}{ Dates } \\
1-day & 11 & Sept。 8, 1954 \\
7-day & 11.3 & Sept。 8-14, 1954 \\
10-day & 11.4 & Sept。6-15, 1954 \\
20-day & 12.1 & Aug。 31-Sept。19, 1954 \\
30-day & 18.0 & Sept。18-0ct。7, 1954 \\
60-day & 17.4 & Aug。27-Oct。25, 1954 \\
90-day & 19.6 & July 31-Oct。28, 1954 \\
120-day & 21.5 & July 6-Nov。2, 1954 \\
183-day & 26.6 & June 6-Dec。5, 1954 \\
12-month & 46.4 & April 1954-March 1955
\end{tabular}

Duration of daily flow

Storage required to mintain indicated flow

Percent time indicated flow was equaled or exceeded

\begin{tabular}{lc} 
d or exceeded & mgd) \\
\hline 20 & 61 \\
30 & 50 \\
50 & 37 \\
70 & 28 \\
80 & 23 \\
90 & 19 \\
95 & 16 \\
98 & 13 \\
99 & 11 \\
99.5 & 8.1
\end{tabular}

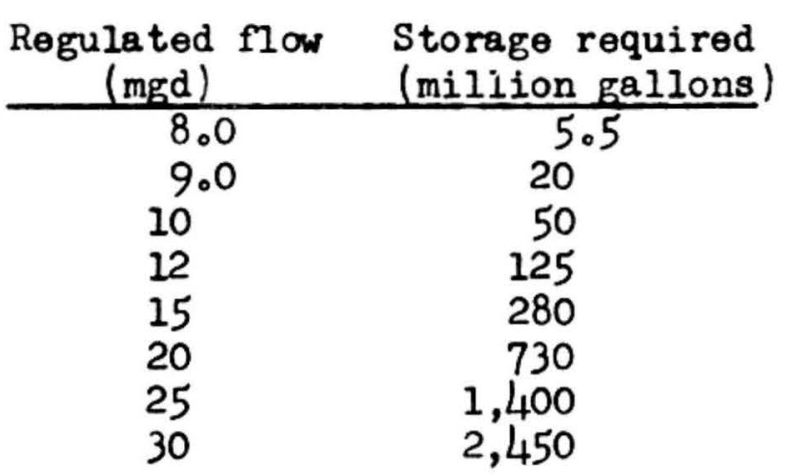

Remarks.-Town of Statesville diverts about three-quarters of a cubic foot per second into basin above station. Creek channel improved considerably by dredging sirce 1916. 


\section{Abbotts Creek at Lexington}

Station No. 63

Location.-Lat $35^{\circ} 48^{\prime} 24^{n}$, long. $80^{\circ} 1_{4^{\prime}}^{\prime} 06^{\prime \prime}$, 200 ft downstroam from small tributary, $200 \mathrm{ft}$ upstream from highway bridge, 0.6 mile downstream from bridge on U. S. Highway $64,1-1 / 2$ miles so utheast of Lexington, Davidson County and 4.9 miles downstream from Rich Fork. Datum of gage is 622.55 ft above mean sea level, datum of 1929, supplementary adjustment of 1936.

Draimage area.-174 sq $\mathrm{mi}$.

Records avai lable.-March 1940 to September 1955.

Flow summary $0-1940-1955$ :

Average discharge (15 years), 161 cfs.

Maximum discharge, 14,800 cfs Sept. 25, 1947 (gage height, $22.12 \mathrm{ft}$ ).

Minimum discharge for indicated consecutivo period:

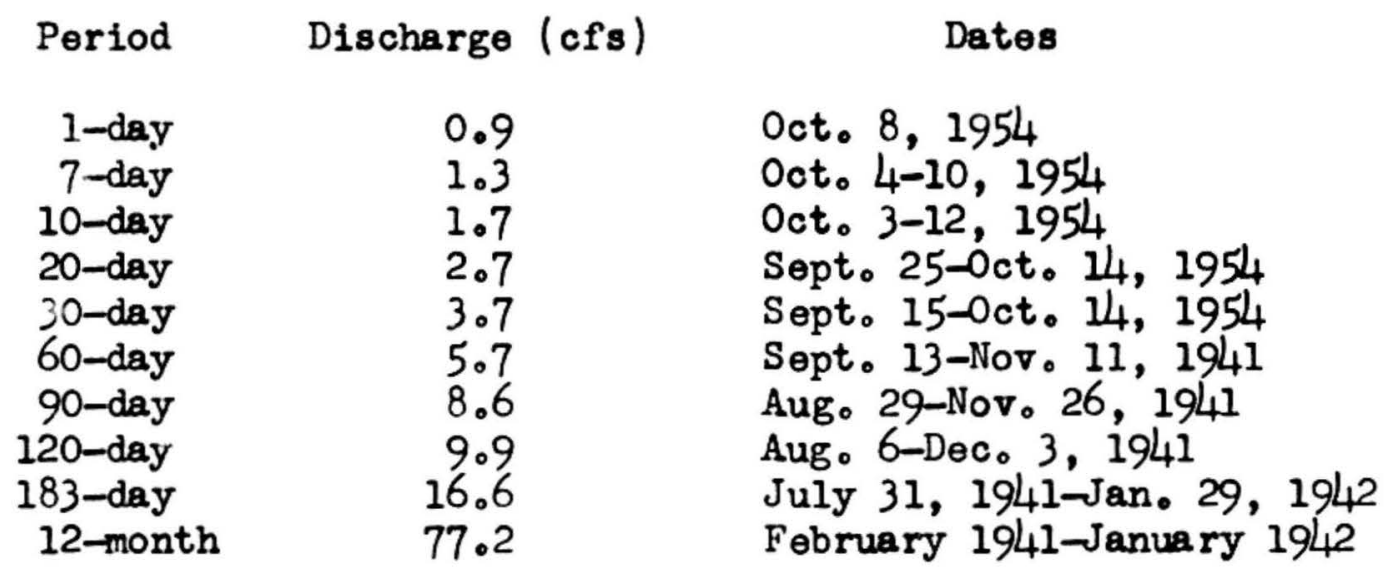

Duration of daily flow

Storage required to maintain indicated flow

\begin{tabular}{cc}
\hline $\begin{array}{c}\text { Percent time } \\
\text { indicated flow ras } \\
\text { equaled or exceeded }\end{array}$ & $\begin{array}{c}\text { Discharge } \\
\text { (mgd) }\end{array}$ \\
\hline 20 & 110 \\
30 & 75 \\
50 & 44 \\
70 & 25 \\
80 & 18 \\
90 & 11 \\
95 & 7.4 \\
98 & 5.0 \\
99 & 3.6 \\
99.5 & 2.8 \\
99.8 & 1.9 \\
99.9 & 1.1
\end{tabular}

Regulated flow Storage required $\frac{(\text { mgd) }}{2.6} \frac{\text { (million gallons) }}{19}$

3.0

4.0

5.0

7.0

12

18

27

42

50
30

70

120

250

700

1,500

3,000

6,000

7,500

Remarks.-Town of Lexington diverted an average of 2.6 cfs for water supply. City of High Point discharges about 3 cfs sewage effluent diverted from Deep River (Cape Fear River basin) into Rich Fork above station. 
Yadkin River at High Rock

Station No。 64

Location.-Lat $35^{\circ} 35^{\prime} 4^{\prime \prime}$, long。 $80^{\circ} 13^{\prime} 59^{\prime \prime}$, on right bank, 0.3 mile downstream from High Rock Dam, 0.6 mile west of High Rock, Davidson County, 1-3/4 miles upstream from Lick Creek, and at mile 253. Datum of gage is 558.68 ft above mean sea level, datum of 1929 , and $590.00 \mathrm{ft}$ above Carolina Aluminum Company datum.

Drainage area. $-3,980 \mathrm{sq} \mathrm{mi}$, approximately.

Records available.-January 1919 to November 1027, November 1941 to September 1955.

Flow summary.-1941-1955:

Average discharge (13 years), 4,481 cfs (19/42-1955).

Maximum di scharge, 71,900 efs Sept. 18, 1945 (gage height, $15.37 \mathrm{ft}$ )。

Maximum stage known, $22.1 \mathrm{ft}$ in July 1916;

Flood of July 21, 1919 reached a stage of $15.9 \mathrm{ft}$ (discharge, 76,600 cfs).

Minimum discharge for indicated consecutive period:

\begin{tabular}{rcl} 
Period & Discharge (cfs) & \multicolumn{1}{c}{ Dates } \\
& & \\
1-day & 12 & Aug。 9, 1942 \\
7-day & 53.6 & Sept。9-15, 1951 \\
10-day & 333 & Aug。6-15, 1954 \\
20-day & 552 & Aug。 5-2l, 1954 \\
30-day & 742 & Aug。 1-30, 1954 \\
60-day & 1,110 & July 31-Sept。 28, 1954 \\
90-day & 1,180 & Aug。9-Nov。 8, 1953 \\
120-day & 1,250 & Aug。9-Dec。6, 1953 \\
12-month & 2,960 & April 1954-March 1955
\end{tabular}

Duration of daily flow

Storage required to maintain indicated flow

\begin{tabular}{cc}
\hline $\begin{array}{c}\text { Percent time } \\
\text { indicated flow was } \\
\text { equaled or exceeded }\end{array}$ & $\begin{array}{c}\text { Discharge } \\
\text { (mgd) }\end{array}$ \\
\hline 20 & 3,600 \\
30 & 3,200 \\
50 & 2,700 \\
70 & 2,000 \\
80 & 1,500 \\
90 & 650 \\
95 & 430 \\
98 & 220 \\
99 & 180 \\
99.5 & 140 \\
99.8 & 110 \\
99.9 & 96
\end{tabular}

\begin{tabular}{cc}
$\begin{array}{c}\text { Regulated flow } \\
\text { (mgd) }\end{array}$ & $\begin{array}{c}\text { Storage required } \\
\text { (million gallons) }\end{array}$ \\
\hline 10 & 3.0 \\
30 & 20 \\
40 & 39 \\
50 & 120 \\
70 & 260 \\
200 & 1,200 \\
500 & 3,400 \\
800 & 10,000 \\
1,000 & 36,000 \\
1,500 & 90,000
\end{tabular}

Remarks.-Except for major floods, flow completely regulated by High Rock Reservoir since 1927 (usual capacity, 10,230,000,000 cubic feet)。 


\section{Uwharrie River near Eldorado}

Station No. 65

Location.-Lat $35^{\circ} 25^{\prime} 30^{\prime \prime}$, long. $80^{\circ} 01^{\prime} 00^{\prime \prime}$, $300 \mathrm{ft}$ downstream from State Highway 109 , one mile upstream from McLeans Creek, and 3 miles south of Eldorado, Montgomery County. Altitude of gage is 29 ? $\mathrm{ft}$.

Drainage area.-347 sq mi。

Records available. - October 1938 to September 1955.

Flow summary. -1938-55:

Average discharge ( 17 years), 318 cfs.

Maximum discharge, 23,300 cfs Sept. 18, 1945 (gage height, $26.22 \mathrm{ft}$ ).

Minimum discharge for indicated consecutive period:

Period Discharge (cfs)

Dates

$\begin{array}{cc}\text { 1-day } & 0.5 \\ 7 \text {-day } & .71 \\ \text { 10-day } & .74 \\ \text { 20-day } & 1.03 \\ \text { 30-day } & 1.52 \\ \text { 60-day } & 2.00 \\ 90-\text { day } & 4.24 \\ \text { 120-day } & 7.22 \\ \text { 183-day } & 16.5 \\ \text { 12-month } & 124_{4}\end{array}$

Oct. 13, 1941

Nov. 5-11, 1941

Nov. 5-14, 1941

Oct. 30-Nov。 18, 1941

Oct. 19-Novo 17, 1941

Sept. 19-Nov。 17, 1941

Sept. 7-Dec。 5, 1947

Aug. 6-Dec. 3, 1941

Aug。 2, 194l-Jan. 31, 1942

February 1941-January 1942

Duration of daily flow

Storage required to maintain indicated flow

\begin{tabular}{cc}
\hline $\begin{array}{c}\text { Percent time } \\
\text { indicated flow was } \\
\text { equaled or exceeded }\end{array}$ & $\begin{array}{c}\text { Discharge } \\
\text { (mgd) }\end{array}$ \\
\hline 20 & 220 \\
30 & 150 \\
50 & 81 \\
70 & 41 \\
80 & 28 \\
90 & 16 \\
95 & 901 \\
98 & 3.7 \\
99 & 1.4
\end{tabular}

\begin{tabular}{cc}
$\begin{array}{c}\text { Regulated flow } \\
\text { (mgd) }\end{array}$ & $\begin{array}{c}\text { Storage required } \\
\text { (million gallons) }\end{array}$ \\
\hline 0.7 & 2.1 \\
1.1 & 8.2 \\
2.2 & 50 \\
2.6 & 78 \\
3.0 & 100 \\
4.5 & 200 \\
7.0 & 400 \\
20 & 2,000 \\
42 & 6,000 \\
80 & 15,500
\end{tabular}

Remarks.- Marked diurnal fluctuation and some regulation for short periods at low flow caused by grist mill above station. Town of Asheboro diverts an average of $1.0 \mathrm{cfs}$ from the basin above the station for water supply. Sewage returns to Deep River (Cape Fear River basin). 


\section{Big Bear Creok near Riohfield}

Station No. 66

Location.-Lat $35^{\circ} 20^{\prime}$, long. $80^{\circ} \mathrm{O}^{\prime}, 200 \mathrm{ft}$ upstream from highway bridge, 300 feet downstream from Little Creek and 10 miles Southwest of Richfield, Stanly County. Altitude of gage is $360 \mathrm{ft}$.

Drainage area. $-56.3 \mathrm{sq} \mathrm{mi}$.

Records available.-May 1954 to September 1955.

Flow summary. -1954-55:

Maximum discharge, 4,120 cfs Feb。6, 1955 (gage height, $11.90 \mathrm{ft}$ ). Minimum discharge for indicated consecutive period:

Period Discharge (cfs) Dates

\begin{tabular}{|c|c|c|}
\hline $\begin{array}{l}\text { 1-day } \\
\text { 7-day } \\
\text { 10-day } \\
\text { 20-day } \\
\text { 30-day } \\
60-\text { day } \\
\text { 90-day } \\
120 \text {-day } \\
183-\text { day }\end{array}$ & $\begin{array}{l}0 \\
0 \\
0 \\
0 \\
0 \\
1.7 \\
7.5 \\
11.6 \\
16.7\end{array}$ & 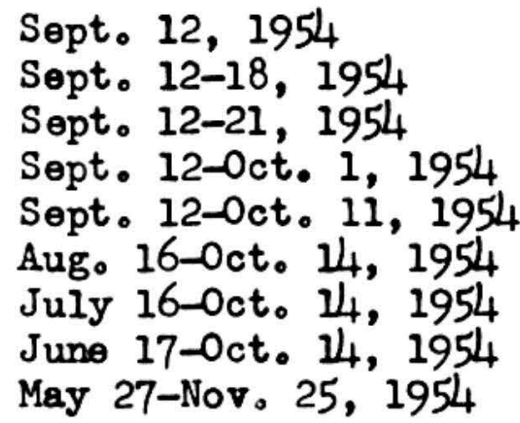 \\
\hline
\end{tabular}

Storege required to mintain indicated flow

\begin{tabular}{cc}
\hline $\begin{array}{c}\text { Regulated flow } \\
\text { (mgd) }\end{array}$ & $\begin{array}{c}\text { Storage required } \\
\text { (million gallons) }\end{array}$ \\
\hline 0.3 & 14 \\
.5 & 20 \\
1.0 & 40 \\
2.0 & 92 \\
3.0 & 160 \\
5.0 & 320 \\
10 & 820 \\
20 & 2,000 \\
30 & 3,500
\end{tabular}




\section{Rocky River near Norwood}

Station No。 67

Location.-Lat $35^{\circ} 09^{\prime} 00^{n}$, long. $80^{\circ} 10^{\prime} 30^{\prime \prime}$, 1,000 ft downstream from Lanes Creek, 1-1/2 miles upstream from highway bridge, and 6 miles southwest of Norwood, Stanly County. Datum of gage is $212.91 \mathrm{ft}$ above mean sea level, Datum of 1929.

Drainage area.-1,370 sq $\mathrm{mi}$, approximately.

Records available.-October 1929 to September 1955.

Flow summary. -1929-55:

Average di scharge (26 years), 1,251 cfs.

Maximum discharge, 155,000 cfs Sept。 18, 1945 (gage height, $46.37 \mathrm{ft}$ ).

Minimum discharge for indicated consecutive period:

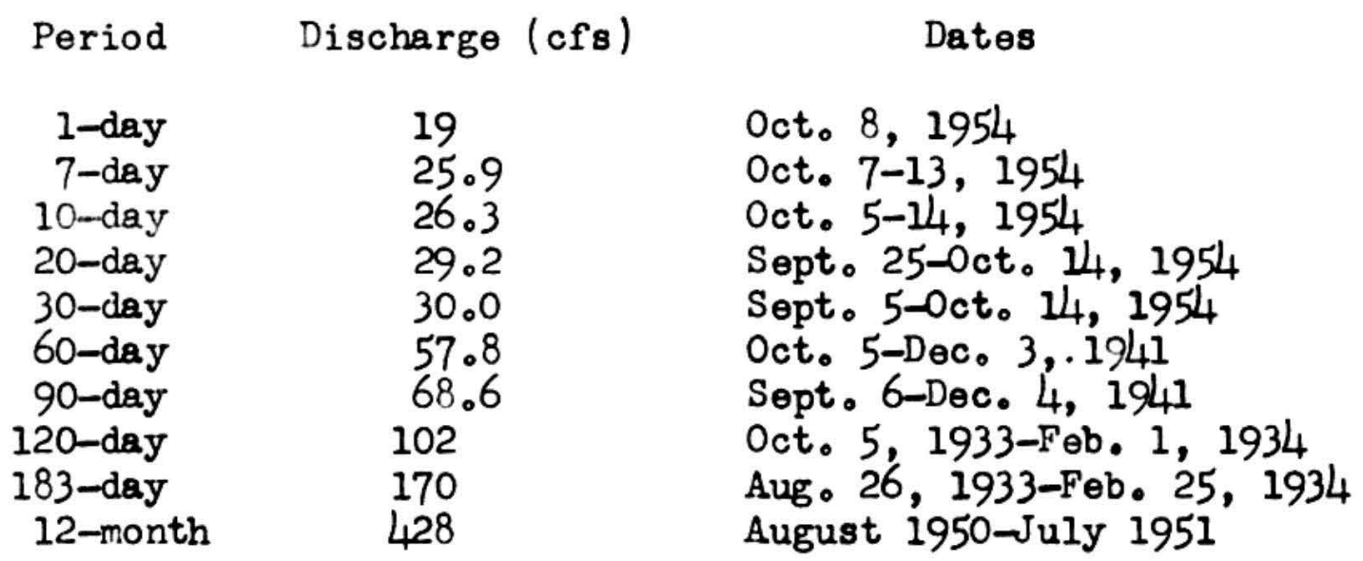

Duration of daily flow

Storage required to maintain indicated flow

\section{Percent time} indicated flow was equaled or exceeded 20 30

50

70

80

90

95

98

99

99.5

99.8

99.9
Discharge (mgd)

810

520

240

120

85

58

44

33

27

22

19

17 


\section{Brown Creek near Polkton}

Station No. 68

Location.-Lat $35^{\circ} 02^{\prime} 10^{\prime \prime}$, long。 $80^{\circ} 08^{\prime} 40^{\prime \prime}$, $400 \mathrm{ft}$ downstream from bridge on State Highway $742,3-1 / 2$ miles downstream from Little Brown Creek, and 4 miles northeast of Polkton, Anson County. Altitude of gage is $216 \mathrm{ft}$.

\section{Drainage area. $-110 \mathrm{sq} \mathrm{mi}$.}

Records available. - October 1937 to September 1955.

Flow summary.-1937-55:

Average discharge ( 18 years), 81.4 cfs.

Maximum discharge, 17,300 cfs Sept. 18, 1945 (gage height, $17.68 \mathrm{ft}$ ).

$$
\text { Period Discharge (cfs) Dates }
$$

\begin{tabular}{|c|c|c|}
\hline $\begin{array}{l}\text { 1-day } \\
7-\text { day } \\
10-\text { day } \\
20 \text {-day } \\
\text { 30-day } \\
60-\text { day } \\
\text { 90-day } \\
120-\text { day } \\
183 \text {-day } \\
12 \text {-month }\end{array}$ & $\begin{array}{l}0 \\
0 \\
0 \\
0 \\
0 \\
.0002 \\
.044 \\
.77 \\
1.26 \\
18.4\end{array}$ & 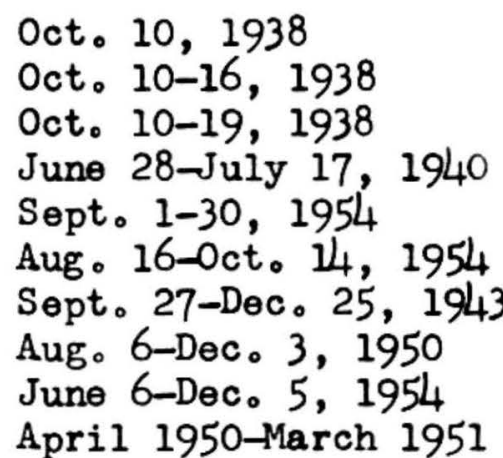 \\
\hline
\end{tabular}

Duration of daily flow

Storage required to maintain indicated flow

\section{Percent time} indicated flow was equaled or exceeded

$\begin{array}{lc}20 & 48 \\ 30 & 22 \\ 50 & 6.1 \\ 70 & 1.3 \\ 80 & .43 \\ 90 & .10\end{array}$

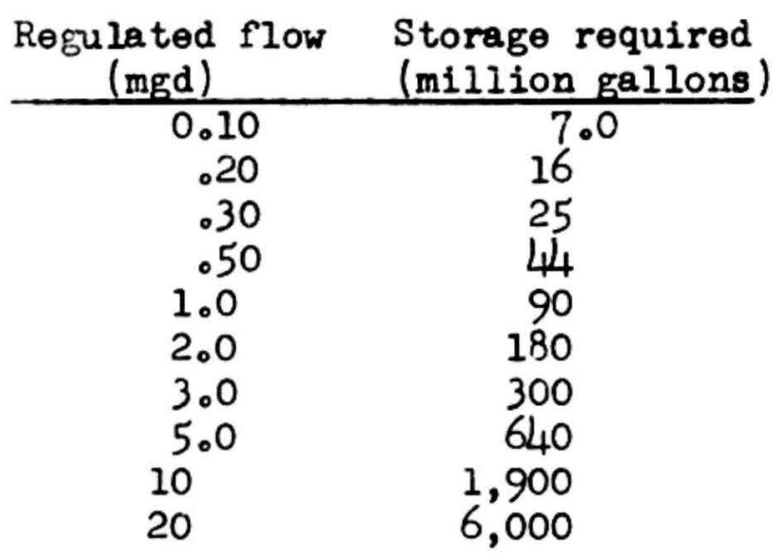




\section{Little River near Star}

Station No. 69

Location.-Lat $35^{\circ} 23^{\prime}$, long. $79^{\circ} 50^{\prime}$, at bridge, a quarter of a mile upstream from Norfolk Southern Railroad bridge, 0.3 mile downstream from Nest Fork Little River, and 3 miles west of Star, Montgomery County. Datum of gage is $409 \mathrm{ft}$ above mean sea level.

Drainage area. $-97.6 \mathrm{sq} \mathrm{mi}$.

Records available.-April 1954 to September 1955.

Flow summary $-1954-55$ :

Maximum discharge, 10,400 cfs 0ct. 15, 1954 (gage height, $16.46 \mathrm{ft}$ ). Flood of September 1948 reached a stage of about $20 \mathrm{ft}$. Minimum discharge for indicated consecutive period:

\begin{tabular}{rcl} 
Period & Discharge (cfs) & \multicolumn{1}{c}{ Dates } \\
1-day & 0.3 & Oct.6, 1954 \\
7-day & .31 & Oct. 8-14, 1954 \\
10-day & .33 & Oct. 5-14, 1954 \\
20-day & 1.28 & Sept. 25-0ct. 14, 1954 \\
30-day & 1.24 & Sept. 15-0ct. 14, 1954 \\
60-day & 7.63 & Aug. 16-0ct. 14, 1954 \\
90-day & 8.45 & July 17-0ct. 14, 1954 \\
120-day & 12.3 & June 17-0ct. 14, 1954 \\
183-day & 56.2 & May 30-Nov. 28, 1954
\end{tabular}

Storage required to mainta in indiceted flow

\begin{tabular}{cc}
\hline $\begin{array}{c}\text { Regulated flow } \\
\text { (mgd) }\end{array}$ & $\begin{array}{c}\text { Storage required } \\
\text { (nillion gallons) }\end{array}$ \\
\hline 0.7 & 4.6 \\
1.0 & 9.6 \\
2.0 & 38 \\
4.0 & 135 \\
7.0 & 330 \\
10 & 560 \\
20 & 1,500 \\
40 & 3,900 \\
60 & 6,600
\end{tabular}




\section{Pee Dee River near Rockingham}

Station No. 70

Location.-Lat $34^{\circ} 56^{\prime} 40^{\prime \prime}$, long. $79^{\circ} 52^{\prime} 10^{\prime \prime}$, at bridge on U.S. Highway 74 , 2.5 miles upstream from Falling Creok, 3.3 miles downstream from Blewett Falls hydroelectric plant, 6 miles west of Rockingham, Richmond County, and at mile 187. Datum of gage is $120.68 \mathrm{ft}$ above mean sea level.

Drainage area. $-6,870$ sq mi approximately。

Records available. - August 1906 to January 1912 (gage heights and fragmentary discharge only), September 1927 to September 1955.

Flow summary. -1927-1955:

Average discharge (28 years), 7,716 ofs (unadjusted).

Maximum discharge, 270,000 cfs Sept。 18, 1945 (gage height, $30.80 \mathrm{ft}$ ).

Maximum stage known, $31.28 \mathrm{ft}$ Aug. 27, 1908 (discharge, 276,000 cfs).

Minimum discharge for indicated consecutive period:

$\begin{array}{rr}\text { Period } & \text { Dischar } \\ \text { 1-day } & 58 \\ \text { 7-day } & 420 \\ \text { 10-day } & 488 \\ 20 \text {-day } & 545 \\ \text { 30-day } & 679 \\ \text { 60-day } & 1,020 \\ 90 \text {-day } & 1,640 \\ \text { 120-day } & 2,020 \\ 183 \text {-day } & 2,640 \\ 12 \text {-month } & 4,080\end{array}$

Duration of daily flow

\section{Dates}

$$
\begin{aligned}
& \text { Dec。2, } 1951 \\
& \text { Sept. 24-30, } 1954 \\
& \text { Sept. 24-Oct. 3, } 1954 \\
& \text { Sept. 24-Oct. 13, } 1954 \\
& \text { Sept. 15-Oct. 14, } 1954 \\
& \text { Aug. 15-Oct. 13, } 1954 \\
& \text { July 19-0ct. 15, } 1954 \\
& \text { June 17-nct. 14, } 1954 \\
& \text { May 27-Nov。 25, } 1951 \\
& \text { April 1933-March } 1934
\end{aligned}
$$

Storage required to maintain indicated flow

\begin{tabular}{cc}
\hline $\begin{array}{c}\text { Percent time } \\
\text { indicated flow was } \\
\text { equaled or exceeded }\end{array}$ & $\begin{array}{c}\text { Discharge } \\
\text { (mgd) }\end{array}$ \\
\hline 20 & 5,900 \\
30 & 4,900 \\
50 & 3,600 \\
70 & 2,800 \\
80 & 2,100 \\
90 & 1,400 \\
95 & 760 \\
98 & 340 \\
99 & 220 \\
99.5 & 160 \\
99.8 & 130 \\
99.9 & 110
\end{tabular}

\begin{tabular}{cc}
$\begin{array}{c}\text { Regulated flow } \\
\text { (mgd) }\end{array}$ & $\begin{array}{c}\text { Storage required } \\
\text { (million }\end{array}$ \\
\hline 85 & 47 \\
150 & 115 \\
300 & 270 \\
400 & 470 \\
500 & 1,800 \\
600 & 6,000 \\
800 & 14,000 \\
1,000 & 24,000 \\
1,500 & 50,000 \\
2,000 & 90,000 \\
2,600 & 170,000
\end{tabular}

Remarks.-Large diurnal fluctuation caused by powerplants above station. Flow largely regulated since 1928 by four reservoirs with a combined usable capacity of $24,259,016,000$ cubic feet. 


\section{Drowning Creek near Hof fman}

Station No. 71

Location.-Lat $35^{\circ} 03^{\prime} 3^{\prime \prime \prime}$, long. $79^{\circ} 29^{\prime} 39^{\prime \prime}$, at bridge on U. S. Highway 1 , three-quarters of a mile downstream from Deep Creek, 1 mile upstream from Seaboard Airline Railway bridge, and 4 miles northeast of Hoffman, Richmond County. Altitude of gage is $270 \mathrm{ft}$.

Drainage area. $-178 \mathrm{sq} \mathrm{mi}$.

Records available. - October 1939 to September 1955.

Flow summary. $-1939-55$ :

Average discharge ( 16 years), $253 \mathrm{cfs}$.

Maximum discharge, 10,900 cfs Sept. 18, 1945 (gage height, 10.29 cfs).

Minimum discharge for indicated conseartive period:

$\begin{array}{cc}\text { Period } & \text { Discharge } \\ & \\ \text { 1-day } & 29 \\ \text { 7-day } & 31.3 \\ \text { 10-day } & 32.6 \\ \text { 20-day } & 38.2 \\ \text { 30-day } & 42.1 \\ \text { 60-day } & 47.2 \\ \text { 90-day } & 64.7 \\ \text { 120-day } & 67.3 \\ \text { 183-day } & 79.9 \\ \text { 12-month } & 137\end{array}$

Duration of daily flow
Dates

Aug. 3, 1940

July 29-Aug. 4, 1940

July 27-Aug. 5, 1940

July 22-Aug. 10, 1940

Sept. 9-0ct. 8, 1954

Sept. 1-Oct. 30, 1940

July 18-Oct. 15,1954

June 28-Oct. 25, 1940

May 14-Nov. 12, 1940

January 1951-December 1951

Storage required to maintain indicated flow

\begin{tabular}{cc}
\hline $\begin{array}{c}\text { Percent time } \\
\text { indicated flow was } \\
\text { equaled or exceeded }\end{array}$ & $\begin{array}{c}\text { Discharge } \\
\text { (mgd) }\end{array}$ \\
\hline 20 & 240 \\
30 & 190 \\
50 & 140 \\
70 & 94 \\
80 & 74 \\
90 & 56 \\
95 & 43 \\
98 & 32 \\
99 & 30 \\
99.5 & 27 \\
99.8 & 25 \\
99.9 & 23
\end{tabular}

Regulated flow Storage required

$\frac{(\mathrm{mgd})}{20} \quad$ (million gallons)

21

5

22

26

29

12

32

47

40

100

50

200

65

540

1,300

90

3,000

7,500 
Lumber River at Boardman

Station No. 72

Location.-Lat $34^{\circ} 26^{\prime}$, long. $78^{\circ} 58^{\prime}$, at bridge on U. S. Highway 74,1 mile downstream from Atlantic Coast Line Railroad bridge at Boardman, Columbus County, and 1-1/2 miles downstream from Big Swamp. Datum of gage is 72.05 ft above mean sea level, datum of 1929.

Drainage area.-1,220 sq $\mathrm{mi}$, approximately.

Records available.-September 1929 to September 1955.

Flow summary.-1929-55:

Average discharge (26 years), 1,206 cfs.

Maximum discharge, 13,400 cfs Sept. 24, 1945 (gage height, $10.64 \mathrm{ft}$ ).

Maximum stage known, $11.8 \mathrm{ft}$ in August 1928 (discharge, 25,000 cfs).

Minimum discharge for indicated consecutive period:

Period Discharge (cfs) Dates

$\begin{array}{cc}\text { 1-day } & 88 \\ \text { 7-day } & 91.6 \\ \text { 10-day } & 93.1 \\ \text { 20-day } & 96.2 \\ \text { 30-day } & 97.4 \\ \text { 60-day } & 109 \\ 90-\text { day } & 145 \\ \text { 120-day } & 168 \\ \text { 183-day } & 261 \\ \text { 12-month } & 391\end{array}$

Oct. 14,1954

Oct. $8-14,1954$

Oct. $5-14,1954$

Sept. 25-0ct. 14, 1954

Sept. 15-Oct. 14, 1954

Aug. 16-0ct。14, 1954

July 19-0ct。16, 1954

June 24-Oct。21, 1954

June 5-Dec. 4, 1954

June 1933-May 1934

Duration of daily flow

Storage required to maintain indicated flow

\begin{tabular}{cc}
\hline $\begin{array}{c}\text { Percent time } \\
\text { indicated flow was } \\
\text { equaled or exceeded }\end{array}$ & $\begin{array}{c}\text { Discharge } \\
\text { (mgd) }\end{array}$ \\
\hline 20 & 1,100 \\
30 & 870 \\
50 & 590 \\
70 & 340 \\
80 & 260 \\
90 & 180 \\
95 & 140 \\
98 & 110 \\
99 & 92 \\
99.5 & 78 \\
99.8 & 60
\end{tabular}

\begin{tabular}{cc}
$\begin{array}{c}\text { Regulated flow } \\
\text { (mgd) }\end{array}$ & $\begin{array}{c}\text { Storage required } \\
\text { (million gallons) }\end{array}$ \\
\hline 64 & 50 \\
70 & 150 \\
80 & 600 \\
90 & 1,200 \\
100 & 1,800 \\
150 & 5,500 \\
200 & 11,000 \\
300 & 28,000
\end{tabular}


Catawba River near Marion

Station No. $7^{\text {? }}$

Location.-Lat $35^{\circ} 42^{\prime} 2^{\prime \prime}$, long. $82^{\circ} 02^{\prime} 10^{\prime \prime}$, at bridge on U. S. Highway 221 , 0.2 mile downstream from Tom Creek, and 2.2 miles northwest of Marion, McDowell County. Records include flow of small tributary which enters above control. Datum of gage is 1,208 ft above mean sea level, datum of 1929, supplementary adjustment of 1936.

Drainage area.-171 sq $\mathrm{mi}$ (including area of small tributary which enters above control).

Records available. - October 1941 to September 1955.

Flow summary.-1941-55:

Average discharge ( $y_{4}$ years), 307 cfs.

Maximum di scharge, 19,700 cfs Aug。 28, 1949 (gage height, $15.02 \mathrm{ft}$ ).

Flood of Aug. 13, 1940 reached a stage of $19.34 \mathrm{ft}$ (discharge, $71,400 \mathrm{cfs}$ ).

Minimum discharge for indicated consecutive period:

\begin{tabular}{rcl} 
Period & Discharge (cfs) & \multicolumn{1}{c}{ Dates } \\
& & \\
1-day & 29 & Sept. 30, 1954 \\
7-day & 31.6 & Oct. 9-15, 1954 \\
10-day & 35.3 & Oct. 9-18, 1954 \\
20-day & 37.9 & Oct. 4-23, 1954 \\
30-day & 39.5 & Sept。16-0ct. 15, 1954 \\
60-day & 39.4 & Sept. 2-Oct. 31, 1954 \\
9 -day & 45.8 & Aug。7-Nov。 4, 1954 \\
120-day & 52.0 & July 19-Nov。15, 1954 \\
183-day & 67.2 & June 28-Dec。27, 1954 \\
12-month & 144 & April 1954-March 1955
\end{tabular}

Duration of daily flow

Storage required to maintain indicated flow

\begin{tabular}{cc}
\hline $\begin{array}{c}\text { Percent time } \\
\text { indicated flow was } \\
\text { equaled or exceeded }\end{array}$ & $\begin{array}{c}\text { Discharge } \\
\text { (mgd) }\end{array}$ \\
\hline 20 & 260 \\
30 & 210 \\
50 & 150 \\
70 & 110 \\
80 & 87 \\
90 & 65 \\
95 & 49 \\
98 & 35 \\
99 & 28 \\
99.5 & 25 \\
99.8 & 21 \\
99.9 & 20
\end{tabular}

Regulated flow Storage required

$\frac{(\mathrm{mgd})}{20} \quad$ (million gallons)

25

30

40

50

60

70

80

90

100
51

250

1,100

2,300

3,900

5,600

7,500

9,500

11,000

Remarks.-Considerable diurnal fluctuation and slight regulation at low flow caused by powerplants above station. 
Station No. 74

Location. - Lat $35^{\circ} 47^{\prime} 50^{\prime \prime}$, long $81^{\circ} 53^{\prime} 20^{\prime \prime}$, at bridge on State Highway 126 at Branch, Burke County, and 0.2 mile upstream from Lake James. Datum of gage is $1,205.87 \mathrm{ft}$ above mean sea level, datum of 1929, supplementary adjustment of 1936 .

Drainage area. -65 sq mi, approximately.

Records available.-May 1907 to December 1908 (fragmentary); June 1922 to September 1955 .

Flow summary. - 1907-08, 1922-55:

Average discharge ( 33 years, 1922-55), 139 cfs.

Maximum discharge, 39,500 cfs Aug. 13, 1940 (gage height, $11.4 \mathrm{ft}$ ).

Minimum discharge for indicated consecutive period:

\begin{tabular}{rcl} 
Period & Discharge (cfs) & \multicolumn{1}{c}{ Dates } \\
1-day & 8 & Sept. 7, 1925 \\
7-day & 10.4 & Aug. 22-28, 1925 \\
10-day & 11.3 & Aug. 22-31, 1925 \\
20-day & 11.8 & Aug. 22-Sept. 10, 1925 \\
30-day & 13.0 & Aug. 15-Sept. 13, 1925 \\
60-day & 14.0 & Aug. 15-0ct. 13, 1925 \\
90-day & 16.8 & July 27-Oct. 24, 1925 \\
120-day & 23.8 & July 12-Nov. 8, 1925 \\
183-day & 32.7 & June 29-Dec. 28, 1954 \\
12-month & 66.3 & April 1930-March 1931
\end{tabular}

Duration of daily flow

Storage required to maintain indicated flow

\begin{tabular}{cc}
\hline $\begin{array}{c}\text { Percent time } \\
\text { indicated flow was } \\
\text { equaled or exceeded }\end{array}$ & $\begin{array}{c}\text { Discharge } \\
\text { (mgd) }\end{array}$ \\
\hline 20 & 110 \\
30 & 86 \\
50 & 61 \\
70 & 41 \\
80 & 32 \\
90 & 23 \\
95 & 17 \\
98 & 13 \\
99 & 11 \\
9 & .5 \\
99.8 & 9.1 \\
99.9 & 7.4 \\
\end{tabular}

\begin{tabular}{cc}
$\begin{array}{c}\text { Regulated flow } \\
\text { (mgd) }\end{array}$ & $\begin{array}{c}\text { Storage required } \\
\text { (million gallons) }\end{array}$ \\
\hline 6.0 & 0.85 \\
7.0 & 3.9 \\
8.0 & 12 \\
10 & 60 \\
12 & 180 \\
15 & 410 \\
20 & 870 \\
25 & 1,400 \\
35 & 2,800 \\
45 & 4,500
\end{tabular}

Remarks.-Occasional slight diurnal fluctuation caused by small mill above station. 
Lower Little River near All Healing Springs

Station No。 75

Location.-Lat $35^{\circ} 57^{\prime}$, long. $81^{\circ} \mathrm{L}^{\prime}, 0.3 \mathrm{mile}$ downstream from Grassy Creek, 0.4 mile upstream from Lambert Creek, 2.2 miles northeast of All Healing Springs, Alexander County, and 4 miles northwest of Taylorsville.

Drainage area. $-31.2 \mathrm{sq} \mathrm{mi}$.

Records available.-January 1953 to September 1955.

Flow summary.-1953-55:

Maximum discharge, 1,040 cfs Jan, 22, 1954 (gage height, $11.80 \mathrm{cfs}$ ). Minimum discharge for indicated consecutive period:

\begin{tabular}{rcl} 
Period & Discharge (cfs) & \multicolumn{1}{c}{ Dates } \\
1-day & 3.1 & Sept.29, 1955 \\
7-day & 3.39 & Sept. 17-23, 1955 \\
10-day & 3.74 & Sept. 15-24, 1955 \\
20-day & 4.29 & Sept.21-0ct. 10, 1954 \\
30-day & 4.17 & Sept.9-0ct. 8, 1954 \\
60-day & 5.29 & Aug.30-Oct. 26, 1954 \\
90-day & 6.15 & July 29-Oct.26, 1954 \\
120-day & 6.83 & July 5-Nov。1, 1954 \\
183-day & 9.51 & June 6-Dec。 5, 1954 \\
12-month & 16.4 & April 1954-March 1955
\end{tabular}

Storage required to maintain indicated flow

\begin{tabular}{cc}
\hline $\begin{array}{c}\text { Regulated flow } \\
\text { (mgd) }\end{array}$ & $\begin{array}{c}\text { Storage required } \\
\text { (million gallons) }\end{array}$ \\
\hline 2.3 & 0.5 \\
3.0 & 10 \\
4.0 & 46 \\
5.0 & 106 \\
6.0 & 185 \\
8.0 & 410 \\
10 & 730
\end{tabular}


Catawba River at Catawba

Station No. 76

Location.-Lat $35^{\circ} 43^{\prime}$, long. $81^{\circ} 04^{\prime}$, at bridge on U. S. Highways 64 and 70 , half a mile upstream from Lyle Creek, five-eights of a mile upstream from Southern Railway bridge, and 1 mile northeast of Catawba, Catawba County. Records include flow of Lyle Creek. Datum of gage is $746.49 \mathrm{ft}$ above mean sea level.

Drainage area.- - 1,535 sq $\mathrm{mi}$, includes that of Lyle Creek.

Records available.-July 1896 to April 1902 (gage heights only, 1900, 1902); November 1934 to September 1955.

Flow summary.-1896-1902; 1934-55:

Average discharge (23 years, 1896-99, 1935-55), 2,276 cfs (unadjusted). Maximum discharge, 177,000 cfs Aug. $\mathbb{H}_{4}, 1940$ (gage height, $36.8 \mathrm{ft}$ ).

Maximum stage known, $4.1 \mathrm{ft} J u l y ~ 16,1916$, affected by failure of earth dike 4 miles above station. Discharge not determined. Yinimum discharge for indicated consecutive period:

$\begin{array}{rr}\text { Period } & \text { Dischar } \\ \text { 1-day } & 101 \\ \text { 7-day } & 166 \\ \text { 10-day } & 170 \\ \text { 20-day } & 223 \\ \text { 30-day } & 314 \\ \text { 60-day } & 502 \\ \text { 90-day } & 598 \\ \text { 120-day } & 692 \\ \text { 183-day } & 800 \\ \text { 12-month } & 1,100\end{array}$

Duration of daily flow
Dates

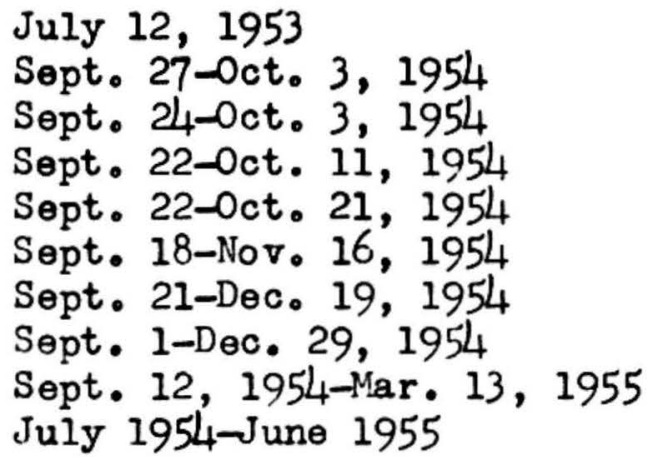

July 12, 1953

Sept. 27-0ct。 3, 1954

Sopt. 24-0ct. 3, 1954

Sept. 22-0ct. 11, 1954

Sept. 22-0ct.21, 1954

Sept. 18-Nov. 16, 1954

Sept. 21-Dec. 19, 1954

Sept. 1-Dec. 29, 1954

July 1954-June 1955

Storage required to maintain indicated flow

\begin{tabular}{cc}
\hline $\begin{array}{c}\text { Percent time } \\
\text { indicated flow was } \\
\text { exualed or exceeded }\end{array}$ & $\begin{array}{c}\text { Discharge } \\
\text { (: igd) }\end{array}$ \\
\hline 20 & 2,000 \\
30 & 1,700 \\
50 & 1,200 \\
70 & 790 \\
80 & 480 \\
90 & 170 \\
95 & 110 \\
98 & 95 \\
99 & 88 \\
99.5 & 81
\end{tabular}

\begin{tabular}{cc}
$\begin{array}{c}\text { Regulated flow } \\
\text { (mgd) }\end{array}$ & $\begin{array}{c}\text { Storage required } \\
\text { (million gallons) }\end{array}$ \\
\hline 80 & 18 \\
90 & 42 \\
100 & 84 \\
150 & 530 \\
200 & 1,300 \\
300 & 3,100 \\
400 & 5,900 \\
500 & 12,000 \\
600 & 20,000 \\
800 & 52,000
\end{tabular}

Remarks.-Flow regulated by four reservoirs above station which have a combined usable capacity of $14,975,000,000$ cu ft. 
Henry Fork near Henry River

Station No。 77

Location.-Lat $35^{\circ} 41^{\prime}$, long。 $81^{\circ} 24^{\prime}$, at old Link Ford, $450 \mathrm{ft}$ downstream from highway bridge, and 2 miles southeast of Henry River, Burke County. Datum of gage is $890.99 \mathrm{ft}$ above mean sea level.

Drainage area.-80 sq mi, approximately.

Records available - July 1925 to November 1931, December 1941 to September 1955.

Flow summary. -1925-31, 1942-55:

Average discharge (19 years), 122 cfs.

Maximum discherge, 15,300 cfs Oct. 2, 1929 (gage height, $18.40 \mathrm{ft}$ ). Maximum stage known, $29.2 \mathrm{ft}$ Aug. 13, 1940 (discharge, 31,300 cfs)。 Minimum discharge for indicated consecutive period:

Period Discharge (cfs)

$\begin{array}{cc}\text { 1-day } & 4 \\ \text { 7-day } & 13.8 \\ \text { 10-day } & 13.3 \\ \text { 20-day } & 21.5 \\ \text { 30-day } & 21.4 \\ \text { 60-day } & 25.4 \\ \text { 90-day } & 24.6 \\ 120 \text {-day } & 32.9 \\ 183 \text {-day } & 36.0 \\ \text { 12-month } & 57.1\end{array}$

Duration of daily flow
Dates

Nov. 15, 1942 July $18-24,1926$ July $17-26,1926$ Sept. 25-0ct。14, 1926 Sept. 17-0ct。16, 1926 Sept. 3-Nov。1, 1926 A.ug . 19-Nov。 15, 1926 July 18-Nov。14, 1354 May 27-Nov。25, 1926 June 1926-May 1927

Storage required to maintain indicated flow

\begin{tabular}{cc}
\hline $\begin{array}{c}\text { Percent time } \\
\text { indicated flow was } \\
\text { equaled or exceeded }\end{array}$ & $\begin{array}{c}\text { Discharge } \\
\text { (mgd) }\end{array}$ \\
\hline 20 & 91 \\
30 & 73 \\
50 & 54 \\
70 & 40 \\
80 & 32 \\
90 & 23 \\
95 & 16 \\
98 & 6.5 \\
99 & 5.0
\end{tabular}

\begin{tabular}{cc}
$\begin{array}{c}\text { Regulated flow } \\
\text { (mgd) }\end{array}$ & $\begin{array}{c}\text { Storage required } \\
\text { (millior. gallons) }\end{array}$ \\
\hline 3.0 & 0.36 \\
4.0 & 1.4 \\
5.0 & 2.5 \\
7.0 & 4.6 \\
9.0 & 7.1 \\
11 & 22 \\
20 & 270 \\
30 & 1,400 \\
40 & 4,100
\end{tabular}

Remarks.-Considerable diurnal fluctuation and some reguls.tion caused by mill above station. City of Morganton diverts an average of 3 cfs from basin for municipal water supply. 
Location.-Lat $35^{\circ} 25^{\prime} 20^{\prime \prime}$, long. $81^{\circ} 15^{\prime} 50^{\prime \prime}$, half a mile upstream from highway bridge, 1-1/2 miles upstream from mouth, 1-1/2 miles south of Laboratory, Lincoln County, and 3-1/2 miles south of Lincolnton. Altitude of gage is $736 \mathrm{ft}$.

Drainage area. $-68.4 \mathrm{sq} \mathrm{mi}$.

Records available.-August 1951 to September 1955.

Flow summary.-1951-55:

Maximum discharge, 5,030 cfs Mar. 4, 1952 (gage height, 8.74 ft). Minimum discharge for indicated consecutive period:

\begin{tabular}{rcl} 
Period & Discharge (cfs) & \multicolumn{1}{c}{ Dates } \\
1-day & 5.2 & Oct. 7, 1954 \\
7-day & 5.95 & Oct. 3-9, 1954 \\
10-day & 6.20 & Oct. 2-11, 1954 \\
20-day & 6.90 & Sept.25-0ct. 14, 1954 \\
30-day & 7.88 & Sept.23-0ct. 22, 1954 \\
60-day & 8.36 & Aug。31-0ct.29, 1954 \\
90-day & 10.2 & Aug. 4-Nov. 1, 1954 \\
120-day & 11.9 & July 19-Nov。 15, 1954 \\
183-day & 16.7 & June 26-Dec.25, 1954 \\
12-month & 35.8 & April 1954-March 1955
\end{tabular}

Duration of daily flow

Storage required to maintain indicated flow

\begin{tabular}{cc}
\hline $\begin{array}{c}\text { Percent time } \\
\text { indicated flow was } \\
\text { equaled or exceeded }\end{array}$ & $\begin{array}{c}\text { Discharge } \\
\text { (mgd) }\end{array}$ \\
\hline 20 & 49 \\
30 & 40 \\
50 & 26 \\
70 & 19 \\
80 & 14 \\
90 & 11 \\
95 & 7.3 \\
98 & 5.0 \\
99 & 4.6 \\
99.5 & 4.2
\end{tabular}

\begin{tabular}{cc}
$\begin{array}{c}\text { Regulated flow } \\
\text { (mgd) }\end{array}$ & $\begin{array}{c}\text { Storage required } \\
\text { (million gallons) }\end{array}$ \\
\hline 4.0 & 1.0 \\
5.0 & 14 \\
6.0 & 50 \\
7.0 & 100 \\
8.0 & 160 \\
9.0 & 240 \\
10 & 330 \\
15 & 970 \\
20 & 1,800 \\
25 & 2,800
\end{tabular}




\section{Long Creek near Bessemer City}

Station No。 79

Location.-Lat $35^{\circ} 18^{\prime} 20^{\prime \prime}$, long. $81^{\circ} y^{\prime} 05^{\prime \prime}$, at highway bridge, 2 miles northeast of Bessemer City, Gaston County, and 8-1/4 miles upstream from mouth. Datum of gage is $706.1 \mathrm{ft}$ above mean sea level, datum of 1929.

Drainage area.-3l.4 sq $\mathrm{mi}$.

Records available.-December 1952 to September 1955.

Flow summary.-1952-55:

Maximum discharge, 1,040 cfs May 21, 1955 (gage height, $5.33 \mathrm{ft}$ )。

Minimu discharge for indicated consecutive period:

\begin{tabular}{|c|c|c|}
\hline Period & Discharge (cfs) & Dates \\
\hline $\begin{array}{l}\text { 1-day } \\
7 \text {-day } \\
\text { 10-day } \\
20 \text {-day } \\
\text { 30-day } \\
60-\text { day } \\
90-\text { day } \\
120 \text {-day } \\
183 \text {-day } \\
\text { 12-month }\end{array}$ & $\begin{array}{l}0.8 \\
1.27 \\
1.39 \\
1.70 \\
1.77 \\
2.16 \\
3.06 \\
3.59 \\
5.30 \\
4.7\end{array}$ & 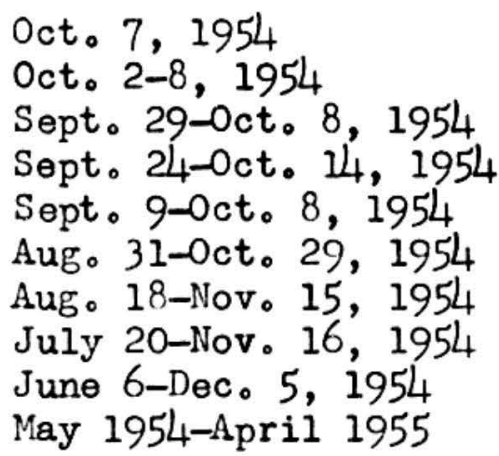 \\
\hline
\end{tabular}

Duration of daily flow

Storage required to maintain indicated flow

\begin{tabular}{cc}
\hline $\begin{array}{c}\text { Percent time } \\
\text { indicated flow was } \\
\text { equaled or exceeded }\end{array}$ & $\begin{array}{c}\text { Discharge } \\
\text { (mgd) }\end{array}$ \\
\hline 20 & $\frac{14}{11}$ \\
30 & 7.0 \\
50 & 4.1 \\
70 & 2.9 \\
80 & 1.8 \\
90 & 1.2 \\
95 & .86 \\
98 & .73 \\
99 & .64 \\
99.5 & .60 \\
99.8 &
\end{tabular}

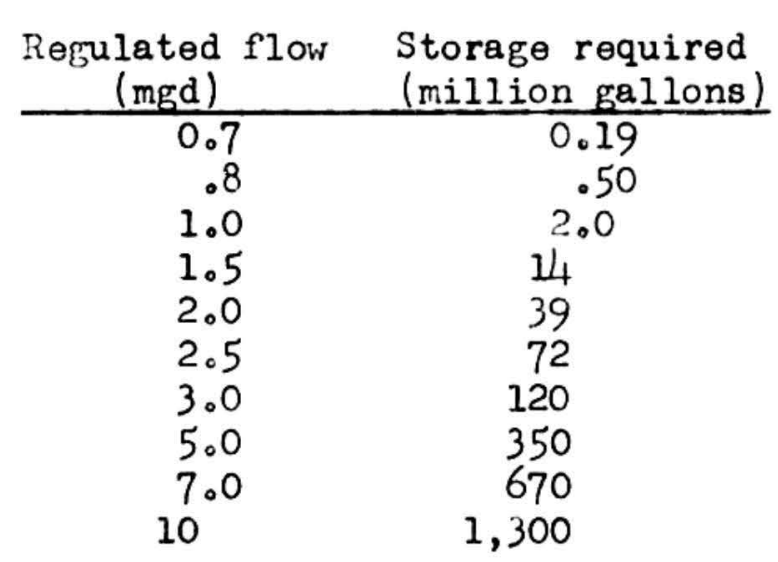

Remarks.-Bessemer City diverts approximately 1.2 cfs out of basin for municipal supply. 


\section{South Fork Catawba River at Lowell}

Station No. 80

Location.-Lat $35^{\circ} 17^{\prime} 09^{\prime \prime}$, long. $81^{\circ} 06^{\prime} 0^{\prime \prime}, 120 \mathrm{ft}$ downstream from Housers Creek, 1 mile north of Lowell, Gaston County, and 3 miles downstream from Long Creek. Datum of gage is $603.10 \mathrm{ft}$ above mean sea level, datum of 1929, supplementary adjustment of 1936.

Drainage area. $-630 \mathrm{sq} \mathrm{mi}$.

Records available.-January 1942 to September 1955.

Flow summary - - 1942-55:

Average discharge (13 ycars), 771 cfs.

Maximum discharge, 22,000 cfs Sept. 19, 1945 (gage height, $16.98 \mathrm{ft}$ ). Maximum stage known, $21.33 \mathrm{ft}$ August 1940 (discharge, 34,000 cfs). Minimum discharge for indicated consecutive period:

$\begin{array}{cc}\text { Period } & \text { Discharge } \\ \text { 1-day } & 31 \\ \text { 7-day } & 76.9 \\ \text { 10-day } & 75.4 \\ \text { 20-day } & 86.8 \\ \text { 30-day } & 93.2 \\ \text { 60-day } & 106 \\ 90 \text {-day } & 138 \\ \text { 120-day } & 152 \\ 183 \text {-day } & 196 \\ \text { 12-month } & 472\end{array}$

Dates

Duration of daily flow

Storage required to maintain indicated flow

\begin{tabular}{cc}
\hline $\begin{array}{c}\text { Fercent time } \\
\text { indicated flow was } \\
\text { equaled or exceeded }\end{array}$ & $\begin{array}{c}\text { Discharge } \\
\text { (mgd) }\end{array}$ \\
\hline 20 & 580 \\
30 & 470 \\
50 & 360 \\
70 & 260 \\
80 & 210 \\
90 & 170 \\
95 & 130 \\
98 & 94 \\
99 & 76 \\
99.5 & 64 \\
99.8 & 38
\end{tabular}

\begin{tabular}{cc}
$\begin{array}{r}\text { Regulated flow } \\
\text { (mgd) }\end{array}$ & $\begin{array}{c}\text { Storage required } \\
\text { (million gallons) }\end{array}$ \\
\hline 26 & 6.0 \\
30 & 10 \\
40 & 21 \\
50 & 31 \\
55 & 62 \\
60 & 130 \\
80 & 760 \\
100 & 2,000 \\
150 & 6,400 \\
300 & 35,000
\end{tabular}

Remarks.-Considerable diurnal fluctuation and slight regulation at low flow caused by powerplant above station. Diversion of approximately $11 \mathrm{cfs}$ are made above station for municipal supplies at cities of Gastonia, Morganton, and Bessemer City. Approximately $2 \mathrm{cfs}$ are returned to basin above station as sewage. 


\section{Little Sugar Creek near Charlotte}

Station No. 81

Location.-Lat $35^{\circ} 09^{\prime} 2^{\prime \prime}$, long. $80^{\circ} 51^{\prime} 10^{\prime \prime}$, $500 \mathrm{ft}$ downstream from Briar Creek, $600 \mathrm{ft}$ upstream from city of Charlotte sewage-disposal plant, and 4.7 miles south of city hall, Charlotte, Mecklenburg County. Datum of gage is 571.6 ft above mean sea level.

\section{Drainage ar a. -41.4 sq $\mathrm{mi}$.}

Records available.-July 1924 to September 1955.

Flow summary.-1924-55:

Average discharge ( 31 years), 44.2 cfs.

Maximum discharge, 8,370 cfs Apr . 6, 1936 (gage height, $16.2 \mathrm{ft}$ ).

Minimum discharge for indicated consecutive period:

\begin{tabular}{|c|c|c|}
\hline Period & Discharge (cfs) & Dates \\
\hline $\begin{array}{l}\text { 1-day } \\
\text { 7-day } \\
10-\text { day } \\
\text { 20-day } \\
\text { 30-day } \\
\text { 60-day } \\
\text { 90-day } \\
120-\text { day } \\
183 \text {-day } \\
\text { 12-month }\end{array}$ & $\begin{array}{l}1.6 \\
2.09 \\
2.12 \\
2.19 \\
2.3 \\
3.28 \\
5.68 \\
7.50 \\
10.0 \\
20.1\end{array}$ & $\begin{array}{l}\text { Aug. 1, 1925 } \\
\text { Oct. 5-11, 1925 } \\
\text { Oct. 4-13, 1925 } \\
\text { Sept. 23-Oct. 12, } 1925 \\
\text { Sept. 14-Oct. 13, 1925 } \\
\text { Aug. 25-Oct. 23, 1925 } \\
\text { Sept. 1-Nov. 29, 1926 } \\
\text { Aug. 27-Dec. 24, 1926 } \\
\text { May 3-Nov。 1, 1926 } \\
\text { September 1926-August } 1927\end{array}$ \\
\hline
\end{tabular}

Duration of daily flow

\begin{tabular}{cc}
\hline $\begin{array}{c}\text { Percent time } \\
\text { indicated flow was } \\
\text { equal od or exceeded }\end{array}$ & $\begin{array}{c}\text { Discharge } \\
\text { (mgd) }\end{array}$ \\
\hline 20 & 27.0 \\
30 & 19 \\
50 & 12 \\
70 & 7.6 \\
80 & 6.0 \\
90 & 4.6 \\
95 & 3.7 \\
98 & 2.9 \\
99 & 2.3 \\
99.5 & 1.8 \\
99.8 & 1.5 \\
99.9 & 1.4
\end{tabular}

Storage required to maintain indicated flow

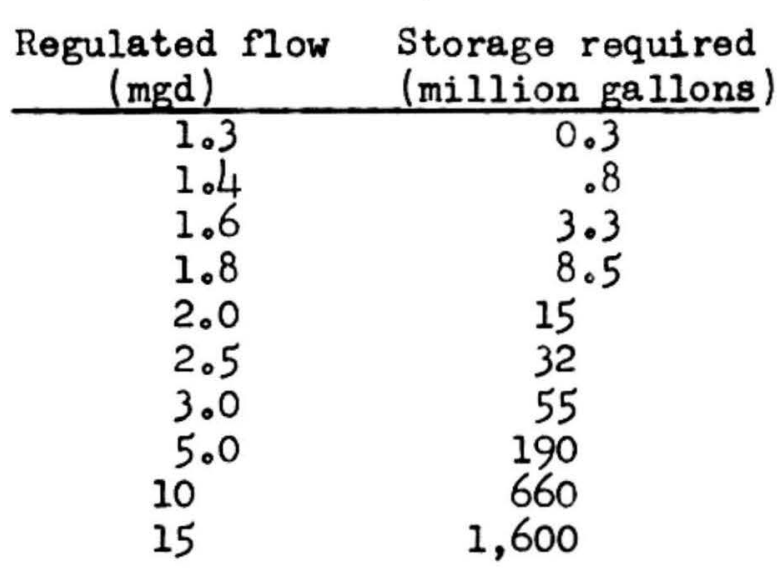




\section{Broad River near Chimney Rock}

Station No。 82

Location.-Lat $35^{\circ} 25^{\prime} 35^{\prime \prime}$, long. $82^{\circ} 10^{\prime} 45^{\prime \prime}$, 1,000 ft downstream from Lake Lure Dam, 1.5 miles downstream from Buffalo Creek, and 3 miles east of Chimney Rock, Rutherford County. Alıitude of gage is $860 \mathrm{ft}$.

Drainage area.-97 sq $\mathrm{mi}$, approximately.

Records available.-May 1907 to June 1909 (fragmentary), March 1927 to September 1955.

Flow summary. -1927-55:

Average discharge (28 years), $171 \mathrm{cfs}$.

Maximum discharge, 26,000 cfs Aug. 15, 1928 (gage height, $16.8 \mathrm{ft}$ )。

Minimum discharge for indicated consecutive period:

\begin{tabular}{ccl} 
Period & Discharge (cfs) & \multicolumn{1}{c}{ Dates } \\
1-day & 0.8 & Sept.24, 1928 \\
7-day & 087 & Sept.23-29, 1928 \\
10-day & .90 & Sept。20-29, 1928 \\
20-day & 11.6 & Oct。9-Oct。28, 1941 \\
30-day & 20.8 & Sept。24-Oct。23, 1954 \\
60-day & 23.4 & Sept。20-Nov。18, 1954 \\
90-day & 31.2 & Aug。17-Nov。14, 1954 \\
120-day & 33.7 & July 22-Nov. 18, 1954 \\
183-day & 43.0 & June 30-Dec。29, 1954 \\
12-month & 76.4 & May 1954-April 1955
\end{tabular}

Duration of daily flow

Storage required to maintain indicated flow

\begin{tabular}{cc}
\hline $\begin{array}{c}\text { Percent time } \\
\text { indicated flow was } \\
\text { equaled or exceeded }\end{array}$ & $\begin{array}{c}\text { Discharge } \\
\text { (mgd) }\end{array}$ \\
\hline 20 & 160 \\
30 & 120 \\
50 & 94 \\
70 & 54 \\
80 & 45 \\
90 & 4.5 \\
95 & 2.6 \\
98 & 1.7 \\
99 & 1.3 \\
99.5 & .97 \\
99.8 & .71 \\
99.9 & .65
\end{tabular}

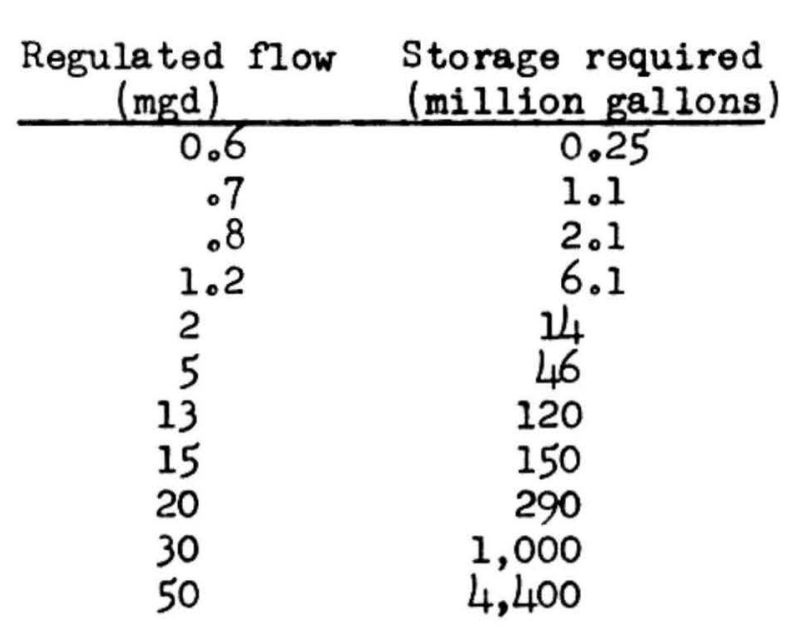

Remarks.-Large diurnal fluctuation and complete regrlation at low flow caused by powerplant above station. 


\section{Cove Creek near Lake Lure}

Station No. 83

Location.-Lat $35^{\circ} 25^{\prime} 30^{\prime \prime}$, long. $82^{\circ} 06^{\prime} 35^{\prime \prime}$, at bridge on U. S. Highways 64 and 74 , I mile upstream from mouth and 5 miles east of Lake Lure, Rutherford County.

Drainage area. $-77.0 \mathrm{sq} \mathrm{mi}$.

Records available.-January 1951 to September 1955.

Flow summary. -1951-55:

Maximum discharge, 7,000 cfs Mar. 11, 1952 (gage height, 14.00 ft). Minimum discharge for indicated consecutive period:

Period

$\begin{array}{ll}\text { 1-day } & 21 \\ \text { 7-day } & 21.2 \\ \text { 10-day } & 21.2 \\ \text { 20-day } & 21.7 \\ \text { 30-day } & 22.5 \\ \text { 60-day } & 24.6 \\ 90 \text {-day } & 26.8 \\ 20 \text {-day } & 28.0 \\ 83 \text {-day } & 34.5 \\ \text { 12-month } & 56.7\end{array}$

Duration of daily flow
Discharge (cfs)

Dates

Sept. 28, 1954

Sept. 30-0ct。 6, 1954

Sept. 28-oct。 7, 1954

Sept。24-Oct。13, 1954

Sept。15-0ct。14, 1954

Sept. 1-Oct. 30, 1954

Aug。3-0ct。31, 1954

Aug。1-Nov. 27, 1954

June 5-Dec。 4,1954

April 1954-March 1955

Storage required to maintain indicated flow

\begin{tabular}{cc}
$\begin{array}{c}\text { Percent time } \\
\text { indicated flow was } \\
\text { equaled or exceeded }\end{array}$ & $\begin{array}{c}\text { Discharge } \\
\text { (mgd) }\end{array}$ \\
\hline 20 & 70 \\
30 & 58 \\
50 & 39 \\
70 & 30 \\
80 & 27 \\
90 & 23 \\
95 & 18 \\
98 & 16 \\
99 & 15
\end{tabular}

\begin{tabular}{cc}
$\begin{array}{r}\text { Regulated flow } \\
\text { (mgd) }\end{array}$ & $\begin{array}{c}\text { Storage required } \\
\text { (million gallons) }\end{array}$ \\
\hline 15 & 26 \\
16 & 65 \\
18 & 180 \\
20 & 330 \\
23 & 620 \\
26 & 1,000 \\
30 & 1,600 \\
35 & 2,400 \\
40 & 3,200
\end{tabular}


Station No. 84

Location.-Lat $35^{\circ} 14^{\prime}$, long. $81^{\circ} 46^{\prime}$, a quarter of a mile downstrean from Cliffside Mills dam, at Cliffside, Rutherford County, and 1-1/2 miles upstream from mouth. Altitude of gage is $670 \mathrm{ft}$.

Drainage area.-211 sq mi.

Records available.-June 1925 to September 1955.

Flow summary.-1925-55:

Average discharge ( 30 years), 287 cfs.

Maximum di scharge, 15,000 cf's Aug。 14, 1940 (gage height, $17.93 \mathrm{ft}$ )。

Minimum discharge for indicated consecutive period:

Period Discharge (cfs) Dates

$\begin{array}{cc}\text { 1-day } & 6 \\ 7 \text {-day } & 39.1 \\ 10 \text {-day } & 40.8 \\ 20 \text {-day } & 44.3 \\ 30 \text {-day } & 51.7 \\ 60 \text {-day } & 56.0 \\ 90-\text { day } & 63.2 \\ 120 \text {-day } & 69.8 \\ 183 \text {-day } & 85.0 \\ 12 \text {-month } & 140\end{array}$

June 9, 1940

Oct. 28-Nove 3, 1926

Sept.29-0ct。7, 1926

Oct. 19-Nov。 7, 1926

Sept。10-0ct。9, 1954

Aug。30-0ct。28, 1954

Aug。 5-Nov。 2, 1954

July 23-Novo 19, 1954

June 6-Dec。 5,1954

July 1954-June 1955

Duration of daily flow

Storage required to maintain indicated flow

Percent time

indicated flow was Discharge

equaled or exceeded (mgd)

220

180

130

50

70

80

90

95

98

99

99.5

99.8

99.9
100

85

66

52

34

24

16

9.1

7.2
Regulated flow Storage required

\begin{tabular}{cc} 
(mgd) & (million gallons) \\
\hline 5.2 & 1.3 \\
10 & 6.4
\end{tabular}

25

22

30

40

42

50

60

300

900

70

80

100
1,800

3,200

4,600

9,200

Remarks.-Considerable diurnal fluctuation and some regulation caused by mills above station. 


\section{Broad River near Boiling Springs}

Station No. 85

Location.-Lat $35^{\circ} 12^{\prime} 35^{\prime \prime}$, long。 $81^{\circ} 41^{\prime} 50^{\prime \prime}$, half a mile upstream from Sandy Run Creek, 3 miles downstream from Second Broad River, and 3-1/2 miles southwest of Boiling Springs, Cleveland County. Datum of gage is 639.92 ft above mean sea level.

Drainage area. $-864 \mathrm{sq} \mathrm{mi}$.

Records available:-June 1925 to September 1955.

Flow summary.-1925-55:

Average discharge ( 30 years) 1,389 cfs.

Maximum discharge, 73,300 cfs Aug. 16, 1928 (gage height, $24.3 \mathrm{ft}$ )。

Minimum discharge for indicated consecutive period:

\begin{tabular}{ccl} 
Period & Discharge (cfs) & \multicolumn{1}{c}{ Dates } \\
1-day & 105 & Oct。10,1954 \\
7-day & 198 & Oct。 4-10, 1954 \\
10-day & 195 & Oct。1-10, 1954 \\
20-day & 218 & Sept。29-0ct. 18, 1954 \\
30-day & 233 & Sept。19-0ct。18, 1954 \\
60-day & 256 & Sept。9-Nov。7, 1954 \\
90-day & 295 & Aug。19-Nov。16, 1954 \\
120-day & 329 & July 23-Nov。19, 1954 \\
183-day & 397 & June 29-Dec。28, 1954 \\
12-month & 705 & April 1954-March 1955
\end{tabular}

Duration of daily flow

Storage required to maintain indicated flow

\begin{tabular}{cc}
\hline $\begin{array}{c}\text { Percent time } \\
\text { indicated flow was } \\
\text { equaled or exceeded }\end{array}$ & $\begin{array}{c}\text { Discharge } \\
\text { (mgd) }\end{array}$ \\
\hline 20 & 1,100 \\
30 & 920 \\
50 & 700 \\
70 & 530 \\
80 & 1,40 \\
90 & $31: 0$ \\
95 & 280 \\
98 & 220 \\
99 & 190 \\
99.5 & 170 \\
99.8 & 150 \\
99.9 & 120
\end{tabular}

\begin{tabular}{cc}
$\begin{array}{c}\text { Regulated flow } \\
\text { (mgd) }\end{array}$ & $\begin{array}{c}\text { Storage required } \\
\text { (million gallons) }\end{array}$ \\
\hline 74 & 5.8 \\
90 & 21 \\
130 & 66 \\
170 & 700 \\
200 & 2,000 \\
250 & 5,800 \\
300 & 11,000 \\
350 & 18,000 \\
400 & 26,000 \\
500 & 45,000
\end{tabular}

Remarks.-Considerable diurnal fluctuation and some regulation caused by powerplants above station. 


\section{First Broad River near Lawndale}

Station No。 86

Location. - Lat $35^{\circ} 22^{\prime} 50^{\prime \prime}$, long. $81^{\circ} 32^{\prime} 40^{\prime \prime}, 0.2$ mile upstream from Shoal Rock

Creek, $0.4 \mathrm{mile}$ downstream from highway bridge at Double Shools, and 2-1/2 miles southeast of Lawndele, Cleveland County。 Datum of gage is 735.94 above mean sea level, datum of 1929, supplementary adjustment of 1936.

\section{Drainage area.-198 sq $\mathrm{mi}$.}

Records available.-February 1940 to September 1955.

Flow summary. - 1940-55:

Average discharge ( 15 years), 260 cfs.

Maximum discharge, $32,500 \mathrm{cfs}$ Aug。 14, 1940 (gage height, $37.8 \mathrm{ft}$ ).

Flood of July 1916 reached a stage of $37.8 \mathrm{ft}$.

Minimum discharge for indicated consecutive period:

\begin{tabular}{rcl} 
Period & Discharge (cfs) & \multicolumn{1}{c}{ Dates } \\
& & \multicolumn{1}{c}{} \\
1-day & 23 & Sept。19, 1954 \\
7-day & 42.0 & Sept。13-19, 1954 \\
10-day & 40.8 & Sept。10-19, 1954 \\
20-day & 45.8 & Aug. 31-Sept。19, 1954 \\
30-day & 53.5 & Sept。23-0ct。22, 1954 \\
60-day & 63.1 & Sept。2-Oct。31, 1954 \\
90-day & 73.6 & Aug。1-Oct。29, 1954 \\
120-day & 65.5 & Jul。19-Nov。15, 1954 \\
183-day & 78.6 & Jun。28-Dec。27, 1954 \\
12-month & 185 & December 1950-November 1951
\end{tabular}

Duration of daily flow

Storage required to maintain indicated flow

\begin{tabular}{cc}
\hline $\begin{array}{c}\text { Percent time } \\
\text { indicated flow was } \\
\text { equaled or exceeded }\end{array}$ & $\begin{array}{c}\text { Discharge } \\
\text { (mgd) }\end{array}$ \\
\hline 20 & 190 \\
30 & 150 \\
50 & 120 \\
70 & 84 \\
80 & 73 \\
90 & 60 \\
95 & 50 \\
98 & 41 \\
99 & 34 \\
99.5 & 30 \\
99.8 & 25 \\
99.9 & 21
\end{tabular}

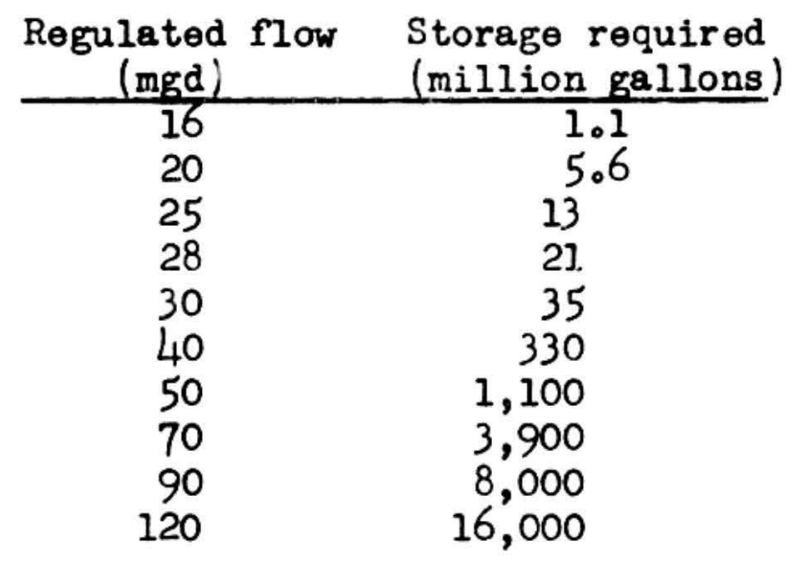

Remarks.-Considerable diurnal fluctuation and slight regulation at low flow caused by powerplants and mills above station. 


\section{South Fork New River near Jefferson}

Station No. 87

Location.-Lat $36^{\circ} 24^{\prime}$, long $81^{\circ} 25^{\prime}, 600 \mathrm{ft}$ upstrear from bridge on State Highways 16 and 88 , a quarter of a mile downstream from Bear Creek, and 4 miles southeast of Jefferson, Ashe County. Datum of gage is 2,657.04 ft above mean sea level, unadjusted.

Drainage area. -207 sq $\mathrm{mi}$.

Records available.--October 1924 to September 1926, July 1928 to September 1955.

Flow summary.-1924-26, 1928-55:

Average discharge $1925-26,1928-55$ (28 years), $410 \mathrm{cfs}$.

Maximum discharge, 52,800 cfs Aug。 1.4, 1940 (gage height, $22.50 \mathrm{ft}$ )。

Maximum stage known prior to 1924, 18.0 ft July 15, 1916 (discharge, $35,200 \mathrm{cfs})$.

Minimum discharge for indicated consecutive period:

\begin{tabular}{ccl} 
Period & Discharge (cfs) & \multicolumn{1}{c}{ Dates } \\
1-day & 65 & Sept。9, 1925 \\
7-day & 72.1 & Aug。21-27, 1925 \\
10-day & 76.5 & Aug。18-27, 1925 \\
20-day & 87.5 & Aug。21-Sept。9, 1925 \\
30-day & 90.3 & July 29-Aug.27, 1925 \\
60-day & 103 & July 29-Sept。26, 1925 \\
90-day & 105 & July 17-0ct。14, 1925 \\
120-day & 123 & June 27-Oct。24, 1925 \\
183-day & 145 & July 20-Nov。16, 1954 \\
12-month & 236 & April 1930-March 1931
\end{tabular}

Duration of daily flow

Storage required to maintain indicated flow

\begin{tabular}{cc}
\hline $\begin{array}{c}\text { Percent time } \\
\text { indicated flow was } \\
\text { equaled or exceeded }\end{array}$ & $\begin{array}{c}\text { Discharge } \\
\text { (mgd) }\end{array}$ \\
\hline 20 & 290 \\
30 & 2140 \\
50 & 190 \\
70 & 140 \\
80 & 110 \\
90 & 91 \\
95 & 78 \\
98 & 66 \\
99 & 61 \\
99.5 & 56 \\
99.8 & 51 \\
99.9 & 48
\end{tabular}

\begin{tabular}{cc}
$\begin{array}{c}\text { Regulated flow } \\
\text { (mgd) }\end{array}$ & $\begin{array}{c}\text { Storage required } \\
\text { (million gallons) }\end{array}$ \\
\hline 45 & 3.30 \\
52 & 40 \\
60 & 200 \\
70 & 600 \\
90 & 1,900 \\
110 & 4,000 \\
130 & 7,000 \\
150 & 10,000 \\
180 & 18,000
\end{tabular}




\section{North Fork New River at Crumpler}

Station No。 88

Location.-Lat $36^{\circ} 30^{\prime}$, long. $81^{\circ} 23^{\prime}$, a quarter of a mile downstream from bridge on State Highway 16 at Crumpler, Ashe County, about 6 miles upstream from mouth, and 7-1/2 miles northeast of Jefferson. Datum of gage is 2,518.81 ft above mean sea level, unadjusted.

Drainage area. -277 sq $\mathrm{mi}$.

Records available.-August 1900 to December 1901 (fragmentary), August 1908 to September 1916, and July 1928 to September 1955.

Flow summary.-1908-16, 1928-55:

Average discharge ( 35 years), 467 cfs.

Maximum discharge, $79,400 \mathrm{cfs}$ Aug。 14, 1940 (gage height, $23.0 \mathrm{ft}$ ).

Minimum discharge for indicated consecutive period:

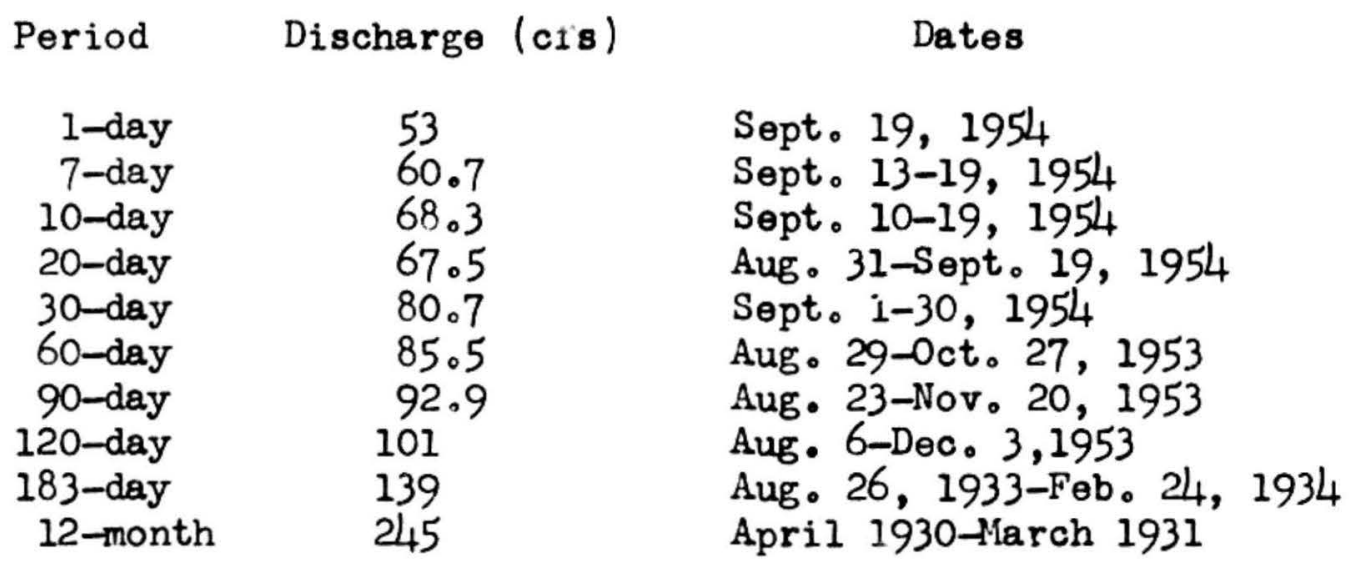

Duration of daily flow

Storage required to maintain indicated flow

\begin{tabular}{cc}
\hline $\begin{array}{c}\text { Percent time } \\
\text { indicated flow was } \\
\text { equaled or exceeded }\end{array}$ & $\begin{array}{c}\text { Discharge } \\
\text { (mgd) }\end{array}$ \\
\hline 20 & 390 \\
30 & 310 \\
50 & 220 \\
70 & 150 \\
30 & 120 \\
90 & 87 \\
95 & 71 \\
98 & 60 \\
99 & 53 \\
99.5 & 48
\end{tabular}

\begin{tabular}{cc}
$\begin{array}{c}\text { Regulated flow } \\
\text { (mgd) }\end{array}$ & $\begin{array}{c}\text { Storage required } \\
\text { (million gallons) }\end{array}$ \\
\hline 36 & 2.0 \\
40 & 10 \\
45 & 50 \\
50 & 160 \\
60 & 520 \\
70 & 1,200 \\
90 & 3,000 \\
110 & 5,400 \\
135 & 9,500 \\
160 & 13,000
\end{tabular}

Remarks.- Some diurnal fluctuation at low flow caused by powerplant above station. 